\title{
LYAPUNOV SPECTRUM OF BALL QUOTIENTS WITH APPLICATIONS TO COMMENSURABILITY QUESTIONS
}

\author{
ANDRÉ KAPPES AND MARTIN MÖLLER
}

\begin{abstract}
We determine the Lyapunov spectrum of ball quotients arising from cyclic coverings. The computations are performed by rewriting the sum of Lyapunov exponents as ratios of intersection numbers and by the analysis of the period map near boundary divisors.

As a corollary, we complete the classification of commensurability classes of all presently known non-arithmetic ball quotients.
\end{abstract}

\section{Contents}

1. Introduction

2. Background

3. Lyapunov exponents of ball quotients: Generalities

4. Invariants of modular embeddings and commensurability invariants

5. Arithmeticity

6. Cyclic covers

7. Lyapunov exponents of ball quotients constructed via cyclic coverings

8. Orbifold Euler numbers and log-ball quotients

References

\section{INTRODUCTION}

This paper is intended to contribute to the problem of classifying commensurability classes of non-arithmetic ball quotients with a technique that has been useful to understand the Teichmüller geodesic flow, the calculation of the Lyapunov spectrum.

By Margulis' arithmeticity theorem ( Mar91]) non-arithmetic lattices only exist in Lie groups of rank one. While there are irreducible non-arithmetic lattices in the isometry group of real hyperbolic space of dimension $n$ for any $n$, the construction of non-arithmetic lattices in the isometry group of complex hyperbolic $n$-space $\mathrm{PU}(1, n)$ is a hard open problem if $n>1$.

Up to commensurability, al[ the presently known non-arithmetic ball quotients arise from cyclic coverings of the projective line, an investigation started by [DM86]

Date: November 2, 2018.

The authors are partially supported by the ERC-StG 257137 .

${ }^{1}$ After the completion of this work, finitely many new non-arithmetic ball quotients not commensurable to the Deligne-Mostow examples have been found by Deraux, Parker and Paupert DPP14, see also DPP11] 
and completed by Mos86 and Thu98. Other constructions, earlier and shortly after the work of Deligne, Mostow and Thurston, turned out to be commensurable to these ball quotients. In fact, the book DM93 gathers a lot of techniques to detect commensurabilities between lattices.

To detect non-commensurability, the only technique appearing in the literature seems to be the trace field and (non-)compactness. As a consequence, Pau10, Remark 5.1] asks whether among the 15 cyclic covering examples, there are 7, 8 or 9 commensurability classes. As one result of our methods we show that there are in fact 9 classes. The first new commensurability invariant that we propose is the set of Lyapunov exponents of a variation of Hodge structures associated with ball quotients arising from cyclic coverings. We first give some background on these notions and then explain that they fit into a larger class of commensurability invariants.

Cyclic coverings. The ball quotients of Deligne and Mostow stem from families of algebraic curves that are cyclic coverings of $\mathbb{P}^{1}$ branched at $N$ points. For a fixed degree $d$ and a ramification datum $\left(a_{1}, \ldots, a_{N}\right)$, one considers the algebraic curve

$$
y^{d}=\prod_{i=1}^{N}\left(x-x_{i}\right)^{a_{i}}, \quad\left(x_{1}, \ldots, x_{N}\right) \in \mathcal{M}_{0, N}
$$

Moving the branch points in $\mathcal{M}_{0, N}$ yields a family of curves. The first cohomology groups of the fibers of this family form a local system (or flat vector bundle) on $\mathcal{M}_{0, N}$ and the (1,0)-subspaces of the Hodge decomposition yield a holomorphically varying subbundle. This is the prototypical example of a variation of Hodge structures of weight 1 (VHS) to have in mind for the theorem below (see Section 2.2 for a definition of VHS).

Using the action of the Galois group $\mathbb{Z} /(d)$ of the covering, one can decompose the cohomology into eigenspaces; these form themselves variations of Hodge structures defined over some cyclotomic field, and the natural polarization on the fibers sometimes is a hermitian form of signature $(1, n)$, where $n=N-3$. In this case, the period map, which records the position of the $(1,0)$-subspace in the cohomology, is a holomorphic map from the universal cover of $\mathcal{M}_{0, N}$ to a ball $\mathbb{B}^{n}$, which is the parameter space of Hodge structures of this type. The fundamental group of $\mathcal{M}_{0, N}$ in turn acts on a fiber of the local system by parallel transport of flat sections; since it preserves the hermitian form, this yields a representation into $\mathrm{PU}(1, n)$, and the period map is equivariant for the two actions of the fundamental group. The main achievement of [DM86] is to define a suitable compactification of $\mathcal{M}_{0, N}$ and to determine for which parameters $\left(d ; a_{i}\right)$ and which eigenspace one can extend the period map to yield an isomorphism with the complex ball. In this case, the representation of the fundamental group gives a lattice in $\mathrm{PU}(1, n)$, and the VHS is called uniformizing.

Lyapunov exponents. The Lyapunov exponents of a VHS of rank $k$ are $2 k$ real numbers $\lambda_{i}$ that group symmetrically around 0 and measure, roughly speaking, the logarithmic growth rate of cohomology classes under parallel transport along the geodesic flow of the ball quotient. By Oseledets' theorem such Lyapunov exponents can be associated to any ergodic cocycle under a weak integrability hypothesis which holds for variations of Hodge structures, see Lemma 2.7. 
Lyapunov exponents for VHS have first been investigated for the space $\Omega \mathcal{M}_{g}$ of flat surfaces, i.e. pairs $(X, \omega)$ of a compact Riemann surface together with a nonzero holomorphic one-form. This space admits a natural action of $\mathrm{SL}_{2}(\mathbb{R})$, and the action of the diagonal subgroup is the Teichmüller geodesic flow, see [Zor06] for a survey. In this situation Lyapunov exponents have been determined e.g. for families of cyclic coverings branched over 4 points in [BM10] and [EKZ11]. The simplicity of the spectrum (but no precise values) are known for a generic flat surface $(X, \omega)$ of genus at least two. A fundamental observation of Kontsevich and Zorich (see Kon97 and also EKZ14) relates the sum of Lyapunov exponents to the degree of the Hodge bundle (or a summand, if the Hodge structure splits) and this degree can actually be calculated.

For a comparison with our Theorem 1.1 we state Kontsevich's formula for the sum of Lyapunov exponents in the case of a family of curves $f: \mathcal{X} \rightarrow C$ over a compact hyperbolic curve $C=\mathbb{H} / \Gamma$. The VHS in question is the relative cohomology $R^{1} f_{*} \mathbb{C}$ together with the subbundle $f_{*} \omega_{\mathcal{X} / C}$ of relative 1 -forms pushed forward to $C$. In this case,

$$
\sum_{i=1}^{g} \lambda_{i}=\frac{2 c_{1}\left(f_{*} \omega_{\mathcal{X} / C}\right)}{c_{1}\left(\omega_{C}\right)}
$$

If $C$ is not compact as in the case of Teichmüller curves, one has to take the Deligne extension of $f_{*} \omega_{\mathcal{X} / C}$ and the canonical bundle $\omega_{\bar{C}}$ of the completion $\bar{C}$ instead.

Kontsevich's observation can be extended to ball quotients, since they are KählerEinstein manifolds as in the one-dimensional case. More precisely, we have the following result, which we state for abstract variations of Hodge structures.

Theorem 1.1. Suppose that $\mathbb{V}$ is a real polarized variation of Hodge structures of weight 1 over a ball quotient $B=\mathbb{B}^{n} / \Gamma$ of constant curvature -4 , where $\Gamma$ is a torsionfree lattice in $\mathrm{PU}(1, n)$. Let $\bar{B}$ be a smooth compactification of $B$ with normal crossing boundary divisor $\Delta$, and assume that the local monodromy of $\mathbb{V}$ about $\Delta$ is unipotent. Then the Lyapunov spectrum of $\mathbb{V}$ has the following properties.

i) Normalization. If $\mathbb{V}_{\mathbb{C}}=\mathbb{V} \otimes_{\mathbb{R}} \mathbb{C}$ has an irreducible summand which is uniformizing, then the top Lyapunov exponent is one.

ii) Duplication. If an $\mathbb{R}$-irreducible direct summand $\mathbb{W}$ of $\mathbb{V}$ is reducible over $\mathbb{C}$, then each Lyapunov exponent of $\mathbb{W}$ has even multiplicity.

iii) Zero exponents for non-real factors. If an irreducible summand $\mathbb{W}_{\mathbb{C}}$ of $\mathbb{V}_{\mathbb{C}}$ has signature $(p, q)$, then at least $2|p-q|$ of the Lyapunov exponents corresponding to $\mathbb{W}_{\mathbb{C}}$ are zero.

iv) Partial sums are intersection numbers. Let $\mathbb{W}$ be a direct summand of rank $2 k$ in the decomposition of $\mathbb{V}$. Then the positive Lyapunov exponents $\lambda_{1}, \ldots, \lambda_{k}$ of $\mathbb{W}$ satisfy

$$
\lambda_{1}+\cdots+\lambda_{k}=\frac{(n+1) \mathrm{c}_{1}\left(\mathcal{E}^{1,0}\right) \cdot \mathrm{c}_{1}\left(\omega_{\bar{B}}\right)^{n-1}}{\mathrm{c}_{1}\left(\omega_{\bar{B}}\right)^{n}},
$$

where $\omega_{\bar{B}}=\bigwedge^{n} \Omega \frac{1}{B}(\log \Delta)$, and $\mathcal{E}^{1,0}$ is the Deligne extension of $\mathcal{W}^{1,0} \subset$ $\mathbb{W} \otimes \mathcal{O}_{B}$

Commensurability invariants. Due to their construction via families of curves, all the ball quotients arising from cyclic coverings come with a $\mathbb{Q}$-VHS that contains a uniformizing sub-VHS. The Galois conjugates of this sub-VHS constitute 
the primitive part that we associate to such a ball quotient (see Definition 2.4 for the precise statement). The local system of the primitive part only depends on the lattice as it corresponds to the sum of the representations $\Gamma \rightarrow \Gamma^{\sigma}$ given by Galois conjugation. On the other hand, the Hodge decomposition is a priori not an intrinsic datum of the lattice. However, it is unique if it exists. This is shown in Theorem 4.3 using the notion of modular embeddings. Informally, a modular embedding is the collection of the equivariant period maps associated with the different representations $\Gamma \rightarrow \Gamma^{\sigma}$. Using this terminology, we show in Section 4

Theorem 1.2 (see Theorem 4.8). The Lyapunov spectrum of the primitive part is a commensurability invariant among lattices in $\mathrm{PU}(1, n)$ that admit a modular embedding.

The right hand side of (2) can also be generalized to give a new class of commensurability invariants. In general, for lattices as in Theorem 1.2 the ratios of the form

$$
\frac{\mathrm{c}_{1}\left(\mathcal{E}^{1,0}\right)^{a} \cdot \mathrm{c}_{1}\left(\omega_{\bar{B}}\right)^{b}}{\mathrm{c}_{1}\left(\omega_{\bar{B}}\right)^{n}}
$$

for all $(a, b)$ with $a+b=n$ are natural invariants of the commensurability class of $\Gamma$. Here the $\mathcal{E}^{1,0}$ correspond to irreducible summands in the primitive part of the VHS associated with $\Gamma$. We refer to Corollary 4.5 for the precise statement.

The second part of this paper shows that for all presently known commensurability classes of ball quotients all individual Lyapunov exponents in the primitive part can be calculated using Theorem 1.1 and local computations.

First of all, arithmetic lattices are of little interest in this context. As a consequence of Theorem 1.1 we show in Proposition 5.1 that for an arithmetic lattice the primitive Lyapunov spectrum is maximally degenerate, i.e. it consists of $\{+1,0,-1\}$ only and the number of +1 is determined by the signature of the Hodge inner product.

The non-arithmetic examples are much more interesting. We calculate the individual Lyapunov exponents of the primitive part for all known non-arithmetic examples in Theorem 7.1. We state a particularly interesting case here.

Theorem 1.3. Let $f: \mathcal{X} \rightarrow B_{0}=\left(\mathbb{P}^{1}\right)^{5} \backslash$ (diagonals) be the family of cyclic coverings of $\mathbb{P}^{1}$ given by

$$
y^{12}=\left(x-x_{1}\right)^{3}\left(x-x_{2}\right)^{3}\left(x-x_{3}\right)^{5}\left(x-x_{4}\right)^{6}\left(x-x_{5}\right)^{7},
$$

respectively by

$$
y^{12}=\left(x-x_{1}\right)^{4}\left(x-x_{2}\right)^{4}\left(x-x_{3}\right)^{4}\left(x-x_{4}\right)^{5}\left(x-x_{5}\right)^{7} .
$$

Let $\mathbb{L}$ be the uniformizing direct summand of $R^{1} f_{*} \mathbb{C}$ that when extended to a suitable compactification $B$ of $B_{0}$ exhibits $B$ as a non-compact orbifold ball quotient. Then the Lyapunov exponents of the primitive part $\mathbb{P}$ are given by

$$
1, \frac{5}{17}, 0,-\frac{5}{17},-1, \quad \text { respectively } \quad 1, \frac{7}{22}, 0,-\frac{7}{22},-1,
$$

where $\mathbb{P}$ in this case is the $\mathbb{C}$-subvariation associated with the direct sum of the Galois conjugates of the $\mathbb{Q}\left(\zeta_{12}\right)$-form of $\mathbb{L}$.

As a corollary to this theorem we obtain that the two associated lattices are not commensurable to each other. The full commensurability statement mentioned at the beginning is given in Corollary 7.2 
In order to prove Theorem [7.1 we reduce the calculation of $c_{1}\left(\mathcal{E}^{1,0}\right)$ to the computation of local invariants near the 'boundary divisors', where two of the branch points collapse. It turns out that it suffices to compute the cokernel of Kodaira-Spencer maps near these boundary divisors and this will be done by a local analysis of hypergeometric integrals. As final piece of information we need the intersection rings and Chern classes of the tangent bundle of the moduli spaces of weighted stable curves (or their quotients by finite groups in the case (इINT)).

Naturally, one would also like to calculate the Lyapunov exponents of the nonprimitive part of the VHS for a cyclic covering. However, one runs into the following problem.

Remark 1.4. The problem of calculating the Lyapunov exponents of the whole variation of Hodge structures $R^{1} f_{*} \mathbb{C}$ of a family of cyclic coverings branched over $N \geq 5$ points is not well-defined in any of the non-arithmetic cases, since there always exists a direct summand that does not extend to the ball, i.e. whose period map is not defined on $\mathbb{B}^{n}$ (see Example 6.5). For a discussion of the case $N=4$, we refer to [BM10, Wri12, and [EKZ11].

Orbifold Euler numbers. After a first version of this paper was circulated, McMullen pointed out to us that one can define another invariant for non-arithmetic ball quotients coming from cyclic coverings, the relative orbifold Euler numbers. For each Galois conjugate $\Gamma^{\sigma}$ of $\Gamma$, there is a hyperbolic cone manifold $B^{\sigma}$ (with $B^{\text {id }}=\mathbb{B}^{n} / \Gamma$ being the usual orbifold ball quotient). The relative Euler numbers are then defined as the collection $\mathrm{e}^{\text {orb }}\left(B^{\sigma}\right) / \mathrm{e}^{\text {orb }}\left(B^{\text {id }}\right)$. The precise definition, entirely combinatorial, is given in Section 8 and an explicit formula is stated in Proposition 8.3 McMullen's observation was that the two sets of invariants, positive Lyapunov exponents and relative orbifold Euler numbers, agree for one-dimensional ball quotients, but differ in dimension two 2 . Our next result, a simplified restatement of Corollary 8.2 gives the explanation.

Theorem 1.5. Let $\mathbb{L}^{\sigma}$ be a Galois conjugate of the uniformizing VHS $\mathbb{L}$ of a family of cyclic coverings. Suppose $n=2$ and that the polarization of $\mathbb{L}^{\sigma}$ has signature $(1,2)$. Then

$$
\frac{\mathrm{e}^{\mathrm{orb}}\left(B^{\sigma}\right)}{\mathrm{e}^{\mathrm{orb}}\left(B^{\mathrm{id}}\right)}=\frac{\left(3 \cdot \mathrm{c}_{1}\left(\mathcal{E}^{1,0}\right)\right)^{2}}{\mathrm{c}_{1}\left(\omega_{\bar{B}}\right)^{2}}
$$

For the families of curves given by (4) and (5) the set of relative orbifold Euler numbers is

$$
1, \frac{1}{17}, \quad \text { respectively } \quad 1, \frac{1}{22}
$$

Said differently, the sum of Lyapunov exponents corresponds to the case $(1, n-1)$ of the invariants in (3) while the orbifold Euler characteristics correspond to the case $(2,0)$.

To prove Theorem 1.5 we show in Theorem 8.1 that $B^{\sigma}$ is a $\log$ ball quotient, i.e. for an appropriate choice of weights on boundary divisors this log manifolds attains the upper bound in Langer's logarithmic version of the Bogomolov-Miyaoka-Yau inequality ( Lan03]).

\footnotetext{
${ }^{2}$ The values that McMullen calculated (meanwhile also available in the preprint 'The GaussBonnet theorem for cone manifolds and volumes of moduli spaces' on his web page) match with our Corollary 8.2 They are included in the table in Theorem 7.1
} 
Structure of the paper. The plan of the paper is as follows. In Section 2 we provide background information about ball quotients, Lyapunov exponents and variations of Hodge structures. Section 3 contains the proof of Theorem 1.1. Section 4 contains the definition of modular embeddings for ball quotients and the proof of commensurability invariance of the primitive Lyapunov spectrum. The arithmetic case is quickly discussed in Section 5 . The basic facts about cyclic covers and those cases that give rise to (non-arithmetic) ball quotients are recalled in Section 6. This section also contains the description of intersection rings of these ball quotients.

Acknowledgments. The authors thank Jürgen Wolfart for useful comments on modular embeddings, Simion Filip for useful comments on the proof of Lemma 2.7, Curt McMullen for asking about the relation between Lyapunov exponents and orbifold Euler numbers and the referees for their valuable suggestions to improve the exposition of the paper.

\section{BACKGROUND}

2.1. The complex ball and its Kähler structure. We first collect some wellknown facts about the Kähler structure of ball quotients. We carefully carry along the dependence on the curvature in order to have a consistent normalization in Theorem $1.1 \mathrm{i})$. Let $\mathbb{C}^{1, n}$ be $\mathbb{C}^{n+1}$ equipped with the following hermitian pairing. For $\underline{W}=\left(W_{0}, W\right)$ and $\underline{Z}=\left(Z_{0}, Z\right) \in \mathbb{C}^{n+1}$ we let

$$
\langle\underline{W}, \underline{Z}\rangle_{1, n}=W_{0} \overline{Z_{0}}-(W, Z),
$$

where $(W, Z)=\sum_{i=1}^{n} W_{i} \overline{Z_{i}}$. Then $\mathbb{H}_{\mathbb{C}}^{n}$ is the space of lines where the hermitian pairing is positive definite. We may identify $\mathbb{H}_{\mathbb{C}}^{n}$ with

$$
\mathbb{B}^{n}=\left\{z=\left(z_{1}, \ldots, z_{n}\right): \sum\left|z_{i}\right|^{2}<1\right\}
$$

via the map $f: \mathbb{B}^{n} \rightarrow \mathbb{P}\left(\mathbb{C}^{1, n}\right), z \mapsto(1: z)$.

$\mathbb{B}^{n}$ is naturally endowed with a Kähler structure, whose Kähler form in these coordinates is given by

$$
\begin{aligned}
& \omega_{\text {hyp }}=2 \kappa i \partial \bar{\partial} \log \left(\frac{1}{2}\langle f(z), f(z)\rangle_{1, n}\right) \\
& =\frac{2 \kappa i}{(1-(z, z))^{2}}\left\{\left(\sum_{j=1}^{n} \bar{z}_{j} d z_{j}\right) \wedge\left(\sum_{k=1}^{n} z_{k} d \bar{z}_{k}\right)+(1-(z, z)) \sum_{j=1}^{n} d z_{j} \wedge d \bar{z}_{j}\right\}
\end{aligned}
$$

with $\kappa>0$ Gol99. Its holomorphic sectional curvature is $\frac{-2}{\kappa}$. Setting $\kappa=\frac{1}{2}$, we obtain the metric of Theorem 1.1.

The corresponding Riemannian metric is given by

$$
g_{\mathrm{hyp}}=\frac{2 \kappa}{(1-(z, z))^{2}}\left\{\left(\sum_{j=1}^{n} \bar{z}_{j} d z_{j}\right)\left(\sum_{k=1}^{n} z_{k} d \bar{z}_{k}\right)+(1-(z, z)) \sum_{j=1}^{n} d z_{j} d \bar{z}_{j}\right\} .
$$

The volume form is

$$
\mathrm{dvol}_{\mathbb{B}^{n}}=\frac{1}{n !} \omega_{\mathrm{hyp}}^{n}=\frac{(2 \kappa i)^{n}}{(1-(z, z))^{n+1}} d z_{1} \wedge d \bar{z}_{1} \wedge \cdots \wedge d z_{n} \wedge d \bar{z}_{n} .
$$


2.1.1. The Laplacian on the complex ball. Let $\Delta_{\text {hyp }}$ be the Laplacian for the Riemannian metric on $\mathbb{B}^{n}$. It is given by

$$
\Delta_{\text {hyp }}=\frac{1}{\kappa}(1-(z, z))\left(\sum_{i=1}^{n} \frac{\partial^{2}}{\partial z_{i} \partial \bar{z}_{i}}-\sum_{i, j=1}^{n} z_{i} \bar{z}_{j} \frac{\partial^{2}}{\partial z_{i} \partial \bar{z}_{j}}\right) .
$$

Lemma 2.1. If $F$ is a smooth function on the ball, we have

$$
\Delta_{\text {hyp }}(F) \cdot \operatorname{dvol}_{\mathbb{B}^{n}}=\frac{2 i}{(n-1) !} \cdot \partial \bar{\partial} F \wedge \omega_{\text {hyp }}^{n-1}
$$

Proof. Since $\mathbb{B}^{n}$ is Kähler, there is a coordinate system $w_{1}, \ldots, w_{n}$ centered at $x \in \mathbb{B}^{n}$ such that in the fiber over $x$

$$
\omega_{x}=2 \kappa i \sum_{j=1}^{n} d w_{j, x} \wedge d \bar{w}_{j, x}, \quad \text { and } \quad \Delta_{\mathrm{hyp}}(F)_{x}=\left.\frac{1}{\kappa} \sum_{i=1}^{n} \frac{\partial^{2} F}{\partial w_{i} \partial \bar{w}_{i}}\right|_{x} .
$$

Therefore, it suffices to prove (9) for this particular coordinate system, which is a straightforward calculation.

2.1.2. Geodesic polar coordinates. We use the parametrization of $\mathbb{B}^{n}$ by geodesic polar coordinates centered at $x \in \mathbb{B}^{n}$. Let $\theta=\left(\theta_{1}, \ldots, \theta_{2 n-1}\right)$ be a local parametrization of $T_{x}^{1} \mathbb{B}^{n} \subset T_{x} \mathbb{B}^{n}$. Then the map

$$
\exp _{x}: T_{x} \mathbb{B}^{n} \rightarrow \mathbb{B}^{n}, \quad(t, \theta) \mapsto \exp _{x}\left(t \theta_{1}, \ldots, t \theta_{2 n-1}\right)
$$

parametrizes $\mathbb{B}^{n}$ by geodesic polar coordinates.

The volume form on $\mathbb{B}^{n}$ written in geodesic polar coordinates is

$$
\operatorname{dvol}_{\mathbb{B}^{n}}=\frac{(4 \kappa)^{n}}{2 \sqrt{2 \kappa}} \sinh ^{2 n-2}\left(\frac{t}{\sqrt{2 \kappa}}\right) \sinh \left(\frac{2 t}{\sqrt{2 \kappa}}\right) \mathrm{d} t \mathrm{~d} \sigma,
$$

where $\mathrm{d} \sigma$ denotes the volume form on $T_{x}^{1} \mathbb{B}^{n}$. This follows from (7) and the $\mathrm{PU}(1, n)$ invariance, by which we can reduce the computation to $x=0$. Further, we use the equality

$$
\left(\frac{i}{2}\right)^{n} \mathrm{~d} z_{1} \wedge \mathrm{d} \bar{z}_{1} \wedge \cdots \wedge \mathrm{d} z_{n} \wedge \mathrm{d} \bar{z}_{n}=\mathrm{d} x_{1} \wedge \mathrm{d} y_{1} \wedge \cdots \wedge \mathrm{d} x_{n} \wedge \mathrm{d} y_{n}
$$

and

$$
\mathrm{d} x_{1} \wedge \mathrm{d} y_{1} \wedge \cdots \wedge \mathrm{d} x_{n} \wedge \mathrm{d} y_{n}=r^{2 n-1} \mathrm{~d} r \mathrm{~d} \sigma .
$$

Moreover, the Euclidean radius $r$ is related to the hyperbolic radius $t$ by $r=$ $\tanh (t /(\sqrt{2 \kappa}))$. Hence the formula follows using $\cosh (x) \sinh (x)=\frac{1}{2} \sinh (2 x)$.

Moreover, we need the volume of $\mathbb{B}_{t}^{n}=\mathbb{B}_{t}^{n}(x)=\left\{z \in \mathbb{B}^{n} \mid d_{\text {hyp }}(x, z)<t\right\}$. It is given by

$$
\operatorname{vol}\left(\mathbb{B}_{t}^{n}\right)=\int_{0}^{t} \int_{T_{x}^{1} \mathbb{B}^{n}} \operatorname{dvol}_{\mathbb{B}^{n}}=\frac{(4 \kappa)^{n} \sigma_{2 n-1}}{2 n} \sinh ^{2 n}\left(\frac{t}{\sqrt{2 \kappa}}\right) .
$$

Here $\sigma_{2 n-1}$ is the Euclidean volume of $T_{x}^{1} \mathbb{B}^{n}$.

Finally, by [Hel94 the Laplacian on $\mathbb{B}^{n}$ written in geodesic polar coordinates centered at 0 is

$$
\Delta_{\text {hyp }}=\frac{\partial^{2}}{\partial t^{2}}+\frac{1}{A(t)} \frac{\mathrm{d} A}{\mathrm{~d} t} \frac{\partial}{\partial t}+\Delta_{S_{t}(0)}
$$

where $A(t)$ is the area of the sphere $S_{t}(0) \subset \mathbb{B}^{n}$ of radius $t$ centered at 0 . Here $\Delta_{S_{t}(0)}$ is the Laplacian on $S_{t}(0)$ for the Riemannian metric induced from the one on $\mathbb{B}^{n}$. 
We have

$$
A(t)=\frac{\mathrm{d}}{\mathrm{d} t} \operatorname{vol}\left(\mathbb{B}_{t}^{n}\right)=\frac{(4 \kappa)^{n} \sigma_{2 n-1}}{2 \sqrt{2 \kappa}} \sinh ^{2 n-2}\left(\frac{t}{\sqrt{2 \kappa}}\right) \sinh \left(\frac{2 t}{\sqrt{2 \kappa}}\right)
$$

Therefore the factor in front of $\partial / \partial t$ in (10) is

$$
\begin{aligned}
A(t)^{-1} A^{\prime}(t) & =A(t)^{-1} \cdot(4 \kappa)^{n-1} \sigma_{2 n-1} 2 \sinh ^{2 n-2}\left(\frac{t}{\sqrt{2 \kappa}}\right)\left(2 n \cosh ^{2}\left(\frac{t}{\sqrt{2 \kappa}}\right)-1\right) \\
& =\frac{\sqrt{2 \kappa}}{\kappa}\left(n \operatorname{coth}\left(2 \frac{t}{\sqrt{2 \kappa}}\right)+\frac{n-1}{\sinh \left(2 \frac{t}{\sqrt{2 \kappa}}\right)}\right)
\end{aligned}
$$

The following version of Green's formula was used in For02 for the hyperbolic plane. It carries over to higher dimension.

Lemma 2.2. Let $x \in \mathbb{B}^{n}$, let $\Lambda: \mathbb{B}^{n} \rightarrow \mathbb{R}$ be a smooth function, and let $L$ be $a$ smooth solution to the Poisson equation $\Delta L=\Lambda$. Then

$$
\frac{1}{\sigma_{2 n-1}} \frac{\partial}{\partial t} \int_{T_{x}^{1} \mathbb{B}^{n}} L\left(\exp _{x}(t, \theta)\right) d \sigma=\frac{\sqrt{2 \kappa}}{2 n} \tanh \left(\frac{t}{\sqrt{2 \kappa}}\right) \cdot \frac{1}{\operatorname{vol}\left(\mathbb{B}_{t}^{n}\right)} \int_{\mathbb{B}_{t}^{n}(x)} \Lambda \operatorname{dvol}_{\mathbb{B}^{n}}
$$

Proof of Lemma 2.2. By homogeneity, we can assume $x=0$. Proceeding in exactly the same way as in For02, we set

$L_{r}(t)=\frac{1}{\sigma_{2 n-1}} \int_{T_{0}^{1} \mathbb{B}^{n}} L\left(\exp _{0}(t, \theta)\right) \mathrm{d} \sigma \quad$ and $\quad \Lambda_{r}(t)=\frac{1}{\sigma_{2 n-1}} \int_{T_{0}^{1} \mathbb{B}^{n}} \Lambda\left(\exp _{0}(t, \theta)\right) \mathrm{d} \sigma$

Then since $\Delta_{S_{t}(0)} L_{r}=0$ as it involves only partial derivatives in $\theta$, we have

$$
\Delta_{\mathrm{hyp}} L_{r}(t)=\frac{\partial^{2}}{\partial t^{2}} L_{r}(t)+f(t) \frac{\partial}{\partial t} L_{r}(t)=\Lambda_{r}(t)
$$

Thus $u(t)=\frac{\partial}{\partial t} L_{r}(t)$ satisfies a first-order ODE, whose solution is

$$
u(t)=\frac{1}{\sinh ^{2 n-2}\left(\frac{t}{\sqrt{2 \kappa}}\right) \sinh \left(\frac{2 t}{\sqrt{2 \kappa}}\right)} \int_{0}^{t} \Lambda_{r}(\tau) \sinh ^{2 n-2}\left(\frac{\tau}{\sqrt{2 \kappa}}\right) \sinh \left(\frac{2 \tau}{\sqrt{2 \kappa}}\right) \mathrm{d} \tau
$$

Therefore,

$$
\begin{aligned}
\frac{1}{\sigma_{2 n-1}} u(t)= & \frac{(4 \kappa)^{n}}{2 n} \sinh ^{2 n}\left(\frac{t}{\sqrt{2 \kappa}}\right) \cdot \frac{1}{\operatorname{vol}\left(\mathbb{B}_{t}^{n}\right)} u(t) \\
= & \frac{\sqrt{2 \kappa}}{n} \cdot \frac{\sinh ^{2}\left(\frac{t}{\sqrt{2 \kappa}}\right)}{\sinh \left(\frac{2 t}{\sqrt{2 \kappa}}\right)} \cdot \frac{1}{\operatorname{vol}\left(\mathbb{B}_{t}^{n}\right)} \cdot \\
& \cdot \int_{0}^{t} \int_{T_{0}^{1} \mathbb{B}^{n}} \Lambda\left(\exp _{0}(\tau, \theta)\right) \frac{(4 \kappa)^{n}}{2 \sqrt{2 \kappa}} \sinh ^{2 n-2}\left(\frac{\tau}{\sqrt{2 \kappa}}\right) \sinh \left(\frac{2 \tau}{\sqrt{2 \kappa}}\right) \mathrm{d} \tau \mathrm{d} \sigma \\
= & \frac{\sqrt{2 \kappa}}{2 n} \tanh \left(\frac{t}{\sqrt{2 \kappa}}\right) \frac{1}{\operatorname{vol}\left(\mathbb{B}_{t}^{n}\right)} \int_{\mathbb{B}_{t}^{n}} \Lambda \operatorname{dvol}_{\mathbb{B}^{n}}
\end{aligned}
$$


2.2. Weight one variations of Hodge structures (VHS). The first cohomology of a complex algebraic curve $X$ naturally carries a Hodge decomposition $H^{1}(X, \mathbb{C})=H^{1,0} \oplus H^{0,1}$ into the direct sum of classes of holomorphic and antiholomorphic forms. The generalization of the Hodge decomposition to families of curves or more generally to families of compact Kähler manifolds is abstracted by the notion of a variation of Hodge structres. Below, we collect the basic definitions needed. For simplicity, we state everything for weight one only. A general reference for this section is [CMSP03].

Let $K \subset \mathbb{R}$ be a field, and let $W$ be a $K$-vector space. By a $K$-Hodge structure (of weight one) on $W$, we understand a decomposition $W \otimes_{K} \mathbb{C}=W^{1,0} \oplus W^{0,1}$, such that $\overline{W^{1,0}}=W^{0,1}$. We extend this notion to $K=\mathbb{C}$ by dropping the condition that $\overline{W^{1,0}}=W^{0,1}$.

Let $B$ be a base manifold which is the complement in the projective variety $Y$ of a normal crossing divisor $\Delta$. A (weight one) $K$-variation of Hodge structures over $B$ consists of a $K$-local system $\mathbb{W}$ on $B$ and a $C^{\infty}$-decomposition

$$
\mathbb{W} \otimes_{K} \mathcal{O}_{B}=\mathcal{W}^{1,0} \oplus \mathcal{W}^{0,1}
$$

into a holomorphic (resp. antiholomorphic) subbundle $\mathcal{W}^{1,0}$ (resp. $\mathcal{W}^{0,1}$ ) that induces fiber by fiber a Hodge structure of weight 1 . Note that for higher weight, there is an additional transversality condition, which is vacuous for weight one.

A $\mathbb{C}$-VHS $\mathbb{W}$ is called polarized, if there is a locally constant hermitian form $\psi$ on $\mathbb{W}$, for which the decomposition $\mathcal{W}^{1,0} \oplus \mathcal{W}^{0,1}$ is orthogonal, and which is positive definite on $\mathcal{W}^{1,0}$ and negative definite on $\mathcal{W}^{0,1}$. Consequently, its signature is $\left(\mathrm{rk} \mathcal{W}^{1,0}, \mathrm{rk} \mathcal{W}^{0,1}\right)$. This indefinite hermitian form will be referred to as Hodge inner product. If $K \subset \mathbb{R}$, we require instead the existence of a non-degenerate, locally constant, antisymmetric form $Q(\cdot, \cdot)$ on $\mathbb{W}$, which is zero on $\mathcal{W}^{1,0} \otimes \mathcal{W}^{1,0}$ and on $\mathcal{W}^{0,1} \otimes \mathcal{W}^{0,1}$ and such that $i^{p-q} Q(v, \bar{v})>0$ for every non-zero $v \in \mathcal{W}^{p, q}$. The indefinite hermitian form on $\mathbb{W}_{\mathbb{C}}=\mathbb{W} \otimes_{K} \mathbb{C}$ is then given by

$$
\psi(v, w):=\frac{i}{2} Q(v, \bar{w})
$$

If $K \subset \mathbb{R}$, then we may write every element $w \in \mathbb{W}$ as $w=\operatorname{Re}(\omega)$ for some $\omega \in \mathcal{W}^{1,0}$ and define the Hodge norm as $\|w\|=\psi(\omega, \omega)^{1 / 2}$. We extend this norm to $\mathbb{W}_{\mathbb{C}}=\mathbb{W}_{\mathbb{R}}+i \mathbb{W}_{\mathbb{R}}$ as orthogonal direct sum and also to $\mathbb{C}$-sub-VHS. This Hodge norm will be used throughout when talking about Lyapunov exponents. In particular, for $v \in \mathbb{W}_{\mathbb{C}}$ we have

$$
\begin{aligned}
\|v\|^{2} & =\|\operatorname{Re}(v)\|^{2}+\|i \operatorname{Im}(v)\|^{2}=\psi\left((v+\bar{v})^{1,0},(v+\bar{v})^{1,0}\right)+\psi\left((v-\bar{v})^{1,0},(v-\bar{v})^{1,0}\right) \\
& =2\left(\psi\left(v^{1,0}, v^{1,0}\right)+\psi\left(\overline{v^{0,1}}, \overline{v^{0,1}}\right)\right)
\end{aligned}
$$

Suppose that we are given a polarized $\mathbb{R}$-VHS $\mathbb{W}$ that splits over $\mathbb{C}$ as $\mathbb{W}_{\mathbb{C}}=\mathbb{V} \oplus \overline{\mathbb{V}}$ into $\mathbb{C}$-VHS. Since $\psi(\bar{v}, \bar{v})=-\psi(v, v)$, the above computation shows that we may also flip the sign of $\psi$ on the negative definite part $\mathcal{V}^{0,1}$ in order to obtain a positive hermitian form that computes the Hodge norm of vectors in $\mathbb{V}$ :

$$
\|v\|^{2}=2\left(\psi\left(v^{1,0}, v^{1,0}\right)-\psi\left(v^{0,1}, v^{0,1}\right)\right) .
$$

If $f: \mathcal{X} \rightarrow B$ is a family of curves, then $\mathbb{W}=R^{1} f_{*} K$ is a $K$-VHS of weight one, polarized by the natural intersection pairing on cohomology.

\footnotetext{
${ }^{3} \mathrm{Be}$ aware that our definition of $\psi$ parallels the one used for the area of a flat surface (see e.g. [EKZ14]). In Hodge theory (e.g. [Sch73]), the factor $\frac{1}{2}$ is usually omitted.
} 
Let $W$ be a $\mathbb{C}$-vector space and let $\psi$ be a polarization. The period domain $\operatorname{Per}(W)$ is the classifying space of all $\mathbb{C}$-Hodge structures polarized by $\psi$ that can be put on $W$. Analogously, we define the period domain for $K$-Hodge structures on the $K$-vector space $W$, polarized by the $K$-bilinear form $Q$.

Note that for weight one, $\operatorname{Per}(W)$ is always a hermitian symmetric domain. In fact, weight one $\mathbb{C}$-Hodge structures of signature $(1, n)$ are parametrized by the complex ball $\mathbb{B}^{n}$, and weight one $\mathbb{R}$-Hodge structures are parametrized by the Siegel upper halfspace $\mathbb{H}_{\operatorname{dim} W}$.

Let $b \in B$ be a base point. A polarized VHS W defines a period $\operatorname{map} p: \hat{B} \rightarrow$ $\operatorname{Per}\left(\mathbb{W}_{b}\right)$ from the universal cover of $B$ to the period domain $\operatorname{Per}\left(\mathbb{W}_{b}\right)$ that records the position of the $(1,0)$-subspace in the fibers of $\mathbb{W}$. The period map is only well-defined on the universal cover, since the identification of the fibers of $\mathbb{W}$ by parallel transport depends on the chosen path, and in fact produces an action of the fundamental group $\pi_{1}(B, b)$ on $\mathbb{W}_{b}$, preserving the polarization. The group homomorphism $\rho: \pi_{1}(B, b) \rightarrow \operatorname{Aut}\left(\mathbb{W}_{b}, \psi_{b}\right)$ is called monodromy representation. There is an induced action of $\pi_{1}(B, b)$ on $\operatorname{Per}\left(\mathbb{W}_{b}\right)$ and the period map is equivariant with respect to the actions of $\pi_{1}(B, b)$ on the universal cover by deck transformations and on $\operatorname{Per}\left(\mathbb{W}_{b}\right)$. In particular, if $K$ denotes the Kernel of $\rho$, then $p$ drops to a map $\hat{B} / K \rightarrow \operatorname{Per}\left(\mathbb{W}_{b}\right)$. In the sequel, we will often omit the base point from the notation, if its choice does not matter.

Definition 2.3. A VHS is called uniformizing, if the period map is an isomorphism.

A uniformizing VHS is unique up to isomorphism. Indeed, if $\mathbb{W}_{i}, i=1,2$ are uniformizing VHS on $B$, we obtain a group isomorphism $\Gamma_{1} \rightarrow \Gamma_{2}$ of the monodromy groups and a biholomorphic map $\operatorname{Per}\left(\mathbb{W}_{1}\right) \rightarrow \operatorname{Per}\left(\mathbb{W}_{2}\right)$ equivariant with respect to this group isomorphism, which yields $\mathbb{W}_{1} \cong \mathbb{W}_{2}$ as VHS.

Suppose now that $\mathbb{V}$ is a $\mathbb{Q}$-VHS and that $\mathbb{V} \otimes_{\mathbb{Q}} \mathbb{C}$ contains a uniformizing subVHS $\mathbb{W}$ of signature $(1, n)$. The assumption on the signature implies that $\mathbb{W}$ is irreducible. Recall also that by Deligne's semisimplicity theorem [Del87, Prop. 1.13], $\mathbb{V}=\bigoplus_{i} \mathbb{W}_{i} \otimes E_{i}$, where $\mathbb{W}_{i}$ are irreducible, polarized $\mathbb{C}$-VHS and $E_{i}$ are $\mathbb{C}$-vector spaces.

Definition 2.4. We define the primitive part of a $\mathbb{Q}-V H S \mathbb{V}$ to be the direct sum of those $\mathbb{W}_{i} \otimes E_{i}$, where $\mathbb{W}_{i}$ is isomorphic as a local system to $\mathbb{W}^{\sigma}$ with $\sigma \in \operatorname{Aut}(\mathbb{C} / \mathbb{Q})$.

It is clear from the definition that the primitive part does not depend on the choice of a uniformizing sub-VHS, since any two such are isomorphic. Thus dropping the dependence on $\mathbb{W}$ will cause no confusion. The primitive part is fixed by any element of $\operatorname{Aut}(\mathbb{C} / \mathbb{Q})$, and thus a $\mathbb{Q}$-sub-VHS of $\mathbb{V}$.

For the application below we explicit the (unique up to isomorphism) uniformizing VHS $\mathbb{U}$ over $\mathbb{B}^{n}$ together with the polarization in terms of the form $\langle\cdot, \cdot\rangle_{1, n}$ used above. Over $\mathbb{B}^{n}$ we represent a constant $\mathbb{C}$-local system of rank $n+1$ by row vectors and define $\mathbb{U}_{z}^{1,0}$ as the line generated by $\underline{z}=(1, z)$ in $\{z\} \times \mathbb{C}^{n+1}$. Let $\pi_{z}$ denote the orthogonal projection on the 1,0 -part. Then for $\underline{v} \in\{z\} \times \mathbb{C}^{n+1}$

$$
\begin{aligned}
\frac{1}{2}\|\underline{v}\|_{z}^{2} & =\left\langle\pi_{z}(\underline{v}), \pi_{z}(\underline{v})\right\rangle_{1, n}-\left\langle\left(1-\pi_{z}\right)(\underline{v}),\left(1-\pi_{z}\right)(\underline{v})\right\rangle_{1, n} \\
& =-\langle\underline{v}, \underline{v}\rangle_{1, n}+2 \frac{\left.|\underline{\underline{v}}, \underline{z}\rangle_{1, n}\right|^{2}}{\langle\underline{z}, \underline{z}\rangle_{1, n}}
\end{aligned}
$$


2.2.1. The Higgs field. By a theorem of Borel (see e.g. SSch73, Lemma 4.5]), the local monodromy about a boundary divisor in $\Delta$ is always quasi-unipotent. In the sequel we suppose throughout that the monodromy of the local system $\mathbb{W}$ about $\Delta$ is already unipotent, or pass to a finite cover where this holds. Then the associated holomorphic vector bundle $\mathbb{W} \otimes_{\mathbb{C}} \mathcal{O}_{B}$ has a unique extension due to Deligne Del70, Prop. 5.2] to a holomorphic bundle $\mathcal{E}$ on $Y$. The extension $\mathcal{E}^{1,0}$ of $\mathcal{W}^{1,0}$ inside $\mathcal{E}$ is a holomorphic subbundle and we set $\mathcal{E}^{0,1}:=\mathcal{E} / \mathcal{E}^{1,0}$. Further let $\Omega_{Y}^{1}(\log \Delta)$ be the bundle of differential forms on $Y$ with logarithmic poles along $\Delta$; then $\omega_{Y}=\bigwedge^{\operatorname{dim} B} \Omega_{Y}^{1}(\log \Delta)$ is called the log-canonical bundle. The graded piece of the Gauss-Manin connection is an $\mathcal{O}_{Y}$-linear map

$$
\tau: \mathcal{E}^{1,0} \rightarrow \mathcal{E}^{0,1} \otimes \Omega_{Y}^{1}(\log \Delta),
$$

called the Higgs field. Its dual is the $\mathcal{O}_{Y \text {-linear map }}$

$$
\tau^{\vee}: \mathcal{E}^{1,0} \otimes \mathcal{T}_{Y}(-\log \Delta) \rightarrow \mathcal{E}^{0,1} \otimes \Omega_{Y}^{1}(\log \Delta) \otimes \mathcal{T}_{Y}(-\log \Delta) \rightarrow \mathcal{E}^{0,1}
$$

obtained by composing with the natural contraction. Another viewpoint on the same map is the map

$$
\widetilde{\tau}: \mathcal{T}_{Y}(-\log \Delta) \rightarrow \mathcal{H o m}\left(\mathcal{E}^{1,0}, \mathcal{E}^{0,1}\right)
$$

obtained from $\tau^{\vee}$ by tensoring with $\left(\mathcal{E}^{1,0}\right)^{*}$. If $p: \hat{B} \rightarrow \operatorname{Per}(\mathbb{W})$ denotes the period map of $\mathbb{W}$, then its differential descends to a sheaf map on $B$, which is equal to $\widetilde{\tau}$ restricted to $B$.

2.3. Lyapunov spectrum for polarized VHS. We quickly recall Oseledec's theorem together with some properties

Theorem 2.5 (see e.g. Rue79]). Let $g_{t}:(M, \mu) \rightarrow(M, \mu)$ be an ergodic flow on a space $M$ with finite measure $\mu$. Suppose that the action of $t \in \mathbb{R}$ lifts equivariantly to a linear flow $G_{t}$ on some measurable real bundle $V$ on $M$. Suppose there exists a (not equivariant) norm $\|\cdot\|$ on $V$ such that the functions

$$
x \mapsto \sup _{t \in[0,1]} \log ^{+}\left\|G_{t}\right\|_{x} \quad \text { and } \quad x \mapsto \sup _{t \in[0,1]} \log ^{+}\left\|G_{1-t}\right\|_{g_{t}(x)}
$$

are in $L^{1}(M, \mu)$. Then there exist real constants $\lambda_{1} \geq \cdots \geq \lambda_{k}$ and a decomposition

$$
V=\bigoplus_{i=1}^{k} V_{\lambda_{i}}
$$

by measurable vector subbundles such that, for almost all $m \in M$ and all $v \in$ $\left(V_{\lambda_{i}}\right)_{m} \backslash\{0\}$, one has

$$
\lambda_{i}=\lim _{t \rightarrow \pm \infty} \frac{1}{t} \log \left\|G_{t}(v)\right\| .
$$

We call the values $\lambda_{i}$, repeated with multiplicity $\operatorname{dim} V_{\lambda_{i}}$ the set of Lyapunov exponents or Lyapunov spectrum of $\left(M, \mu, V, g_{t}\right)$.

Suppose that $V$ carries a symplectic structure preserved by the flow. Then the Lyapunov exponents are symmetric with respect to zero, i.e. $\lambda_{k+1-i}=-\lambda_{i}$. The positive Lyapunov spectrum is by definition the first half of the symmetric Lyapunov spectrum.

The $V_{\lambda_{i}}$ do not change if $\|\cdot\|$ is replaced by another norm of 'comparable' size. More precisely, 
Lemma 2.6. In the above theorem, suppose $M$ is a locally compact topological space and $\mu$ a regular Borel measure. Let $\|\cdot\|_{1}$ and $\|\cdot\|_{2}$ be norms on $V$ (varying measurably) such that for both (14) is satisfied. Then the filtrations and the two sets of Lyapunov exponents coincide.

Proof. The function $m \mapsto \phi(m)=\sup _{\|v\|_{2, m}=1}\|v\|_{1, m}$ is measurable. Thus, by Lusin's theorem, there is a compact subset $K$ of positive measure and a $C=$ $C(K)>0$ such $\phi(m) \leq C$ for all $m \in K$. By Poincaré recurrence, we find for almost all $m$ an increasing sequence of times $t_{1}, t_{2}, \ldots$ with $\lim t_{j} \rightarrow \infty$ such that $g_{t_{j}}(m) \in K$ for all $j$. Now for almost all $m$ and $v \in V_{m} \backslash\{0\}$,

$$
\begin{aligned}
\lim _{t \rightarrow \infty} \frac{1}{t} \log \left\|G_{t}(v)\right\|_{1} & =\lim _{j \rightarrow \infty} \frac{1}{t_{j}} \log \left\|G_{t_{j}}(v)\right\|_{1} \leq \lim _{j \rightarrow \infty} \frac{1}{t_{j}} \log \left(C \cdot\left\|G_{t_{j}}(v)\right\|_{2}\right) \\
& =\lim _{j \rightarrow \infty} \frac{1}{t_{j}} \log \left\|G_{t_{j}}(v)\right\|_{2}=\lim _{t \rightarrow \infty} \frac{1}{t} \log \left\|G_{t}(v)\right\|_{2}
\end{aligned}
$$

By symmetry, we also obtain the other inequality, hence

$$
\lim _{t \rightarrow \infty} \frac{1}{t} \log \left\|G_{t}(v)\right\|_{1}=\lim _{t \rightarrow \infty} \frac{1}{t} \log \left\|G_{t}(v)\right\|_{2},
$$

and this implies the claim.

In all the applications below, the manifold $M$ will be the unit tangent bundle $T^{1} B$ of a ball quotient $B, g_{t}$ will be the geodesic flow, which is well-known to be ergodic, and $\mu$ will be the push-forward of Haar measure on $\mathrm{PU}(1, n) . V$ will be the pullback along $T^{1} B \rightarrow B$ of the vector bundle associated with a polarized $\mathbb{R}$-VHS $\mathbb{V}$, and will be endowed with the Hodge norm. The flat connection then provides a lift $G_{t}$ of the geodesic flow $g_{t}$ on the base $T^{1} B$. We first check the integrability condition.

Lemma 2.7. In this setting, the integrability condition (14) holds. More precisely, we have

$$
\left|\frac{\mathrm{d}}{\mathrm{d} t} \log \left\|G_{t}(v)\right\|\right| \leq \frac{1}{\sqrt{2 \kappa}}
$$

Proof. We first argue that it suffices to show (16). It clearly suffices to bound $\log \left\|G_{t}(v)\right\|$ for every $v$ of norm 1 by a constant depending continuously on $t \in \mathbb{R}$. Assuming (16), we have

$$
\left|\log \left\|G_{t}(v)\right\|\right|=\left|\int_{0}^{t} \frac{\mathrm{d}}{\mathrm{d} s} \log \left\|G_{s}(v)\right\| \mathrm{d} s\right| \leq \int_{0}^{t}\left|\frac{\mathrm{d}}{\mathrm{d} s} \log \left\|G_{s}(v)\right\|\right| \mathrm{d} s \leq t \frac{1}{\sqrt{2 \kappa}} .
$$

Let $\vartheta \in T^{1} B$, and let $\gamma:[0, t] \rightarrow B$ be the geodesic with $\gamma^{\prime}(0)=\vartheta$. For $v \in V_{\vartheta}$, the lift $G_{t}(v)$ of the flow is given by a flat section $w$ of $V$ along $\gamma$ with $w_{\vartheta}=v$. We will use the following formula, which is proved in [FMZ14a, Lemma 2.3] for the case of the Teichmüller geodesic flow; the proof carries over to our situation.

$$
\left.\frac{\mathrm{d}}{\mathrm{d} t} \psi\left(w_{\gamma(t)}^{1,0}, w_{\gamma(t)}^{1,0}\right)\right|_{t=t_{0}}=\left.2 \cdot \operatorname{Re}\left(\psi\left(\widetilde{\tau}_{\gamma^{\prime}(t)}\left(w_{\gamma(t)}^{1,0}\right), w_{\gamma(t)}^{0,1}\right)\right)\right|_{t=t_{0}} .
$$

Using $\left\|w_{\gamma(t)}\right\|^{2}=4 \psi\left(w_{\gamma(t)}^{1,0}, w_{\gamma(t)}^{1,0}\right)$, we have

$$
\begin{aligned}
\left.\frac{\mathrm{d}}{\mathrm{d} t} \log \left\|G_{t}(v)\right\|\right|_{t=t_{0}} & =\left.2\left\|G_{t_{0}}(v)\right\|^{-2} \cdot \frac{\mathrm{d}}{\mathrm{d} t} \psi\left(w_{\gamma(t)}^{1,0}, w_{\gamma(t)}^{1,0}\right)\right|_{t=t_{0}} \\
& =4\left\|G_{t_{0}}(v)\right\|^{-2} \cdot \operatorname{Re}\left(\psi\left(\widetilde{\tau}_{\gamma^{\prime}\left(t_{0}\right)}\left(w_{\gamma\left(t_{0}\right)}^{1,0}\right), w_{\gamma\left(t_{0}\right)}^{0,1}\right)\right),
\end{aligned}
$$


hence by applying the Cauchy-Schwarz inequality

$$
|\psi(x, y)| \leq \frac{1}{\sqrt{2}}\|x\| \cdot \frac{1}{\sqrt{2}}\|y\|
$$

on the $(0,1)$ subspace and using the submultiplicativity of operator norm $\|\cdot\|_{\text {op }}$ on $\operatorname{Hom}\left(V^{1,0}, V^{0,1}\right)$

$$
\begin{aligned}
\left|\frac{\mathrm{d}}{\mathrm{d} t} \log \left\|G_{t}(v)\right\|\right|_{t=t_{0}} \mid & \leq 2\left\|G_{t_{0}}(v)\right\|^{-2} \cdot\left\|\widetilde{\tau}_{\gamma^{\prime}\left(t_{0}\right)}\left(w_{\gamma\left(t_{0}\right)}^{1,0}\right)\right\| \cdot\left\|w_{\gamma\left(t_{0}\right)}^{0,1}\right\| \\
& \leq 2\left\|G_{t_{0}}(v)\right\|^{-2} \cdot\left\|\widetilde{\tau}_{\gamma^{\prime}\left(t_{0}\right)}\right\|_{\mathrm{op}} \cdot\left\|w_{\gamma\left(t_{0}\right)}^{1,0}\right\| \cdot\left\|w_{\gamma\left(t_{0}\right)}^{0,1}\right\| \\
& \leq\left\|\widetilde{\tau}_{\gamma^{\prime}\left(t_{0}\right)}\right\|_{\text {op }} .
\end{aligned}
$$

The map $\widetilde{\tau}$ can be identified with the derivative of the period map $p: \mathbb{B}^{n} \rightarrow \mathbb{H}_{\mathrm{rkV}}$ associated with $\mathbb{V}$. We may use the realization of $\mathbb{H}_{\mathrm{rk} \mathbb{V}}$ as symmetric matrices, so a tangent vector like $\widetilde{\tau}_{\gamma^{\prime}\left(t_{0}\right)}$ is also given by a symmetric matrix. Acting by an appropriate block diagonal element in the symplectic group, we may suppose that $\widetilde{\tau}_{\gamma^{\prime}\left(t_{0}\right)}$ is given by a diagonal matrix. To bound its operator norm it suffices to bound the operator norm of the individual diagonal entries. Let $r_{i}: \mathbb{H}_{\mathrm{rkV}} \rightarrow \mathbb{H}$ be the projection on the $i$-th diagonal element and $p_{i}=r_{i} \circ p: \mathbb{B}^{n} \rightarrow \mathbb{H}$. In this notation, we want to bound the operator norm of $\mathrm{d} r_{i}\left(\widetilde{\tau}_{\gamma^{\prime}}\left(t_{0}\right)\right)=\left(\mathrm{d} p_{i}\right)\left(\gamma^{\prime}\left(t_{0}\right)\right)$.

For this purpose we want to apply a generalization of the Schwarz-Pick Lemma (e.g. Roy80, Theorem 2]) to the composition $p_{i}$. The ball $\mathbb{B}^{n}$ is a hermitian manifold of constant negative holomorphic sectional curvature $k_{0}$, in fact with our normalization we have $k_{0}=\frac{-2}{\kappa}$. Suppose we take the hermitian metric of constant holomorphic sectional curvature $K_{0}$ on $\mathbb{H}$ and let $\|\cdot\|_{\mathbb{H}}$ be the associated norm. In this situation, Royden's theorem states that $\left\|\mathrm{d} p_{i}\right\|_{\mathbb{H}} \leq \sqrt{k_{0} / K_{0}}$. Now it suffices to check that for $K_{0}=-4$ the operator norm on the tangent bundle and the norm $\|\cdot\|_{\mathbb{H}}$ coincide.

To do so, we use the unit disk model $\mathbb{H} \cong \mathbb{B}^{1}$ of hyperbolic space. The norm $\|\cdot\|_{\mathbb{H}}$ is given by the hermitian form

$$
h=\frac{2 \kappa}{\left(1-|z|^{2}\right)^{2}} d z \otimes d \bar{z}, \quad \text { where } K_{0}=\frac{-2}{\kappa}
$$

and the operator norm on $T \mathbb{B}^{1}$ is induced by the isomorphism $T \mathbb{B}^{1} \rightarrow \operatorname{Hom}\left(E^{1,0}, E^{0,1}\right)$ coming from the Higgs field $\widetilde{\tau}=\widetilde{\tau}_{\mathbb{B}^{1}}$ of the uniformizing VHS on $\mathbb{B}^{1}$. Here, $E=\mathbb{B}^{1} \times \mathbb{C}^{2}$ is the trivial bundle with trivial connection

$$
\nabla\left(f_{1} e_{1}+f_{2} e_{2}\right)=d f_{1} \otimes e_{1}+d f_{2} \otimes e_{2}
$$

and indefinite hermitian form $\psi(v, w)=v_{1} \bar{w}_{1}-v_{2} \bar{w}_{2}$ with respect to the standard basis $\left\{e_{1}, e_{2}\right\} . E^{1,0}$ is the holomorphic subbundle with fiber $E_{z}^{1,0}=\mathbb{C}\left(e_{1}+z e_{2}\right)$ and $E^{0,1}$ is its orthogonal complement with fiber $E_{z}^{0,1}=\mathbb{C}\left(\bar{z} e_{1}+e_{2}\right)$. The norm on $E^{1,0}$ and $E^{0,1}$ is the one induced by restricting $\psi$ and flipping the sign on $E^{0,1}$, i.e.

$$
\left\|\lambda\left(e_{1}+z e_{2}\right)\right\|^{2}=|\lambda|^{2}\left(1-|z|^{2}\right)=\left\|\lambda\left(\bar{z} e_{1}+e_{2}\right)\right\|^{2} .
$$

For the identification $T \mathbb{B}^{1} \rightarrow \operatorname{Hom}\left(E^{1,0}, E^{0,1}\right)$, we first consider the map

$$
\partial_{z} \mapsto\left(\lambda\left(e_{1}+z e_{2}\right) \mapsto \nabla_{\partial_{z}}\left(\lambda\left(e_{1}+z e_{2}\right)\right)=\lambda_{z} e_{1}+\left(\lambda+z \lambda_{z}\right) e_{2}\right),
$$

where $\lambda_{z}=\frac{\partial \lambda}{\partial z}$. The orthogonal projection to $E^{0,1}$ is given by

$$
\begin{gathered}
f_{1} e_{1}+f_{2} e_{2} \mapsto \psi\left(f_{1} e_{1}+f_{2} e_{2}, \bar{z} e_{1}+e_{2}\right) \cdot \psi\left(\bar{z} e_{1}+e_{2}, \bar{z} e_{1}+e_{2}\right)^{-1} \cdot\left(\bar{z} e_{1}+e_{2}\right) \\
=\frac{1}{|z|^{2}-1}\left(f_{1} z-f_{2}\right)\left(\bar{z} e_{1}+e_{2}\right),
\end{gathered}
$$


and the Higgs field is the composition of these two maps. Thus,

$$
\begin{aligned}
\widetilde{\tau}\left(\partial_{z}\right)\left(\lambda\left(e_{1}+z e_{2}\right)\right) & =\frac{1}{|z|^{2}-1}\left(\lambda_{z} z-\left(\lambda+z \lambda_{z}\right)\right)\left(\bar{z} e_{1}+e_{2}\right) \\
& =\frac{\lambda}{1-|z|^{2}}\left(\bar{z} e_{1}+e_{2}\right)
\end{aligned}
$$

Hence, the operator norm of $\widetilde{\tau}\left(\partial_{z}\right)$ is given by

$$
\begin{aligned}
\left\|\widetilde{\tau}\left(\partial_{z}\right)\right\| & =\frac{\left\|\sigma\left(\partial_{z}\right)\left(\lambda\left(e_{1}+z e_{2}\right)\right)\right\|}{\|\left(\lambda\left(e_{1}+z e_{2}\right) \|\right.}=\frac{|\lambda| \sqrt{\left(1-|z|^{2}\right)}}{\left(1-|z|^{2}\right)} \cdot|\lambda|^{-1}\left(\sqrt{1-|z|^{2}}\right)^{-1} \\
& =\frac{1}{1-|z|^{2}}
\end{aligned}
$$

Therefore, $\widetilde{\tau}$ is an isometry iff $\kappa=\frac{1}{2}$, which proves the claim.

The hypothesis of Oseledec's theorem holds verbatim for $\mathbb{V}_{\mathbb{C}}$ and for the summands of a decomposition of the VHS into $\mathbb{V}_{\mathbb{C}}=\oplus \mathbb{W}_{i}$, replacing Siegel upper-half space by the respective period domain for the polarized VHS. In particular, it applies to the primitive part $\mathbb{P}_{\mathbb{C}} \subset \mathbb{V}_{\mathbb{C}}$. We call the corresponding part of the Lyapunov spectrum the primitive Lyapunov spectrum.

\section{Lyapunov exponents of BALL QUotients: Generalities}

From now on $B$ will be a ball quotient, i.e. the quotient of $\mathbb{B}^{n}$ by some cofinite discrete subgroup $\Gamma \subset \mathrm{PU}(1, n)$. In this section we prove the general results announced as Theorem 1.1

3.1. Normalization. Recall the construction and the metric of the uniformizing local system $\mathbb{U}$ from Section 2.2. The following proposition comprises the statement of Theorem 1.1 i).

Proposition 3.1. The Lyapunov exponents of a uniformizing polarized VHS $\mathbb{U}$ are

$$
\lambda_{1}, \lambda_{1}, \underbrace{0, \ldots, 0}_{2 n-2},-\lambda_{1},-\lambda_{1}
$$

where $\lambda_{1}=\frac{1}{\sqrt{2 \kappa}}$, and the Lyapunov exponents of any polarized VHS $\mathbb{V}$ on $B$ are all bounded by $\lambda_{1}$.

Proof. We can lift the whole situation to the universal cover and look at the geodesic flow on $T^{1} \mathbb{B}^{n}$ acting on $\mathbb{B}^{n} \times \mathbb{C}^{n+1}$ endowed with the metric from Section 2.2. Moreover, it suffices to compute the Lyapunov exponents for the point $\left(0, \frac{1}{\sqrt{2 \kappa}} e_{1}\right) \in$ $T^{1} \mathbb{B}^{n}$ since $\mathrm{PU}(1, n)$ acts transitively on $T^{1} \mathbb{B}^{n}$ and the measure $\mu_{T^{1} \mathbb{B}^{n}}$ is invariant under this action. The unit speed geodesic flow starting at $\left(0, \frac{1}{\sqrt{2 \kappa}} e_{1}\right)$ is given by

$$
g_{t} \cdot\left(0, \frac{1}{\sqrt{2 \kappa}} e_{1}\right)=\left(\tanh \left(\frac{t}{\sqrt{2 \kappa}}\right) e_{1},\left(1-\tanh ^{2}\left(\frac{t}{\sqrt{2 \kappa}}\right)\right) \frac{1}{\sqrt{2 \kappa}} e_{1}\right)
$$

Since the local system is trivial, the geodesic flow acts trivially on the fibers of $T^{1} \mathbb{B}^{n} \times \mathbb{C}^{n+1}$. However, the norm in this bundle varies. Now let $\underline{x}=\left(x_{1}, \ldots, x_{n+1}\right)$ in the fiber over $\left(0, \frac{1}{\sqrt{2 \kappa}} e_{1}\right)$. Set $\underline{z}_{t}=\left(1, z_{t}\right)$, where $z_{t}$ is the projection of $g_{t}$. $\left(0, \frac{1}{\sqrt{2 \kappa}} e_{1}\right)$ to $\mathbb{B}^{n}$. By (13),

$$
\begin{aligned}
\left\|G_{t} \cdot \underline{x}\right\|^{2}=\|\underline{x}\|_{z_{t}}^{2} & =-2\langle\underline{x}, \underline{x}\rangle_{1, n}+4\left|\left\langle\underline{x}, \underline{z}_{t}\right\rangle_{1, n}\right|^{2} \cdot\left(\left\langle\underline{z}_{t}, \underline{z}_{t}\right\rangle_{1, n}\right)^{-1} \\
& =-2\langle\underline{x}, \underline{x}\rangle_{1, n}+4\left|x_{1} \cosh \left(\frac{t}{\sqrt{2 \kappa}}\right)-x_{2} \sinh \left(\frac{t}{\sqrt{2 \kappa}}\right)\right|^{2}
\end{aligned}
$$


since

$$
\left\langle\underline{z}_{t}, \underline{z}_{t}\right\rangle_{1, n}=1-\tanh ^{2}\left(\frac{t}{\sqrt{2 \kappa}}\right)=\cosh ^{-2}\left(\frac{t}{\sqrt{2 \kappa}}\right)
$$

and

$$
\left\langle\underline{x}, \underline{z}_{t}\right\rangle_{1, n}=x_{1}-x_{2} \tanh \left(\frac{t}{\sqrt{2 \kappa}}\right) .
$$

As $\cosh (\alpha) \pm \sinh (\alpha)=e^{ \pm \alpha}$, we see that

$$
\begin{gathered}
V^{\lambda_{1}}=[(1,-1,0, \ldots, 0),(i,-i, 0, \ldots, 0)]_{\mathbb{R}} \\
V^{0}=\left\{\underline{x} \in \mathbb{C}^{n, 1} \mid x_{1}=x_{2}=0\right\} \\
V^{-\lambda_{1}}=[(1,1,0, \ldots, 0),(i, i, 0, \ldots, 0)]_{\mathbb{R}}
\end{gathered}
$$

is an orthogonal decomposition of the fiber of $\mathbb{B}^{n} \times \mathbb{C}^{n+1}$ over $\left(0, \frac{1}{\sqrt{2 \kappa}} e_{1}\right)$ which realizes the Lyapunov exponents.

The second claim follows from Lemma 2.7, and its proof. First, we can assume $\mathbb{V}$ to be an $\mathbb{R}$-VHS, since we otherwise consider the real form of $\mathbb{V} \oplus \overline{\mathbb{V}}$, which has the same Lyapunov exponents as $\mathbb{V}$ (but possibly with different multiplicity). With curvature $k_{0}=\frac{-2}{\kappa}$ on the ball, we have the bound

$$
\frac{1}{t} \log \left\|G_{t}(v)\right\|=\frac{1}{t} \int_{0}^{t} \frac{\mathrm{d}}{\mathrm{d} s} \log \left\|G_{s}(v)\right\| \mathrm{d} s \leq \frac{1}{\sqrt{2 \kappa}} .
$$

3.2. Duplication. When working with $\mathbb{C}$-variations, there are two duplication phenomena of the Lyapunov spectrum. The first one is obvious from the fact that in a normed $\mathbb{C}$-vector space $V$, a vector $v \neq 0$ and $i v$ are $\mathbb{R}$-linearly independent and have the same norm. The second one occurs when an $\mathbb{R}$-VHS becomes reducible over $\mathbb{C}$.

Proposition 3.2. Let $\mathbb{W}_{\mathbb{R}}$ be an irreducible direct summand as in Theorem 1.1 that splits after tensoring with $\mathbb{C}$. Then each Lyapunov exponent of $\mathbb{W}_{\mathbb{R}}$ occurs with even multiplicity.

Proof. We have $\mathbb{W}_{\mathbb{R}} \otimes \mathbb{C}=\mathbb{V} \oplus \overline{\mathbb{V}}$ with a $\mathbb{C}$-variation $\mathbb{V}$. Therefore, the Lyapunov spectrum of $\mathbb{W} \otimes \mathbb{C}$ is the union of the two spectra of $\mathbb{V}$ and $\mathbb{V}$, and is the spectrum of $\mathbb{W}_{\mathbb{R}}$ with each exponent occurring with twice the multiplicity. Since $\|\bar{v}\|=\|v\|$ for each section $v$ of $\mathbb{V}$, and $G_{t} \cdot \bar{v}=\overline{G_{t} \cdot v}$, it follows that $\mathbb{V}$ and $\overline{\mathbb{V}}$ have the same Lyapunov spectrum, and each individual exponent occurs with even multiplicity because of the first duplication phenomenon.

3.3. Zero exponents. This result is probably well-known, it was observed for the Teichmüller geodesic flow in [FMZ14b] and we sketch their argument.

Proof of Theorem 1.1 iii). First, with respect to the indefinite hermitian form $\psi$, the Oseledec subspaces $V_{\lambda_{i}}$ are isotropic unless $\lambda_{i}=0$ and pairwise orthogonal unless $\lambda_{i}=-\lambda_{j}$. Indeed,

$$
\left|Q\left(v_{i}, v_{j}\right)\right| \leq c(K)\left\|v_{i}\right\|\left\|v_{j}\right\|
$$

on any compact set $K$ of positive measure with a uniform constant depending only on $K$, and

$$
\left\|G_{t} \cdot v_{i}\right\|\left\|G_{t} \cdot v_{j}\right\| \sim \exp \left(\left(\lambda_{i}+\lambda_{j}\right) t\right)
$$


which tends to zero for $t \rightarrow \infty$ or $t \rightarrow-\infty$. Since $g_{t}$ returns to $K$ for a sequence of times $t_{k} \rightarrow \infty$ or $t_{k} \rightarrow-\infty$

$$
Q\left(v_{i}, v_{j}\right)=Q\left(G_{t_{k}} \cdot v_{i}, G_{t_{k}} \cdot v_{j}\right) \rightarrow 0 .
$$

The key observation is now that an isotropic subspace in a vector space with an indefinite hermitian form of signature $(p, q)$ has at most dimension $\min (p, q)$. Since both $\bigoplus_{\lambda_{i}>0} V_{\lambda_{i}}$ and $\bigoplus_{\lambda_{i}<0} V_{\lambda_{i}}$ are isotropic, hence of dimension at most $\min (p, q)$, the complement $V_{0}$ has to be of dimension at least $|p-q|$.

Remark. The key observation of the proof also implies that the that the Lyapunov spectrum is symmetric with respect to 0 [FMZ14b, Lemma A.3]. Non-degeneracy of the Hodge inner product implies that the Lyapunov spectrum containing $\lambda_{i}$ also contains $-\lambda_{i}$. For $\lambda_{i} \neq 0, V_{\lambda_{i}} \oplus V_{-\lambda_{i}}$ is an orthogonal factor of the Oseledec decomposition, both of whose summands are isotropic, and hence have the same dimension. Therefore $\lambda_{i}=-\lambda_{p+q+1-i}$.

3.4. Partial sums are intersection numbers. We restate Part iv) of Theorem 1.1 for holomorphic sectional curvature $\frac{-2}{\kappa}$. Consider a polarized $\mathbb{R}-\mathrm{VHS} \mathbb{W}_{\mathbb{R}}$ on a ball quotient $B=\mathbb{B}^{n} / \Gamma$ as in Theorem $\left.1.1 \mathrm{iv}\right)$.

Theorem 3.3. The positive Lyapunov exponents $\lambda_{1}, \ldots, \lambda_{k}$ of $\mathbb{W}_{\mathbb{R}}$ satisfy

$$
\lambda_{1}+\cdots+\lambda_{k}=\frac{1}{\sqrt{2 \kappa}} \cdot \frac{(n+1) \mathrm{c}_{1}\left(\mathcal{E}^{1,0}\right) \cdot \mathrm{c}_{1}\left(\omega_{\bar{B}}\right)^{n-1}}{\mathrm{c}_{1}\left(\omega_{\bar{B}}\right)^{n}} .
$$

Before we engage in the proof, a few remarks are in order. Almost all ingredients are just parallel to [KZ97, see also [For02, explained in more detail in BM10] for the curve case and EKZ14] in general. There are a few modifications however.

First, we remark that the formula (17) is valid not only for (flat) surfaces, but for general weight one VHS. Second, the Laplacian on the $n$-ball replaces the Laplacian on the one-dimensional ball without major difficulties. Finally, at a crucial step we use that ball quotients are Kähler-Einstein manifolds to trade the first Chern class of the cotangent bundle for the class of the Kähler metric.

To start with the details, let $\Omega=\frac{1}{k !} \wedge^{k} Q \in\left(\bigwedge^{2 k} \mathbb{W}_{\mathbb{C}}\right)^{*}$. Consider a local section $L$ of $\bigwedge^{k} \mathbb{W}$ over an open set $U$ that is given by a decomposable vector $L=v_{1} \wedge \cdots \wedge v_{k}$. Such a vector is called Lagrangian, if the symplectic form $Q\left(v_{i}, v_{j}\right)=0$ for all pairs $(i, j)$. For a Lagrangian decomposable vector we claim that the Hodge norm can be calculated (see [EKZ14] and in more detail in [GH14]) by the formula

$$
\|L\|^{2}=\frac{\left|\Omega\left(\omega_{1} \wedge \cdots \wedge \omega_{k} \wedge v_{1} \wedge \cdots \wedge v_{k}\right)\right| \cdot\left|\Omega\left(v_{1} \wedge \cdots \wedge v_{k} \wedge \bar{\omega}_{1} \wedge \cdots \wedge \bar{\omega}_{k}\right)\right|}{\left|\Omega\left(\omega_{1} \wedge \cdots \wedge \omega_{k} \wedge \bar{\omega}_{1} \wedge \cdots \wedge \bar{\omega}_{k}\right)\right|}
$$

where $\omega_{1}, \ldots, \omega_{k}$ is a local basis of $\mathcal{W}^{1,0}$. Note that the right hand side does not depend on the choice of this basis.

Lemma 3.4. Given a decomposable Lagrangian section $L$ of $\bigwedge^{k} \mathbb{W}$ as above over some open subset $U$ of $B$, we have on $U$ the equality of functions

$$
\Phi:=\Delta_{\text {hyp }}(\log \|L\|)=-\frac{1}{2} \Delta_{\text {hyp }} \log \operatorname{det}\left(\psi\left(\omega_{i}, \omega_{j}\right)\right)_{i, j} .
$$

Proof. The proof is the same as in EKZ14 and the other sources above. The only thing we need to use is that for higher-dimensional balls, too, the hyperbolic Laplacian is a sum of $\frac{\partial^{2}}{\partial_{i} \bar{\partial}_{j}}$-derivatives and of $\frac{\partial^{2}}{\bar{\partial}_{i} \partial_{j}}$-derivatives by (8). Alternatively, 
one can use the proportionality (9) of $\Delta_{\text {hyp }}(\log \|L\|) \operatorname{dvol}_{\mathbb{B}^{n}}$ and $\partial \bar{\partial} \log \|L\| \wedge \omega_{\text {hyp }}^{n-1}$ and the fact that $\partial \bar{\partial} f=0$ for any holomorphic or antiholomorphic function $f$.

Since both sides of the equation do not depend of the choice of the basis we may consider $\Phi_{k}$ as a function on the whole of $B$.

Proof of Theorem 3.3. We first set up some notations. Let $T^{1} B$ be the unit tangent bundle, and let $\operatorname{Gr}_{k}(B)$ be the Lagrangian Grassmannian bundle over $B$, whose fiber over $x \in B$ consists of the $k$-dimensional Lagrangian $\mathbb{R}$-vector subspaces of $\mathbb{W}$. Set

$$
\operatorname{Gr}_{k}\left(T^{1} B\right)=T^{1} B \times{ }_{B} \operatorname{Gr}_{k}(B) .
$$

The Lagrangian Grassmannian is a homogeneous space, thus it carries a natural measure $\gamma$. The bundle measure $\mu_{\operatorname{Gr}_{k}\left(T^{1} B\right)}$ is then the product measure of $\gamma$ and the measure $\mu_{T^{1} B}$ on the base. We assume the measure $\gamma$, and the measure on the fiber of $T^{1} B$ to be normalized to have area 1. Denote again $G_{t}: \operatorname{Gr}_{k}\left(T^{1} B\right) \rightarrow \operatorname{Gr}_{k}\left(T^{1} B\right)$ the lift of the geodesic flow $g_{t}: T^{1} B \rightarrow T^{1} B$, and let $u: \mathbb{B}^{n} \rightarrow B$ be the universal covering map. If $\vartheta \in T^{1} B$, then we denote the coordinate on the base by $x(\vartheta) \in B$, and a lift to the universal cover by $\widetilde{x}(\vartheta)$.

The core of the proof is the following chain of equalities. After averaging over the whole space, we introduce another average over the unit tangent space at each point. Then we interchange the integral and the limit; this is possible, since the logarithmic derivative of the Hodge norm is bounded above by Lemma 2.7. Next, in order to apply Lemma 2.2. we pass to the universal cover. Then we use Lemma 3.4 to get rid of the dependence on $L$. Then we interchange once more limit and integral. Next, we go back to $B$ and decompose the fiber bundle $T^{\|\cdot\| \leq t} B$ along the fibers of

$$
\exp : T^{\|\cdot\| \leq t} B \rightarrow B \text {. }
$$

The preimage of $z \in B$ under this map has volume equal to the one of $\mathbb{B}_{t}^{n}(\widetilde{z})$, where $\widetilde{z}$ is a lift of $z$ to $\mathbb{B}^{n}$, since

$$
\begin{aligned}
\exp ^{-1}(z) & =\left\{\vartheta \in T^{\|\cdot\| \leq t} B \mid \exp (\vartheta)=z\right\} \\
& =\left\{\widetilde{\vartheta} \in T^{\|\cdot\| \leq t} \mathbb{B}^{n} \mid \exp (\widetilde{\vartheta})=\widetilde{z}\right\} \\
& =\bigcup_{0 \leq \tau \leq t} \bigcup_{d_{\mathrm{hyp}}(\widetilde{z}, y)=\tau}\{y\}
\end{aligned}
$$

Finally, the factor

$$
c_{1}(t)=\frac{\sqrt{2 \kappa}}{2 n} \tanh \left(\frac{t}{\sqrt{2 \kappa}}\right) \cdot \frac{1}{\operatorname{vol}\left(\mathbb{B}_{t}^{n}\right)}
$$

will drop out once we apply Lemma 2.2. In the last line we use that

$$
\frac{1}{T} \int_{0}^{T} \operatorname{vol}\left(\mathbb{B}_{t}^{n}\right) \cdot c_{1}(t) \mathrm{d} t=\frac{\kappa}{n} \cdot \frac{\log \left(\cosh \left(\frac{T}{\sqrt{2 \kappa}}\right)\right)}{T} \rightarrow \frac{\kappa}{n \cdot \sqrt{2 \kappa}}
$$

as $T \rightarrow \infty$.

$$
\begin{aligned}
\operatorname{vol}(B) \sum_{i=1}^{k} \lambda_{i} & =\int_{\operatorname{Gr}_{k}\left(T^{1} B\right)} \lim _{T \rightarrow \infty} \frac{1}{T} \int_{0}^{T} \frac{\mathrm{d}}{\mathrm{d} t} \log \left\|G_{t}(\vartheta, L)\right\| \mathrm{d} t \mathrm{~d} \mu_{\operatorname{Gr}_{k}\left(T^{1} B\right)}(\vartheta, L) \\
& =\int_{\operatorname{Gr}_{k}\left(T^{1} B\right)} \frac{1}{\sigma_{2 n-1}} \int_{T_{x(\vartheta)}^{1} B} \lim _{T \rightarrow \infty} \frac{1}{T} \int_{0}^{T} \frac{\mathrm{d}}{\mathrm{d} t} \log \left\|G_{t}(\theta, L)\right\|
\end{aligned}
$$




$$
\begin{aligned}
& \mathrm{d} t \mathrm{~d} \sigma(\theta) \mathrm{d} \mu_{\operatorname{Gr}_{k}\left(T^{1} B\right)}(\vartheta, L) \\
& =\int_{\operatorname{Gr}_{k}\left(T^{1} B\right)} \lim _{T \rightarrow \infty} \frac{1}{T} \int_{0}^{T} \frac{1}{\sigma_{2 n-1}} \frac{\mathrm{d}}{\mathrm{d} t} \int_{T_{x(\vartheta)}^{1} B} \log \left\|G_{t}(\theta, L)\right\| \\
& \mathrm{d} \sigma(\theta) \mathrm{d} t \mathrm{~d} \mu_{\operatorname{Gr}_{k}\left(T^{1} B\right)}((x, \vartheta), L) \\
& =\int_{\operatorname{Gr}_{k}\left(T^{1} B\right)} \lim _{T \rightarrow \infty} \frac{1}{T} \int_{0}^{T} \frac{1}{\sigma_{2 n-1}} \frac{\mathrm{d}}{\mathrm{d} t} \int_{T_{\widetilde{x}(\vartheta)}^{1} \mathbb{B}^{n}} \log \left\|G_{t}(u(\widetilde{\theta}), L)\right\| \\
& \mathrm{d} \sigma(\widetilde{\theta}) \mathrm{d} t \mathrm{~d} \mu_{\operatorname{Gr}_{k}\left(T^{1} B\right)}(\vartheta, L) \\
& =\int_{\operatorname{Gr}_{k}\left(T^{1} B\right)} \lim _{T \rightarrow \infty} \frac{1}{T} \int_{0}^{T} c_{1}(t) \int_{0}^{t} \int_{T_{\widetilde{x}(\vartheta)}^{1} \mathbb{B}^{n}} \Delta_{\text {hyp }} \log \left\|G_{\tau}(u(\widetilde{\theta}), L)\right\| \\
& \mathrm{d} \sigma(\widetilde{\theta}) \mathrm{d} \tau \mathrm{d} t \mathrm{~d} \mu_{\operatorname{Gr}_{k}\left(T^{1} B\right)}(\vartheta, L) \\
& =\int_{B} \lim _{T \rightarrow \infty} \frac{1}{T} \int_{0}^{T} c_{1}(t) \int_{0}^{t} \int_{T_{\widetilde{x}}^{1} \mathbb{B}^{n}}-\frac{1}{2} \Delta_{\text {hyp }} \log \operatorname{det}\left(\psi\left(\omega_{i}, \omega_{j}\right)\right)_{i, j}\left(u\left(\exp _{\widetilde{x}}(\tau \widetilde{\theta})\right)\right) \\
& \mathrm{d} \sigma(\widetilde{\theta}) \mathrm{d} \tau \mathrm{d} t \mathrm{~d} \mu_{B}(x) \\
& =\lim _{T \rightarrow \infty} \frac{1}{T} \int_{0}^{T} c_{1}(t) \int_{B} \int_{0}^{t} \int_{T_{\widetilde{x}}^{1} \mathbb{B}^{n}}-\frac{1}{2} \Delta_{\text {hyp }} \log \operatorname{det}\left(\psi\left(\omega_{i}, \omega_{j}\right)\right)_{i, j}\left(u\left(\exp _{\widetilde{x}}(\tau \widetilde{\theta})\right)\right) \\
& \mathrm{d} \sigma(\widetilde{\theta}) \mathrm{d} \tau \mathrm{d} \mu_{B}(x) \mathrm{d} t \\
& =\lim _{T \rightarrow \infty} \frac{1}{T} \int_{0}^{T} c_{1}(t) \int_{T\|\cdot\| \leq t} \Phi\left(\exp _{x(v)}(v)\right) \mathrm{d} \mu_{T\|\cdot\| \leq t}(v) \mathrm{d} t \\
& =\lim _{T \rightarrow \infty} \frac{1}{T} \int_{0}^{T} c_{1}(t) \int_{B} \int_{\exp ^{-1}(z)} \Phi(z) \mathrm{d} \mu_{\exp ^{-1}(z)} \mathrm{d} \mu_{B}(z) \mathrm{d} t \\
& =\int_{B} \Phi(z) \mathrm{d} \mu_{B}(z) \cdot \lim _{T \rightarrow \infty} \frac{1}{T} \int_{0}^{T} c_{1}(t) \int_{\exp ^{-1}(z)} \mathrm{d} \mu_{\exp ^{-1}(z)} \mathrm{d} t \\
& =\int_{B} \Phi(z) \mathrm{d} \mu_{B}(z) \cdot \lim _{T \rightarrow \infty} \frac{1}{T} \int_{0}^{T} c_{1}(t) \operatorname{vol}\left(\mathbb{B}_{t}^{n}\right) \mathrm{d} t \\
& =\int_{B} \frac{\kappa}{n \sqrt{2 \kappa}} \Phi(x) \mathrm{d} \mu_{B}(x) \text {. }
\end{aligned}
$$

The measure $\mathrm{d} \mu_{B}$ is given by integrating against the volume form $\mathrm{dvol}_{B}$, which is the image of $\operatorname{dvol}_{\mathbb{B}^{n}}$ via the universal cover $u: \mathbb{B}^{n} \rightarrow B$. Choose a fundamental domain $\mathcal{F} \subset \mathbb{B}^{n}$ for the action of $\Gamma$. Let $F: B \rightarrow \mathbb{R}$ be the function

$$
F=\log \operatorname{det}\left(\psi\left(\omega_{i}, \omega_{j}\right)\right)_{i, j} .
$$

Then Lemma 2.1 yields

$$
\begin{aligned}
\frac{\kappa}{n \sqrt{2 \kappa}} \int_{B} \Phi(x) \mathrm{d} \mu_{B}(x) & =\frac{\kappa}{n \sqrt{2 \kappa}} \int_{\mathcal{F}}-\frac{1}{2} \Delta_{\text {hyp }}(F \circ u) \mathrm{dvol}_{\mathbb{B} n} \\
& =-\frac{i \kappa}{n ! \sqrt{2 \kappa}} \int_{\mathcal{F}} \partial \bar{\partial}(F \circ u) \wedge \omega_{\text {hyp }}^{n-1} .
\end{aligned}
$$

A ball quotient $B$ is a Kähler-Einstein manifold, therefore the first Chern class and the Kähler class are proportional. Moreover, as was remarked by B. Hunt 
Hun00, Lemma 1.8], the Kähler-Einstein metric also computes the logarithmic Chern class in case $B$ is not compact. The proportionality constant is determined from the holomorphic section curvature. By [Huy05, p. 223],

$$
\mathrm{c}_{1}\left(\omega_{\bar{B}}\right)=\left[-1 /(2 \pi) \cdot \operatorname{Ric}\left(B, g_{\mathrm{hyp}}\right)\right]
$$

and by [KN96, p. 168]

$$
\operatorname{Ric}\left(B, g_{\text {hyp }}\right)=\frac{1}{2}(n+1) k_{0} \omega_{\text {hyp }},
$$

where $k_{0}=\frac{-2}{\kappa}$ is the holomorphic sectional curvature. Thus,

$$
\mathrm{c}_{1}\left(\omega_{\bar{B}}\right)=\frac{(n+1)}{2 \pi \kappa}\left[\omega_{\text {hyp }}\right] .
$$

As the metric on $\mathcal{W}^{1,0}$ is good in the sense of Mumford (by Schmid's $\mathrm{SL}_{2}(\mathbb{R})$ orbit theorem, e.g. CKS86. Theorem 5.21]), it computes the first Chern class of Mumford's extension of $\mathcal{W}^{1,0}$ to $\bar{B}$, which is the same as the Deligne extension, see e.g. [MVZ12, Lemma 3.4]. Consequently,

$$
\mathrm{c}_{1}\left(\mathcal{E}^{1,0}\right)=\frac{i}{2 \pi}\left[\Theta\left(\wedge^{k} \mathcal{W}^{1,0}\right)\right],
$$

where the curvature $\Theta\left(\wedge^{k} \mathcal{W}^{1,0}\right)$ is given by $-\partial \bar{\partial} F$. Altogether we obtain

$$
\begin{aligned}
\operatorname{vol}(B) \sum_{i=1}^{k} \lambda_{i} & =\frac{1}{\sqrt{2 \kappa}} \cdot \frac{i \kappa}{n !} \cdot \frac{2 \pi}{i} \cdot\left(\frac{2 \pi \kappa}{(n+1)}\right)^{n-1} \cdot \mathrm{c}_{1}\left(\mathcal{E}^{1,0}\right) \cdot \mathrm{c}_{1}\left(\omega_{\bar{B}}\right)^{n-1}[B] \\
& =\frac{1}{\sqrt{2 \kappa}} \cdot \frac{(2 \pi \kappa)^{n}}{n !(n+1)^{n-1}} \mathrm{c}_{1}\left(\mathcal{E}^{1,0}\right) \cdot \mathrm{c}_{1}\left(\omega_{\bar{B}}\right)^{n-1}[B]
\end{aligned}
$$

On the other hand,

$$
\left[\mathrm{dvol}_{B}\right]=\frac{1}{n !}\left[\omega_{\mathrm{hyp}}\right]^{n}=\frac{(2 \pi \kappa)^{n}}{n !(n+1)^{n}} \mathrm{c}_{1}\left(\omega_{\bar{B}}\right)^{n} .
$$

This finishes the proof.

\section{INVARIANTS OF MODULAR EMBEDDINGS AND COMMENSURABILITY INVARIANTS}

The trace field of a lattice $\Gamma \subset \mathrm{PU}(1, n)$ is defined to be $E=\mathbb{Q}(\operatorname{tr}(\operatorname{Ad}(\gamma)), \gamma \in$ $\Gamma)$, where Ad is the adjoint representation. The trace field is an invariant of the commensurability class of $\Gamma$ (e.g. [DM86, Proposition 12.2.1]).

The aim of this section is to show that, among lattices $\Gamma \subset \mathrm{PU}(1, n)$ (or rather $\mathrm{SU}(1, n)$ for that matter) that admit a modular embedding, the Lyapunov spectrum and the relative orbifold Euler characteristics are also commensurability invariants. In fact, given a modular embedding, we can define several invariants using intersection theory and we have shown in Theorem 1.1 resp. we will show in Corollary 8.2 later that the two invariants mentioned above, the Lyapunov spectrum and the relative orbifold Euler characteristics, indeed arise in that way. At the end of this section we give another proof of the commensurability invariance of the Lyapunov spectrum without referring to modular embeddings. 
Remark. From the point of view of Lyapunov exponents, arithmetic lattices in $\mathrm{PU}(1, n)$ are less interesting, as we will show in the next section.

The construction of non-arithmetic lattices in $\mathrm{PU}(1, n)$ is a long-standing challenge. Besides the cyclic coverings studied below there are several techniques to construct (non-arithmetic) lattices in $\mathrm{PU}(1, n)$, notably the original construction using complex reflection groups by Mostow ([Mos80]). By the work of Sauter ([Sau90]) and Deligne-Mostow (DM93) all the presently known non-arithmetic lattices in $\mathrm{PU}(1, n)$ turn out to be commensurable to lattices arising from cyclic coverings. See [Par09] for a recent survey on commensurability results and DPP11] for other constructions that by numerical evidence are very likely to give finitely many other non-arithmetic lattices.

4.1. Modular embeddings. In the following, we suppose that $\Gamma \subset \mathrm{SU}(1, n) \cap$ $\mathrm{GL}_{n+1}(F)$ for some number field $F$, which we take to be Galois over $\mathbb{Q}$. In particular, the Zariski closure of $\Gamma$, which by Borel density is all of $\mathrm{SU}(1, n)$, is defined over $F$, as is the adjoint representation. This entails $E \subseteq F$.

We say that $\Gamma$ admits a modular embedding, if for any $\sigma \in \operatorname{Gal}(F / \mathbb{Q})$

a) the Galois conjugate group $\Gamma^{\sigma}$ fixes a hermitian form of signature $(p(\sigma), q(\sigma))$, where we assume that $p(\sigma) \geq q(\sigma)$, and

b) there is a holomorphic map $\varphi^{\sigma}: \mathbb{B}^{n} \rightarrow \mathbb{B}_{p(\sigma), q(\sigma)}$ to the symmetric space of $\mathrm{PU}(p(\sigma), q(\sigma))$ that is equivariant with respect to the action of $\Gamma$ on the domain and $\Gamma^{\sigma}$ on the range of $\varphi^{\sigma}$.

We will show below that a lattice as above admits at most one modular embedding. Namely, the signature $(p(\sigma), q(\sigma))$ is uniquely determined by ( $\Gamma$ and) $\sigma$ by Lemma 4.1 below, and the map $\varphi^{\sigma}$ is unique by Theorem 4.3 .

In passing, we note that $p(\sigma)=0$ or $q(\sigma)=0$ implies that $\mathbb{B}_{p(\sigma), q(\sigma)}$ is a point, thus $\varphi^{\sigma}=$ const trivially does the job.

Our notion of modular embedding is essentially the same as in [CW93. Modular embeddings are also discussed in [McM13, Sec. 10]; however one should be aware that the target space of the period map used there is not equal to the period domain for signatures $(p, q)$ with $p+q=n+1,|p-q|<n-1$.

Lemma 4.1. Let $\Gamma$ and $\sigma$ be as above. Then $\Gamma^{\sigma}$ preserves a hermitian form for at most one signature $(p(\sigma), q(\sigma))$ with $p(\sigma) \geq q(\sigma)$.

Proof. Suppose the contrary holds. Then the Zariski closure of $\Gamma^{\sigma}$ is contained in the intersection of two special unitary groups $\mathrm{SU}\left(p_{1}, q_{1}\right)$ and $\mathrm{SU}\left(p_{2}, q_{2}\right)$ with $p_{i}+q_{i}=n+1, p_{i} \leq q_{i}$ but $p_{1} \neq q_{1}$. These two groups have both the (real) dimension $(n+1)^{2}-1$ and since they are not equal, their intersection has strictly smaller dimension. The dimension of the Zariski closure is invariant under Galois conjugation and the Zariski closure of $\Gamma$ over $\mathbb{R}$ is all of $\mathrm{SU}(1, n)$, hence of dimension $(n+1)^{2}-1$. This is a contradiction.

Now we prove the main result about modular embeddings. First, we show that we can pass to a finite index subgroup to realize the trace field as field of traces with respect to the standard embedding.

Lemma 4.2. There exists a finite index subgroup $\Gamma_{1} \subset \Gamma \subset \mathrm{SU}(1, n)$, such that the trace field $E=\mathbb{Q}(\operatorname{tr}(\operatorname{Ad}(\gamma)), \gamma \in \Gamma)$ for the adjoint representation of $\Gamma$ coincides with the trace field $\mathbb{Q}\left(\operatorname{tr}(\gamma), \gamma \in \Gamma_{1}\right)$ for the standard representation of $\Gamma_{1}$. 
Proof. By the argument given in [McR11, Appendix 2] it suffices to take $\Gamma_{1}$ as the kernel of the map from $\Gamma$ to its maximal abelian quotient of exponent $n+1$.

Theorem 4.3. Suppose $\Gamma \subset \mathrm{SU}(1, n)$ is a lattice with Galois conjugate $\Gamma^{\sigma} \subset$ $\mathrm{SU}(p(\sigma), q(\sigma))$ and we are given two maps $\varphi_{i}: \mathbb{B}^{n} \rightarrow \mathbb{B}_{p(\sigma), q(\sigma)}, i=1,2$ equivariant with respect to the action of $\Gamma$ and $\Gamma^{\sigma}$ on domain and range. Then $\varphi_{1}=\varphi_{2}$.

If $\tau$ fixes $E$ and $\varphi_{1}$ is equivariant with respect to $\Gamma$ and $\Gamma^{\sigma}$ and $\varphi_{2}$ is equivariant with respect to $\Gamma$ and $\Gamma^{\sigma \tau}$, then the signatures of the hermitian forms preserved by $\Gamma^{\sigma}$ and $\Gamma^{\sigma \tau}$ coincide and, moreover, there exists an automorphism $M$ of $\mathbb{B}_{p(\sigma), q(\sigma)}$ such that $M \circ \varphi_{1}=\varphi_{2}$.

Note that this statement is somewhat stronger than usual rigidity results for VHS (see e.g. [PS03, Corollary 12]) that require a priori that the two period maps coincide at one point.

Proof. The case $p(\sigma)=0$ or $q(\sigma)=0$ is trivial, since the range of $\varphi_{i}$ is just a point in this case.

Assume first that $\varphi_{i}$ is constant. Then $\Gamma^{\sigma}$ is entirely contained in the stabilizer of a point in $\mathrm{SU}(p(\sigma), q(\sigma))$. Thus, the Zariski-closure of $\Gamma^{\sigma}$ has strictly smaller dimension than $\mathrm{SU}(p(\sigma), q(\sigma))$. But on the other hand, this dimension is the same as the dimension of the Zariski-closure of $\Gamma$, which is $\mathrm{SU}(1, n)$, and we arrive at a contradiction. Thus, both $\varphi_{i}$ are not constant.

We consider the modular embeddings $\varphi_{i}$ as maps to the Harish-Chandra realization of $\mathbb{B}_{p(\sigma), q(\sigma)}$ as a bounded symmetric domain. Being composed of bounded holomorphic functions, each $\varphi_{i}$ has a boundary map $\varphi_{i}^{*}: \partial \mathbb{B}^{n} \rightarrow \overline{\mathbb{B}}_{p(\sigma), q(\sigma)}$ defined almost everywhere on $\partial \mathbb{B}^{n}$ and given by $\varphi_{i}^{*}(x)=\lim _{n \rightarrow \infty} \varphi_{i}\left(\xi_{n}\right)$ for any sequence of points $\xi_{n} \rightarrow x$ that stay in an angular sector $D_{\alpha}(x)$.

Since $\Gamma$ is a lattice, it is in particular a discrete subgroup of divergence type, and hence the set points of approximation in $\partial \mathbb{B}^{n}$ (those that can be approximated by a sequence in $\Gamma \cdot z$ that stays in a angular sector $\left.D_{\alpha}\right)$ is of full Lebesgue measure. Consequently, we obtain $\varphi_{i}^{*}(x)=\lim \varphi\left(\gamma_{n}\left(x_{0}\right)\right)$ for some $x_{0} \in \mathbb{B}^{n}$ and a sequence of $\gamma_{n} \in \Gamma$. We may replace $x_{0}$ by any other point $y_{0} \in \mathbb{B}^{n}$ and still $x=\lim \gamma_{n}\left(y_{0}\right)$, since the hyperbolic distances $d\left(\gamma_{n}\left(y_{0}\right), \gamma_{n}\left(x_{0}\right)\right)=d\left(x_{0}, y_{0}\right)$ are unchanged.

We claim that the boundary maps agree, i.e. $\varphi_{1}^{*}=\varphi_{2}^{*}$ on $\partial \mathbb{B}^{n}$. For this purpose we first show that almost everywhere $\varphi_{i}^{*}$ maps $\partial \mathbb{B}^{n}$ to $\partial \mathbb{B}_{p(\sigma), q(\sigma)}$ (compare to Shi04, Lemma 2.2]). Since $\varphi_{i}$ is non-constant, there exist $x_{0}, y_{0} \in \mathbb{B}^{n}$ with $\varphi_{i}\left(x_{0}\right) \neq \varphi_{i}\left(y_{0}\right)$. This implies

$$
0<d\left(\varphi_{i}\left(x_{0}\right), \varphi_{i}\left(y_{0}\right)\right)=d\left(\gamma_{n}^{\sigma}\left(\varphi_{i}\left(x_{0}\right)\right), \gamma_{n}^{\sigma}\left(\varphi_{i}\left(y_{0}\right)\right)\right)=d\left(\varphi_{i}\left(\gamma_{n}\left(x_{0}\right)\right), \varphi_{i}\left(\gamma_{n}\left(y_{0}\right)\right)\right) .
$$

If $x=\lim \gamma_{n}\left(x_{0}\right) \in \partial \mathbb{B}^{n}$ is a point of approximation, taking the limit $n \rightarrow \infty$ the inequality gives a contradiction if $\varphi_{i}^{*}(x)$ lies in the interior of $\mathbb{B}_{p(\sigma), q(\sigma)}$. This argument also shows that for any such approximation $x=\lim \gamma_{n}\left(x_{0}\right)$ the sequence $\gamma_{n}^{\sigma}\left(\varphi_{i}\left(x_{0}\right)\right)$ goes to the boundary of $\mathbb{B}_{p(\sigma), q(\sigma)}$. The limit point of such a sequence is known to exist since $\varphi_{i}^{*}$ is well-defined and, by the same argument as above in $\mathbb{B}^{n}$, it only depends on the group elements $\gamma_{n}^{\sigma}$, not on the starting point. Consequently,

$$
\varphi_{1}^{*}(x)=\lim \varphi_{1}\left(\gamma_{n}\left(x_{0}\right)\right)=\lim \gamma_{n}^{\sigma}\left(\varphi_{1}\left(x_{0}\right)\right)=\lim \gamma_{n}^{\sigma}\left(\varphi_{2}\left(x_{0}\right)\right)=\varphi_{2}^{*}(x),
$$

as we claimed. 
Now, the Cauchy integral formula is still valid for these boundary maps ( $\operatorname{Rud} 87$ Theorem 11.32 and Theorem 17.18]), since we may also use radial limits to define $\varphi_{i}^{*}$. These theorems imply that the maps $\varphi_{1}$ and $\varphi_{2}$ agree.

For the proof of the second statement we may pass to a subgroup of finite index and thus we suppose that $\Gamma$ itself satisfies the conclusion of Lemma 4.2. By this assumption and definition $\tau$ fixes all traces of elements in $\Gamma$ and consequently the two representations $\Gamma^{\sigma \tau}$ and $\Gamma^{\sigma}$ are isomorphic (e.g. [Lan02, Corollary XVII.3.8]), in particular their signatures agree. If $M$ is the matrix that provides the isomorphism, i.e. $M \gamma^{\sigma} M^{-1}=\gamma^{\sigma \tau}$, then both $\varphi_{2}$ and $M \circ \varphi_{1}$ are equivariant with respect to $\Gamma$ and $\Gamma^{\sigma \tau}$. By the first part these two maps agree.

In the situation considered everywhere in the sequel, the existence of a modular embedding is no obstruction thanks to the following proposition, but in general the existence of modular embeddings is a hard problem, even for one-dimensional ball quotients, i.e. for Fuchsian groups.

Proposition 4.4. $\Gamma$ arises as the monodromy group of a uniformizing sub-local system $\mathbb{W}$ in a polarized $\mathbb{Q}-V H S \mathbb{V}$ of weight 1 if and only if $\Gamma$ admits a modular embedding.

Proof. Suppose that $\Gamma$ is the monodromy group of a uniformizing sub-local system of $\mathbb{V}$. By Deligne's semisimplicity theorem, $\mathbb{V}_{\mathbb{C}}$ decomposes into a direct sum of irreducible $\mathbb{C}$-VHS, one of which is $\mathbb{W}$. Since $\mathbb{V}$ is defined over $\mathbb{Q}$, all Galois conjugate local systems $\mathbb{W}^{\sigma}$ appear in the decomposition of $\mathbb{V}_{\mathbb{C}}$. After tensoring with $\mathbb{C}$, these $\mathbb{W}^{\sigma}$ are polarized $\mathbb{C}$-VHS of weight 1 , thus their monodromy representation fixes an indefinite hermitian form of some signature $(p(\sigma), q(\sigma))$, and their period map $\varphi^{\sigma}$ is an equivariant map $\mathbb{B}^{n} \rightarrow \mathbb{B}_{p(\sigma), q(\sigma)}$ as desired.

Conversely, let $\Gamma$ have a modular embedding. Each group homomorphism $\Gamma \rightarrow$ $\Gamma^{\sigma}$ determines a $\mathbb{C}$-local system $\mathbb{W}^{\sigma}$. These carry a polarized VHS of weight 1 induced by the map $\varphi^{\sigma}$. The direct sum $\bigoplus_{\sigma \in \operatorname{Gal}(F / \mathbb{Q})} \mathbb{W}^{\sigma}$ is a polarized VHS that is defined over $\mathbb{Q}$.

4.2. Commensurability invariants. Fix a lattice $\Gamma \subset \mathrm{SU}(1, n) \cap \mathrm{GL}_{n+1}(F)$ with modular embedding. This determines a Hodge structure on the corresponding local system $\mathbb{W}^{\sigma}$ on the ball quotient $B=\mathbb{B}^{n} / \Gamma$. We focus on the case of signature $(1, n)$. The subbundle of holomorphic forms $\mathcal{E}^{1,0}$ is thus of rank one. All the numbers $\left(\mathrm{c}_{1}\left(\mathcal{E}^{1,0}\right)^{a} \cdot \mathrm{c}_{1}\left(\omega_{\bar{B}}\right)^{b}\right) / \mathrm{c}_{1}\left(\omega_{\bar{B}}\right)^{n}$ for $(a, b) \in \mathbb{N}_{0}^{2}$ with $a+b=n$ are invariants of the modular embedding. By homogeneity, they are also unchanged under passage from $\Gamma$ to a subgroup of finite index. By the preceding Theorem 4.3 there is no choice involved in fixing the modular embedding, and these numbers only depend on the coset of $\sigma$ in $\operatorname{Gal}(F / \mathbb{Q}) / \operatorname{Gal}(F / E)$. Altogether, this yields the following.

Corollary 4.5. For any $(a, b) \in \mathbb{N}_{0}^{2}$ with $a+b=n$ and $\sigma \in \operatorname{Gal}(F / \mathbb{Q}) / \operatorname{Gal}(F / E)$ the ratios

$$
\frac{\mathrm{c}_{1}\left(\mathcal{E}^{1,0}\right)^{a} \cdot \mathrm{c}_{1}\left(\omega_{\bar{B}}\right)^{b}}{\mathrm{c}_{1}\left(\omega_{\bar{B}}\right)^{n}}, \quad \mathcal{E}^{1,0}=\mathcal{E}^{1,0}\left(\mathbb{W}^{\sigma}\right)
$$

are commensurability invariants of $\Gamma$.

We end this section with a proof of this corollary for the special case $a=1$, i.e. for Lyapunov exponents, not relying on Theorem 4.3. but rather on the definition of Lyapunov exponents. 
Proposition 4.6. In the situation of Theorem [1.1, passing from $\Gamma$ to a subgroup $\Gamma^{\prime}$ of finite index and to the pullback VHS does not change the Lyapunov spectrum.

Proof. Let $\mathcal{F} \subset \mathbb{B}$ be a fundamental domain for $\Gamma$ and $\mathcal{F}^{\prime} \supset \mathcal{F}$ be a fundamental domain for $\Gamma^{\prime}$. Given a starting point $x \in M=T_{1} \mathbb{B}^{n}$, for all times $t$ such that $g_{t}(x)$ is $\Gamma^{\prime}$-equivalent to a point in $\mathcal{F}$, the quantity $\frac{1}{t} \log \left\|g_{t}(v)\right\|$ appearing on the right of (15) is the same for both situations. Since the geodesic flow is ergodic, this set of times is cofinal and thus the limit in (15) is the same in both situations.

Definition 4.7. Let $\Gamma$ be a lattice admitting a modular embedding, and let $\mathbb{W}$ be the uniformizing VHS on $\mathbb{B}^{n} / \Gamma$. The Lyapunov spectrum of $\Gamma$ is the set of different Lyapunov exponents in the Lyapunov spectrum of the $\mathbb{Q}$-VHS

$$
\mathbb{P}(\Gamma)=\bigoplus_{\sigma \in \operatorname{Gal}(F / \mathbb{Q})} \mathbb{W}^{\sigma} .
$$

The definition might a priori depend on the choice of the modular embedding. We show that this is not the case.

Theorem 4.8. The Lyapunov spectrum of a lattice $\Gamma$ in $\mathrm{PU}(1, n)$ that admits a modular embedding is well-defined. It is a commensurability invariant among all lattices in $\mathrm{PU}(1, n)$ that admit a modular embedding.

Proof. The Lyapunov spectrum is determined by the choice of the geodesic flow on the base $B$, the Haar measure $\mu$ on $B$, the local systems with monodromy group $\Gamma^{\sigma}$ and a norm satisfying the growth conditions in Theorem 2.5. Any choice of a modular embedding defines a Hodge structure and thus a corresponding Hodge norm. But the Lyapunov spectrum does not depend on this choice by Lemma 2.6.

Note that for a lattice $\Gamma$ admitting a modular embedding, the $\mathbb{Q}$-VHS $\mathbb{P}(\Gamma)$ is equal to its primitive part. If on the other hand, $\mathbb{V}$ is a $\mathbb{Q}$-VHS on $\mathbb{B}^{n} / \Gamma$ containing a primitive part $\mathbb{P}$, then the uniqueness of the uniformizing sub-VHS and the above theorem yield that the set of Lyapunov exponents of $\mathbb{P}$ (forgetting multiplicities) is equal to the Lyapunov spectrum of $\Gamma$.

\section{Arithmeticity}

Recall that a lattice $\Gamma \subset \mathrm{PU}(1, n)$ is called arithmetic, if $\Gamma$ is commensurable to $\phi\left(G_{\mathbb{Z}}\right)$, the image of the integral points in some linear algebraic group $G_{\mathbb{R}}$, admitting a continuous surjective homomorphism $\phi: G_{\mathbb{R}} \rightarrow \mathrm{PU}(1, n)$ with compact kernel.

Let $\mathbb{P}$ be a $\mathbb{Q}$-irreducible $\mathbb{Q}$-VHS. Note that a uniformizing $\mathbb{C}$-sub-VHS $\mathbb{W}$ of signature $(1, n)$ of $\mathbb{P}_{\mathbb{C}}$ is defined over $\mathbb{R}$ if and only if $n=1$. We say that the Lyapunov spectrum of $\mathbb{P}$ is maximally degenerate, if it contains twice the Lyapunov exponents 1 and -1 for $n>1$ (resp. once for $n=1$ ) and all the other Lyapunov exponents are zero.

Proposition 5.1. If $\Gamma \subset \mathrm{PU}(1, n)$ is an arithmetic lattice, then the Lyapunov spectrum is maximally degenerate.

Proof. Suppose that $\Gamma$ is arithmetic. Then $\mathbb{W}$ (and hence $\overline{\mathbb{W}}$ ) is uniformizing, and all other conjugates of $\mathbb{W}$ are unitary ([DM86, 12.2.6]). In particular, $\Gamma$ admits a modular embedding with $\varphi^{\sigma}$ being either the identity or constant. For all conjugates of $\mathbb{W}$ the Lyapunov spectrum is determined by Theorem $1.1 \mathrm{i}$ ) and iii) and this is precisely the content of the notion maximally degenerate. 
We remark that the converse of the preceding proposition is an interesting problem. Suppose that some $\mathbb{Q}$-irreducible VHS $\mathbb{P}$ over some ball quotient $B=\mathbb{B}^{n} / \Gamma$ is maximally degenerate. If we knew that $\mathbb{P}_{\mathbb{C}}$ contained a sub-VHS $\mathbb{W}$ of signature $(1, n)$, then maximally degenerate implies that this $\mathbb{W}$ reaches the Arakelov bound by (2). Then $\mathbb{W} \oplus \overline{\mathbb{W}}$ uses up all the non-zero Lyapunov exponents. In the complement, the norm growth along the geodesic flow is subexponential. By the argument in [Möl11, Proposition 5.4] this complement is in fact a unitary VHS. Consequently, we can conclude as in VZ07 or MVZ12 to show that $\Gamma$ is arithmetic. The missing step is thus the question whether there exists at all such a $\mathbb{C}$-sub-VHS of $\mathbb{P}_{\mathbb{C}}$.

\section{CyClic COVers}

The section contains well-known general material that is used as preparation for the next section. We start with generalities on cyclic coverings and then recall which cyclic coverings give rise to a structure of a ball quotient on the parameter space.

6.1. The parameter space for cyclic coverings. Let $N \geq 3$ and let $B_{0}=\mathcal{M}_{0, N}$ denote the moduli space (or configuration space) of $N$ ordered points on $\mathbb{P}^{1}=\mathbb{P}^{1}(\mathbb{C})$. Explicitly, if we let

$$
M_{0}=\left\{x=\left(x_{1}, \ldots, x_{N}\right) \in\left(\mathbb{P}^{1}\right)^{N} \mid \forall i, j \in\{1, \ldots, N\}: x_{i} \neq x_{j}\right\}
$$

then $\mathrm{PGL}_{2}(\mathbb{C})$ acts diagonally on $M_{0}$ and

$$
B_{0}=\mathrm{PGL}_{2}(\mathbb{C}) \backslash M_{0} .
$$

Let $a, b, c \in \mathbb{P}^{1}$ be distinct and consider the space of normalized tuples

$$
\left\{x \in M_{0} \mid x_{1}=a, x_{N-1}=b, x_{N}=c\right\} .
$$

We may identify $B_{0}$ with this set. A point in $B_{0}$ will be denoted by $\underline{x}$; we will often choose a convenient representative by fixing three coordinates.

6.1.1. The type of a cyclic covering. A tuple $\left(d ; a_{1}, \ldots, a_{N}\right)$ of natural numbers is called a type of a cyclic covering if

$$
d \geq 2, \quad 0<a_{i}<d, \quad \operatorname{gcd}\left(a_{1}, \ldots, a_{N}, d\right)=1, \quad \sum_{i=1}^{N} a_{i} \in d \mathbb{Z} .
$$

A cyclic covering of type $\left(d ; a_{1}, \ldots, a_{N}\right)$ branched at $\underline{x} \in B_{0}$ is the complete, nonsingular curve $X$ with affine equation

$$
y^{d}=\prod_{i=1}^{N}\left(x-x_{i}\right)^{a_{i}}
$$

Its genus is given by the Riemann-Hurwitz formula

$$
g(X)=\frac{N-2}{2} d+1-\frac{1}{2} \sum_{i=1}^{N} \operatorname{gcd}\left(a_{i}, d\right)
$$

By varying $\underline{x} \in B_{0}$ we obtain a family of cyclic covers of the same type

$$
f: \mathcal{X} \rightarrow B_{0} \text {. }
$$

Since this family is locally topologically trivial, the sheaf $R^{1} f_{*}(\mathbb{C})$ is a local system on $B_{0}$. 
6.1.2. Stable and semi-stable points, cusps. We enlarge $B_{0}$ by adding divisors in the boundary that parametrize stable points (compare [DM86, Section 4]) and this enlarged space will have the structure of a ball quotient. Sometimes the ball quotient is not yet compact and we add some boundary points, called cusps, that parametrize classes of semi-stable points in $M$.

For a fixed $\mu=\left(\mu_{i}\right)_{1 \leq i \leq N} \in(\mathbb{Q} \cap(0,1))^{N}$ such that $\sum_{i} \mu_{i}=2$, we call $x \in\left(\mathbb{P}^{1}\right)^{N}$ a $\mu$-stable (resp. $\mu$-semi-stable) point, if for all $z \in \mathbb{P}^{1}$

$$
\sum_{i: x_{i}=z} \mu_{i}<1 \quad\left(\text { resp. } \sum_{i: x_{i}=z} \mu_{i} \leq 1\right) .
$$

Define $M=M^{\mu} \supset M_{0}$ to be the set of $\mu$-stable points and let

$$
B=B^{\mu}=\mathrm{PGL}_{2}(\mathbb{C}) \backslash M .
$$

Then $B$ is a complex manifold containing $B_{0}$ as an open subspace. We use throughout the convention adopted here to refer to the tuple of weights defining (semi)stable points by upper indices (like $B^{\mu}$ or $B^{k}$ later), while lower indices refer to parameters of the local system (or period maps) on these spaces.

Next let $\bar{M}=\bar{M}^{\mu} \supset M^{\mu}$ be the set of $\mu$-semi-stable points. If $x \in\left(\mathbb{P}^{1}\right)^{N}$ is strictly semi-stable, then there is a unique partition of $\{1, \ldots, N\}=S_{1} \dot{\cup} S_{2}$, such that $\sum_{k \in S_{j}} \mu_{k}=1$ for $k=1,2, x_{i} \neq x_{j}$ for $i \in S_{1}, j \in S_{2}$, and $i \mapsto x_{i}$ is constant on $S_{1}$ or on $S_{2}$. To define $\bar{B}$, we first take the quotient of $\bar{M}$ by $\mathrm{PGL}_{2}(\mathbb{C})$ and then identify the classes of strictly semi-stable points having the same partition. The elements of $\bar{B} \backslash B$ are called cusps. $\bar{B}$ with its quotient topology is a compact Hausdorff space and an algebraic variety [DM86, 4.3, 4.4.2, 4.5].

Let $\{i, j\} \subset\{1, \ldots, N\}$ be a two-element subset such that $\mu_{i}+\mu_{j}<1$. Define the elliptic divisor $L_{i j}=L_{i j}^{\mu}$ as the image of

$$
\left\{x=\left(x_{1}, \ldots, x_{N}\right) \in M \mid x_{i}=x_{j}\right\}
$$

under the projection modulo $\mathrm{PGL}_{2}(\mathbb{C})$. The interior $L_{i j}^{\circ}$ of $L_{i j}$ is defined as the subset of $L_{i j}$ where precisely two coordinates are equal.

6.2. Cyclic coverings uniformized by the ball. For a cyclic covering of type $\left(d ; a_{1}, \ldots, a_{N}\right)$, define $\mu_{i}=a_{i} / d$. The following condition on the $\mu_{i}$ was the first criterion that provided a general construction of ball quotients.

(INT) $\sum_{i=1}^{N} \mu_{i}=2, \quad$ and $\quad$ for all $i \neq j$ with $\mu_{i}+\mu_{j}<1,\left(1-\mu_{i}-\mu_{j}\right)^{-1} \in \mathbb{Z}$

Theorem 6.1 (DM86]). If $\mu$ satisfies (INT) then $B$ is a ball quotient.

One can relax the condition (INT) in order to be more flexible and to obtain more

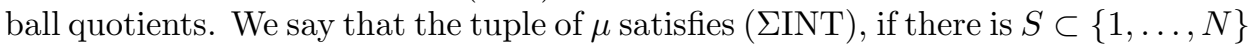
such that the following conditions hold.

$$
\sum_{i=1}^{N} \mu_{i}=2, \quad \text { and } \quad \mu_{i}=\mu_{j} \quad \text { for all } \quad i, j \in S \quad \text { and moreover }
$$

$$
\text { for } i \neq j \text { with } \mu_{i}+\mu_{j}<1:\left(1-\mu_{i}-\mu_{j}\right)^{-1} \in \begin{cases}\frac{1}{2} \mathbb{Z} & i, j \in S \\ \mathbb{Z} & \text { otherwise }\end{cases}
$$


Let $\Sigma=\operatorname{Sym}(S)$ act on $B$ by permuting coordinates.

Theorem 6.2 (Mos86). If $\mu$ satisfies (INT) then $B / \Sigma$ is a ball quotient.

6.3. The non-arithmetic examples. In the following table we reproduce (and number) the non-arithmetic examples of two-dimensional ball quotients of [DM86] and $\operatorname{Mos} 86]$, according to the corrected table in $\operatorname{Mos} 88$. Note that example no. 5 in loc. cit. has a misprint concerning the $a_{i}$.

\begin{tabular}{|c|c|c|c|c|c|c|c|c|c|c|c|}
\hline & $d$ & $a_{1}$ & $a_{2}$ & $a_{3}$ & $a_{4}$ & $a_{5}$ & & & $L_{i j}^{\mathrm{par}}$ & $g$ & comm. to \\
\hline 1 & 12 & 3 & 3 & 3 & 7 & 8 & $I N T$ & $B_{9}$ & -- & 12 & 4 \\
\hline 2 & 12 & 3 & 3 & 5 & 6 & 7 & $I N T$ & $B_{9}$ & $L_{35}$ & 12 & \\
\hline 3 & 12 & 4 & 4 & 4 & 5 & 7 & $I N T$ & $B_{10}$ & $L_{45}$ & 12 & \\
\hline 4 & 12 & 4 & 4 & 5 & 5 & 6 & $I N T$ & $B_{10}$ & -- & 11 & 1 \\
\hline 5 & 15 & 4 & 6 & 6 & 6 & 8 & $I N T$ & $B_{10}$ & -- & 18 & 13 \\
\hline 6 & 18 & 2 & 7 & 7 & 7 & 13 & $\Sigma I N T$ & $B_{7} / \Sigma_{3}$ & -- & 25 & 7 \\
\hline 7 & 18 & 7 & 7 & 7 & 7 & 8 & $\Sigma I N T$ & $B_{10} / \Sigma_{4}$ & -- & 25 & 6 \\
\hline 8 & 20 & 5 & 5 & 5 & 11 & 14 & $I N T$ & $B_{9}$ & -- & 22 & 9 \\
\hline 9 & 20 & 6 & 6 & 9 & 9 & 10 & $\Sigma I N T$ & $B_{10} / \Sigma_{2}$ & -- & 23 & 8 \\
\hline 10 & 20 & 6 & 6 & 6 & 9 & 13 & $\Sigma I N T$ & $B_{9} / \Sigma_{3}$ & -- & 27 & \\
\hline 11 & 24 & 4 & 4 & 4 & 17 & 19 & $\Sigma I N T$ & $B_{9} / \Sigma_{3}$ & -- & 30 & 12 \\
\hline 12 & 24 & 7 & 9 & 9 & 9 & 14 & $I N T$ & $B_{10}$ & -- & 31 & 11 \\
\hline 13 & 30 & 5 & 5 & 5 & 22 & 23 & $\Sigma I N T$ & $B_{9} / \Sigma_{3}$ & -- & 37 & 5 \\
\hline 14 & 42 & 7 & 7 & 7 & 29 & 34 & $\Sigma I N T$ & $B_{9} / \Sigma_{3}$ & -- & 53 & 15 \\
\hline 15 & 42 & 13 & 15 & 15 & 15 & 26 & $\Sigma I N T$ & $B_{10} / \Sigma_{3}$ & -- & 58 & 14 \\
\hline
\end{tabular}

The trace fields of these lattices are $\mathbb{Q}[\cos (2 \pi / d)]$. They are distinct if and only if the $d$ are distinct with the exception that $d=15$ and $d=30$ produce the same trace field (and indeed commensurable lattices). The commensurability results of Sauter and Deligne-Mostow (see [Par09, Section 3]) among these lattices are indicated in the last column.

In dimension three, there is a unique commensurability class of a non-arithmetic ball quotient known, given by the cyclic covering of type $(12 ; 7,5,3,3,3,3)$.

6.4. Realizations of the compactification and their intersection rings. For technical reasons we will need the boundary of the ball quotients to be a normal crossing divisor. By DM86, Lemma 4.5.1], $\bar{B}$ is smooth if for all cusps, the associated partition $\left\{S_{1}, S_{2}\right\}$ satisfies $\left|S_{1}\right|=2$ or $\left|S_{2}\right|=2$. Fortunately, this applies to all cases that we need to consider. We will thus blow up the cusps and in the blowup $B^{\text {nc }} \rightarrow \bar{B}$ the preimage of the cusp is now a divisor, called boundary divisor. In the two-dimensional case, each of these divisors can be identified with the image modulo $\mathrm{PGL}_{2}(\mathbb{C})$ of the set of all semi-stable points in $M$ with $x_{i}=x_{j}$ for some $i, j$. We will write $L_{i j}^{\text {par }}$ to distinguish these divisor from elliptic divisors which are also some $L_{i j}$, but the monodromy is elliptic, as opposed to parabolic in the case of boundary divisors.

6.4.1. Dimension two, case (INT). We start with two-dimensional ball quotients satisfying (INT). In all the cases that we will be interested in $B^{\text {nc }}$ will contain all the 10 divisors $L_{i j}$ (some of them possibly being $L_{i j}^{\text {par }}$ ) or 9 out of the 10 . 
We call the first case $B_{10}$. In this case $\left|L_{i j} \cap L_{k l}\right|=1$ if $\{i, j\} \cap\{k, l\}=\emptyset$ and $L_{i j} \cap L_{k l}=\emptyset$ other wise. The variety $B_{10}$ is isomorphic to $\mathbb{P}^{2}$ blown up at 4 points in general position [Yos87, §10.5]. Therefore, $\mathrm{CH}_{1}\left(B_{10}\right)$ is generated by

$$
h, e_{1}, \ldots, e_{4}
$$

where $h$ is the pullback of the hyperplane class in $\mathrm{CH}_{1}\left(\mathbb{P}^{2}\right)$, and $e_{1}, \ldots, e_{4}$ are the classes of the four exceptional divisors. The ten lines $L_{i j}$ are given by the strict transforms of the six lines in $\mathbb{P}^{2}$ connecting the four points, and by the four exceptional divisors. The classes of the lines can be expressed in the generators (up to renumbering) as

$$
\begin{aligned}
& {\left[L_{i j}\right]=h-e_{k}-e_{m} \quad \text { where } \quad\{i, j, k, m\}=\{1, \ldots, 4\}} \\
& {\left[L_{i 5}\right]=e_{i} .}
\end{aligned}
$$

The intersection matrix with respect to the ordering $h, e_{1}, e_{2}, e_{3}, e_{4}$ is

$$
\operatorname{diag}(1,-1,-1,-1,-1),
$$

and the canonical class is $K_{B_{10}}=-3 h+e_{1}+e_{2}+e_{3}+e_{4}$.

We call the second case $B_{9}$ and we may choose indices so that $B_{10} \rightarrow B_{9}$ contracts $L_{45}$. Said differently, $B_{9}$ can be obtained by blowing up $\mathbb{P}^{2}$ at three points in general position. Now, $\mathrm{CH}_{1}\left(B_{9}\right)=\left\langle h, e_{1}, e_{2}, e_{3}\right\rangle$ and

$$
\begin{aligned}
& {\left[L_{12}\right]=h-e_{3}, \quad\left[L_{13}\right]=h-e_{2}, \quad\left[L_{23}\right]=h-e_{1}} \\
& {\left[L_{i 4}\right]=h-e_{k}-e_{m} \quad \text { where } \quad\{i, k, m\}=\{1,2,3\}} \\
& {\left[L_{i 5}\right]=e_{i} .}
\end{aligned}
$$

The intersection pairing has matrix $\operatorname{diag}(1,-1,-1,-1)$ and the canonical class is $K_{B_{9}}=-3 h+e_{1}+e_{2}+e_{3}$.

6.4.2. Dimension two, case ( $\Sigma$ INT). We will also need to work with the quotient of $B$ by a symmetric group $\Sigma$. Let $S \subseteq\{1, \ldots, 5\}$, and let $\Sigma=\operatorname{Sym}(S)$ act on $B$ by permuting the coordinates. The Chow ring $\mathrm{CH}_{*}(B / \Sigma)_{\mathbb{Q}}$ is isomorphic to $\left(\mathrm{CH}_{*}(B)_{\mathbb{Q}}\right)^{\Sigma}$, and the ring structure is given by

$$
\left(D_{1} \cdot D_{2}\right)=1 /|\Sigma| \cdot \eta_{*}\left(\eta^{*} D_{1} \cdot \eta^{*} D_{2}\right)
$$

where $\eta: B \rightarrow B / \Sigma$ denotes the quotient map. Note that the pullback of a divisor is the sum of the irreducible components of the preimage, each weighted with the order of its stabilizer. Moreover, by [Ful84, 17.4.10], the ring structure is independent of the presentation of $B / \Sigma$ as a quotient by a finite group.

We only present the cases of $B$ and $\Sigma$ that are actually needed in the subsequent computations. The case of a permutation group of 4 elements appearing in line 7 of the above table will not be treated, since it is covered by the case in line 6 and the commensurability Theorem 4.8

The case $B_{9} / \Sigma_{3}$. Let $S=\{1,2,3\}$, and consider the action of $\Sigma_{3}=\operatorname{Sym}(S)$ on $B=B_{9}$. The map $\eta$ is ramified of order 2 along the divisors $L_{12}, L_{23}$ and $L_{13}$. The group $\mathrm{CH}_{1}\left(B_{9} / \Sigma_{3}\right)_{\mathbb{Q}}$ is generated by the images $\left[\bar{L}_{14}\right]$, $\left[\bar{L}_{15}\right]$, and $\left[\bar{L}_{12}\right]$ of the 
divisors and

$$
\begin{aligned}
\eta^{*}\left[\bar{L}_{14}\right] & =\left[L_{14}\right]+\left[L_{24}\right]+\left[L_{34}\right], \\
\eta^{*}\left[\bar{L}_{15}\right] & =\left[L_{15}\right]+\left[L_{25}\right]+\left[L_{35}\right], \\
\eta^{*}\left[\bar{L}_{12}\right] & =2\left(\left[L_{12}\right]+\left[L_{23}\right]+\left[L_{13}\right]\right) .
\end{aligned}
$$

Note that $\left[\bar{L}_{12}\right]=2\left(\left[\bar{L}_{14}\right]+\left[\bar{L}_{15}\right]\right)$. The intersection matrix with respect to this system of generators is

$$
\left(\begin{array}{ccc}
-1 / 2 & 1 & 1 \\
1 & -1 / 2 & 1 \\
1 & 1 & 4
\end{array}\right)
$$

By Riemann-Hurwitz,

$$
\eta^{*} K_{B_{9} / \Sigma_{3}}=K_{B_{9}}-\left(\left[L_{12}\right]+\left[L_{23}\right]+\left[L_{13}\right]\right) .
$$

By intersecting with all generators in $\mathrm{CH}_{1}\left(B_{9} / \Sigma_{3}\right)_{\mathbb{Q}}$, we obtain

$$
K_{B_{9} / \Sigma_{3}}=-\left[\bar{L}_{12}\right] \text {. }
$$

The case $B_{7} / \Sigma_{3}$. Again, let $S=\{1,2,3\}$, and let $B_{7}$ be the space obtained from $B_{10}$ by collapsing all $L_{i 5}, i=1, \ldots, 3$. This is the projective plane blown up at one point. Let again $h$ denote the hyperplane class and let $e$ denote the class of the exceptional divisor. Then the classes of the boundary divisors are given by

$$
\begin{aligned}
& {\left[L_{i 4}\right]=h, \quad i=1,2,3} \\
& {\left[L_{12}\right]=\left[L_{23}\right]=\left[L_{13}\right]=h-e} \\
& {\left[L_{45}\right]=e}
\end{aligned}
$$

The Chow group $\mathrm{CH}_{1}\left(B_{7} / \Sigma_{3}\right)_{\mathbb{Q}}$ is generated by $\left[\bar{L}_{12}\right],\left[\bar{L}_{14}\right]$ and $\left[\bar{L}_{45}\right]$, and

$\eta^{*}\left[\bar{L}_{12}\right]=2\left(\left[L_{12}\right]+\left[L_{23}\right]+\left[L_{13}\right]\right), \quad \eta^{*}\left[\bar{L}_{14}\right]=\left[L_{14}\right]+\left[L_{24}\right]+\left[L_{34}\right], \quad \eta^{*}\left[\bar{L}_{45}\right]=\left[L_{45}\right]$.

The respective matrix of the intersection product is

$$
\left(\begin{array}{ccc}
0 & 3 & 1 \\
3 & 3 / 2 & 0 \\
1 & 0 & -1 / 6
\end{array}\right),
$$

and the canonical class is given by

$$
K_{B_{7} / \Sigma_{3}}=-\frac{2}{3}\left(\left[\bar{L}_{12}\right]+\left[\bar{L}_{14}\right]\right) .
$$

6.4.3. Dimension three. In order to compute the intersection ring of the moduli space $B_{14}$ (we keep indexing by the number of boundary divisors of stable configurations) of the three-dimensional ball quotient we use the formalism of weighted stable curves of Hassett ([Has03]). In his language, we are interested in $\overline{\mathcal{M}}_{0 ;\left(\frac{7}{12}, \frac{5}{12}, \frac{1}{4}, \frac{1}{4}, \frac{1}{4}, \frac{1}{4}\right)}$. The space $\overline{\mathcal{M}}_{0 ;\left(1, \frac{1}{4}, \frac{1}{4}, \frac{1}{4}, \frac{1}{4}, \frac{1}{4}\right)}$ is isomorphic to $\mathbb{P}^{3}$ and $\overline{\mathcal{M}}_{0 ;\left(1, \frac{2}{4}, \frac{1}{4}, \frac{1}{4}, \frac{1}{4}, \frac{1}{4}\right)}$ is the blowup of $\mathbb{P}^{3}$ corresponding to curves where the points with labels $I_{3}=\{2,4,5,6\}, I_{4}=$ $\{2,3,5,6\}, I_{5}=\{2,3,4,6\}$ resp. $I_{6}=\{2,3,4,5\}$ come together. Reducing the weights from $\left(1, \frac{2}{4}, \frac{1}{4}, \frac{1}{4}, \frac{1}{4}, \frac{1}{4}\right)$ to $\left(1, \frac{5}{12}, \frac{1}{4}, \frac{1}{4}, \frac{1}{4}, \frac{1}{4}\right)$ and further to $\left(\frac{7}{12}, \frac{5}{12}, \frac{1}{4}, \frac{1}{4}, \frac{1}{4}, \frac{1}{4}\right)$ is an isomorphism, as can be checked using the criterion in [Has03, Section 4.2]. Consequently, we are interested in a projective space $\mathbb{P}^{3}$, blown up at four points $P_{i}$ corresponding to the set of indices $I_{i}, i=3,4,5,6$. 
We deduce from [Ful84, Proposition 6.7 (e)] that Pic $\left(B_{14}\right)$ is freely generated by the pullback $h$ of the hyperplane class of $\mathbb{P}^{3}$ and the classes of the exceptional divisors $e_{3}, e_{4}, e_{5}, e_{6}$. Moreover, $\mathrm{CH}_{1}\left(B_{14}\right)$ is generated by the pullback of a line $\ell$ from $\mathbb{P}^{3}$ and lines $\ell_{i}$ on the exceptional divisors. The ring structure of the chow ring is given by $h \cdot h=\ell, e_{i} \cdot e_{i}=(-1) \cdot \ell_{1}$ and $h \cdot \ell=1, e_{i} \cdot l_{i}=1$ as well as zero for all intersections not listed above.

If we normalize $x_{1}=\infty$ and let $z_{i}=x_{2}-x_{i}$, then $\left(z_{3}: z_{4}: z_{5}: z_{6}\right)$ is indeed a coordinate system, in which $P_{3}=(1: 0: 0: 0)$ etc. and the semi-stable point is $P_{12}=(1: 1: 1: 1)$. In these coordinates, one checks that

$$
\begin{aligned}
& L_{1 j}=e_{j}, \quad L_{2 j}=h-e_{3}-e_{4}-e_{5}-e_{6}+e_{j}, \quad j=3,4,5,6 . \\
& L_{j k}=h-e_{3}-e_{4}-e_{5}-e_{6}+e_{j}+e_{j} \quad 3 \leq j<k \leq 6
\end{aligned}
$$

Finally, the formula for the behaviour of the canonical class yields

$$
K_{B_{14}}=-4 h+2 e_{3}+2 e_{4}+2 e_{5}+2 e_{6} .
$$

6.5. Decomposition of the VHS for cyclic coverings. A general reference for the following discussion is Bou05 and Bou01. Let $G=\operatorname{Gal}\left(X / \mathbb{P}^{1}\right) \cong \mathbb{Z} /(d)$ be the Galois group of a cyclic cover of type $\left(d ; a_{1}, \ldots, a_{N}\right)$. Fix a primitive $d$-th root of unity $\zeta$, the generator $g$ of $G$ given by

$$
g: x \mapsto x, \quad y \mapsto \zeta y
$$

and let $\chi: G \rightarrow \mathbb{C}^{\times}$be the character of $G$ defined by $\chi(g)=\zeta^{-1}$. As $G$ acts on $H_{\mathrm{dR}}^{1}(X)$, we can decompose this space into eigenspaces

$$
H_{\mathrm{dR}}^{1}(X)=\bigoplus_{k=1}^{d} H_{\mathrm{dR}}^{1}(X)_{\chi^{k}} .
$$

Since the action of $G$ respects the Hodge decomposition $H_{\mathrm{dR}}^{1}(X)=H^{1,0} \oplus H^{0,1}$, we also have a decomposition of $H^{1,0}$ and $H^{0,1}$.

The eigenspace decomposition carries over to the relative situation of (18). We have

$$
R^{1} f_{*} \mathbb{C}=\bigoplus_{k=1}^{d} \mathbb{L}_{k} .
$$

Let

$$
\mathcal{L}_{k}^{1,0} \subseteq \mathbb{L}_{k} \otimes \mathcal{O}_{B_{0}}
$$

be the subbundle of holomorphic forms, and let

$$
\mathcal{L}_{k}^{0,1}=\left(\mathbb{L}_{k} \otimes \mathcal{O}_{B_{0}}\right) / \mathcal{L}_{k}^{1,0}
$$

The Chevalley-Weil formula gives information about the dimensions of the eigenspaces. Define for each $k=1, \ldots, d$

$$
\begin{aligned}
\mu_{i}(k) & =\left\langle\frac{k \cdot a_{i}}{d}\right\rangle, \quad \mu(k)=\left(\mu_{i}(k)\right)_{i} \\
a_{i}(k) & =\mu_{i}(k) \cdot d \\
\sigma(k) & =\sum_{i=1}^{N} \mu_{i}(k)
\end{aligned}
$$

Here $\langle x\rangle=x-[x]$ denotes the fractional part of $x \in \mathbb{R}$. Further, let $s(k) \in$ $\{0, \ldots, N\}$ be the number of $a_{i}$ that are not equal to 0 modulo $d / \operatorname{gcd}(k, d)$. 
Lemma 6.3. With the notations above,

a) The rank of $\mathcal{L}_{k}^{1,0}$ is $\sigma(k)-1$, and the rank of $\mathcal{L}_{k}^{0,1}$ is $s(k)-1-\sigma(k)$.

b) $\mathbb{L}_{k}$ is a $\mathbb{C}$-local system of dimension $s(k)-2$, polarized by a hermitian form of signature $(\sigma(k)-1, s(k)-1-\sigma(k))$.

c) When $k$ is prime to $d$, an explicit basis of $\mathcal{L}_{k}^{1,0}$ is given by

$$
\omega_{k}^{l}=y^{-k} x^{l} f_{k} \mathrm{~d} x
$$

with $f_{k}=\prod_{j}\left(x-x_{j}\right)^{\left[\frac{k a_{j}}{d}\right]}$ where $l=0, \ldots, \sigma(k)-2$.

Proof. a) is shown in [Bou01, Lemma 4.5], and b) follows from a) and the fact that $\mathbb{L}_{k}$ is a polarized $\mathbb{C}$-VHS. Part c) is proved in [Bou05, Lemma 1.1.2]. In both source, our index $k$ is replaced by $d-k$.

The Galois conjugates of the local system $\mathbb{L}_{1}$ are parametrized by $k \in\{1, \ldots, d-$ $1\}$ such that $\operatorname{gcd}(k, d)=1$. We will speak of $\left(\mu_{i}(k)\right)_{i}$ as a Galois conjugate tuple. Furthermore, the local system $\mathbb{L}_{1}$ corresponds to a non-arithmetic lattice in $\mathrm{PU}(1, n)$ if and only if $\left(\mu_{i}\right)_{i}$ satisfies (INT) and at least one Galois conjugate $\mathbb{L}_{k}$ has signature different from $(0, n)$ and $(n, 0)$.

6.6. The period map. For each $k=1, \ldots, d-1$, where $\mathcal{L}_{k}^{1,0}$ is not trivial, we obtain a period map $\hat{B}_{0} \rightarrow \operatorname{Per}\left(\mathbb{L}_{k}\right)$ from the universal covering $\hat{B}_{0}$ of $B_{0}$ to the period domain of the VHS $\operatorname{Per}\left(\mathbb{L}_{k}\right)$. It is equivariant with respect to the action of $\pi_{1}\left(B_{0}\right)$ on $\hat{B}_{0}$ and on $\operatorname{Per}\left(\mathbb{L}_{k}\right)$ via the monodromy representation $\rho_{k}: \pi_{1}\left(B_{0}, \underline{x}^{\prime}\right) \rightarrow$ $\operatorname{Aut}\left(\left(\mathbb{L}_{k}\right)_{\underline{x}^{\prime}}\right)$. Let $K_{k}=\operatorname{ker}\left(\rho_{k}\right)$, and let $\widetilde{B}_{0}^{k}=\hat{B}_{0} / K_{k}$. Denote $p_{k}: \widetilde{B}_{0}^{k} \rightarrow \operatorname{Per}\left(\mathbb{L}_{k}\right)$ the resulting period map.

The map $p_{k}$ can be though of as a multi-valued map on $B_{0}$. For $\underline{x} \in B_{0}$ choose a path connecting $\underline{x}$ to $\underline{x}^{\prime}$. Via this path, the fibers of $R^{1} f_{*} \mathbb{C}$ above $\underline{x}$ and $\underline{x}^{\prime}$ can be canonically identified. Moreover,

$$
\left(R^{1} f_{*} \mathbb{C}\right)_{\underline{x^{\prime}}} \cong H^{1}\left(\mathcal{X}_{\underline{x}^{\prime}}, \mathbb{C}\right) \cong H_{\text {sing }}^{1}\left(\mathcal{X}_{\underline{x}^{\prime}}, \mathbb{C}\right)
$$

Hence, the inclusion

$$
H^{1,0}\left(\mathcal{X}_{\underline{x}}\right) \rightarrow H_{\text {sing }}^{1}\left(\mathcal{X}_{\underline{x}^{\prime}}, \mathbb{C}\right)
$$

is given by integration $\omega \mapsto\left(\gamma \mapsto \int_{\gamma} \omega\right)$. Since this inclusion is equivariant with respect to the $G$-action, it induces an inclusion

$$
\left(\mathcal{L}_{k}^{1,0}\right)_{\underline{x}} \cong H^{1,0}\left(\mathcal{X}_{\underline{x}}\right)_{\chi^{k}} \rightarrow H_{\text {sing }}^{1}\left(\mathcal{X}_{\underline{x}^{\prime}}, \mathbb{C}\right)_{\chi^{k}} \cong\left(\mathbb{L}_{k}\right)_{\underline{x}^{\prime}}
$$

and $p_{k}$ maps $\underline{x}$ to the point in $\operatorname{Per}\left(\mathbb{L}_{k}\right)$ defined by the image of this morphism. Since $p_{k}(\underline{x})$ depends on the chosen path, $p_{k}$ is multi-valued on $B_{0}$.

Assume for the rest of Section 6.6 that $\sum_{i} \mu_{i}(k)=2$ and $\mu_{i}(k) \notin \mathbb{Z}$ for all $i$. Then $\mathcal{L}_{k}^{1,0}$ is a line bundle with a global non-zero section $\omega_{k}(\underline{x})=\omega_{k}^{0}(\underline{x}) \in H^{0}\left(B_{0}, \mathcal{L}_{k}^{1,0}\right)$ and $\operatorname{Per}\left(\mathbb{L}_{k}\right)$ is a ball in a projective space of dimension $n=N-3$.

Lemma 6.4 ([DM86, Lemma 3.9]). Let $N \geq 4$. The (multi-valued) period map

$$
p_{k}: B_{0} \rightarrow \mathbb{P}\left(H^{1}\left(\mathcal{X}_{\underline{x}^{\prime}}, \mathbb{C}\right)_{\chi^{k}}\right), \quad \underline{x} \mapsto\left(\gamma \mapsto \int_{\gamma} \omega_{k}(\underline{x})\right)
$$

has injective differential for every $\underline{x} \in B_{0}$. It is given by the map

$$
\left(\mathcal{T} B_{0}\right)_{\underline{x}} \rightarrow \operatorname{Hom}\left(H^{1,0}\left(\mathcal{X}_{\underline{x}}\right)_{\chi^{k}}, \quad H^{1}\left(\mathcal{X}_{\underline{x}}, \mathbb{C}\right)_{\chi^{k}} / H^{1,0}\left(\mathcal{X}_{\underline{x}}\right)_{\chi^{k}}\right)
$$


mapping $\frac{\partial}{\partial v}$ to

$$
\left(\gamma \mapsto \frac{\partial}{\partial v} \int_{\gamma} \omega_{k}=\int_{\gamma} \frac{\partial}{\partial v} \omega_{k}\right)
$$

This implies that $p_{k}$ is a local isomorphism on $\widetilde{B}_{0}^{k}$, since the dimensions of the range and image agree. Recall also from Sec. 2.2.1 that the derivative of the period map is equal to the map $\widetilde{\tau}$, derived from the dual $\tau^{\vee}$ of the Higgs field.

6.6.1. Extending the period map. As in DM86, we consider the Fox completions $\widetilde{B}^{k}$ of $\widetilde{B}_{0}^{k}$ over $B^{k}=B^{\mu(k)}$ and $\widetilde{B}_{s s t}^{k}$ over $\bar{B}^{k}=\bar{B}^{\mu(k)}$. The period map $p_{k}$ has continuous extensions, denoted $p_{k}^{k}$, to $\widetilde{B}^{k}$ and to $\widetilde{B}_{s s t}^{k}$ (DM86, Sect. 8]), both of which are equivariant for the monodromy action of $\pi_{1}\left(B_{0}\right)$. The following diagram gives an overview of the spaces involved

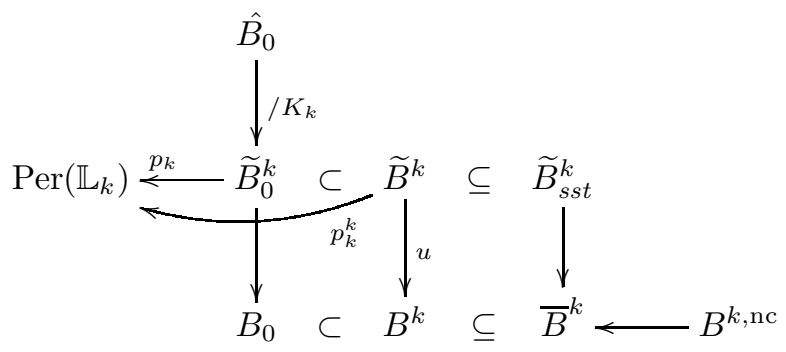

If there is additional symmetry in the tuple $\left(\mu_{i}(k)\right)_{i}$, then the above discussion carries over to a quotient (see Mos86]). Let $S \subset\{1, \ldots, N\}$, and assume $\mu_{i}(k)=$ $\mu_{j}(k)$ for all $i, j \in S$. The group $\Sigma=\operatorname{Sym}(S)$ acts on $B_{0}$, and there is an open, dense submanifold $F$ where the action is free. The VHS on $B_{0}$ descends to a VHS $\mathbb{L}_{k, \Sigma}$ on the quotient $F / \Sigma$, and we obtain a period map $\widehat{F / \Sigma} \rightarrow \operatorname{Per}\left(\mathbb{L}_{k, \Sigma}\right)$ from the universal covering of $F / \Sigma$ to the period domain of $\mathbb{L}_{k, \Sigma}$, which is equivariant for the monodromy action $\rho_{k, \Sigma}$ of $\pi_{1}(F / \Sigma)$. Set

$$
K_{k, \Sigma}=\operatorname{ker}\left(\rho_{k, \Sigma}\right) \quad \text { and } \quad \widetilde{F}_{\Sigma}^{k}=\widehat{F / \Sigma} / K_{k, \Sigma}
$$

and let

$$
p_{k, \Sigma}^{k}: \widetilde{B}_{\Sigma}^{k} \rightarrow \operatorname{Per}\left(\mathbb{L}_{k, \Sigma}\right)
$$

denote the induced map from the Fox completion of $\widetilde{F}_{\Sigma}^{k}$ over $B^{k} / \Sigma$ to the period domain of $\mathbb{L}_{k, \Sigma}$.

Remark. Assume that $\mu=\mu(1)$ satisfies (INT), then $p_{1}^{1}$ is an isomorphism from $\widetilde{B}^{1}$

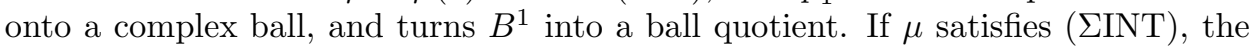
same is true for the induced map $p_{1, \Sigma}^{1}$ and $B^{1} / \Sigma$.

We shall also need the extension of the period map of $\mathbb{L}_{k}$ to the Fox completion

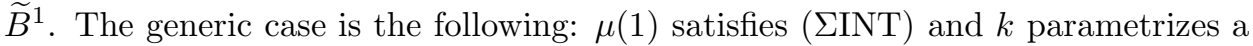
Galois conjugate $\mathbb{L}_{k, \Sigma}$ of $\mathbb{L}_{1, \Sigma}$. Since the kernels $K_{1, \Sigma}=K_{k, \Sigma}$, we obtain a period map

$$
p_{k, \Sigma}^{1}: \widetilde{F}_{\Sigma}^{1} \rightarrow \operatorname{Per}\left(\mathbb{L}_{k, \Sigma}\right)
$$

which we wish to extend to $\widetilde{B}_{\Sigma}^{1}$. Via $p_{1, \Sigma}^{1}$, the set $\widetilde{F}_{\Sigma}^{1}$ is the complement in $\mathbb{B}^{n}$ of normal crossing divisors, the preimages of the stable $\bar{L}_{i j}$ 's. By Lemma 6.6 below, 
the monodromy about each of these divisors is of finite order, whence by Gri70. Theorem 9.5] the map $p_{k, \Sigma}^{1}$ extends holomorphically to

$$
p_{k, \Sigma}^{1}: \widetilde{B}_{\Sigma}^{1} \rightarrow \operatorname{Per}\left(\mathbb{L}_{k, \Sigma}\right)
$$

with image in the interior of the period domain. The following diagram captures this situation.

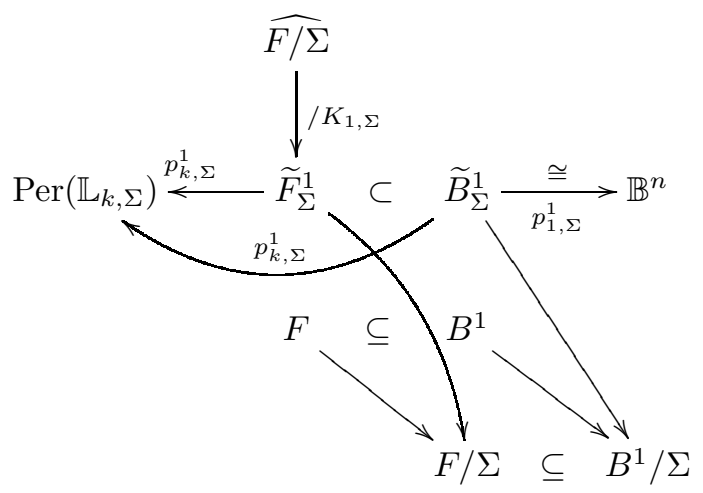

There is a third case, when we need an extension of $p_{k}$. As above let $k$ parameterize a Galois conjugate of $\mathbb{L}_{1}$, and let $\mathbb{L}_{1}$ have signature $(1, n)$, but do not assume that $\mu(1)$ satisfies (INT) (e.g. it only satisfies ([INT)). Let $\widetilde{B}_{1}^{1}$ denote the Fox completion of $\widetilde{B}_{0}^{1}$ over the union of $B_{0}$ with the codimension 1-strata of the $\mu(1)$ stable points. Then the map $p_{k}^{1}: \widetilde{B}_{0}^{1} \rightarrow \operatorname{Per}\left(\mathbb{L}_{k}\right)$ is again well-defined and extends to $p_{k}^{1}: \widetilde{B}_{1}^{1} \rightarrow \operatorname{Per}\left(\mathbb{L}_{k}\right)$. The point here is that $\widetilde{B}_{1}^{1}$ is a complex manifold by the discussion in [DM86, Sect. 10], the monodromy about the stable divisors being of finite order. We can thus apply the same reasoning as in the second case.

6.6.2. Relations between period maps. The period map, and in particular its domain of definition depends on $k$. If $p_{k}$ and $p_{\ell}$ belong to Galois conjugate local systems, their domains of definition coincide, but in general they need not be related in any way, as the following example shows.

Example 6.5. Consider the family of cyclic coverings $f: \mathcal{X} \rightarrow B_{0}$ of type $(12 ; 3,3,3,7,8)$. This family of curves is fiberwise a degree 2-covering of a family of cyclic coverings $f^{\prime}: \mathcal{X}^{\prime} \rightarrow B_{0}$ of type $(6 ; 3,3,3,1,2)$ by taking the quotient by $\left\langle g^{2}\right\rangle \subset G$, and the resulting map $\mathcal{X} \rightarrow \mathcal{X}^{\prime}$ induces an inclusion of VHS with image

$$
\bigoplus_{k \equiv 0 \bmod 2} \mathbb{L}_{k} \subset R^{1} f_{*} \mathbb{C}
$$

We neither have $K_{2} \subseteq K_{1}$, nor $K_{1} \subseteq K_{2}$. To see this, consider the monodromy transformation, i.e. the image of a small loop $\gamma_{i j}$ about one of the divisors $L_{i j}$ under the monodromy representation $\rho_{k}$. In case $\sum_{i} \mu_{i}(k)=2$ and $\mu_{i}(k) \notin \mathbb{Z}$, its order is the denominator of the reduced fraction $\left|1-\mu_{i}(k)-\mu_{j}(k)\right|^{-1}$ or $\infty$ if $\mu_{i}(k)+\mu_{j}(k)=1$. Thus, $L_{12}$ is a parabolic divisor for $\mathbb{L}_{2}$, which is elliptic for $\mathbb{L}_{1}$, whereas e.g. for $L_{15}$, the monodromy transformation has order 12 for $k=1$ and order 3 for $k=2$.

In particular, the pullback of the local system $\mathbb{L}_{2}$ to the universal cover $\widetilde{B}^{1} \cong \mathbb{B}^{n}$ of $B^{1}$ cannot be extended as local system over the preimage of $L_{12}$ in $\mathbb{B}^{n}$, thus for a generic geodesic, parallel transport is not defined for all times. 
For each of the non-arithmetic ball quotients listed in the table in Section 6.3 there exists a sub-VHS for which an analogous statement holds.

For later use, we record a relation between $\left(\mu_{i}(1)\right)_{i}$ and $\left(\mu_{i}(k)\right)_{i}$ for $k$ prime to $d$. Its proof is a straightforward computation.

Lemma 6.6. Let $d>1, k \in\{0, \ldots, d-1\}$ be prime to $d$, and let $i, j \in\{1, \ldots, N\}$. Then

a) $\mu_{i}(k)+\mu_{j}(k)=1$ iff $\mu_{i}(1)+\mu_{j}(1)=1$

b) If $\kappa_{i j}=\left(1-\mu_{i}(1)-\mu_{j}(1)\right)^{-1}$ is in $\mathbb{Z}$ or if $a_{i}=a_{j}, \kappa_{i j} \in \frac{1}{2} \mathbb{Z}$ and $k$ is odd, then

$$
\kappa_{i j} \cdot\left(1-\mu_{i}(k)-\mu_{j}(k)\right) \in \mathbb{Z} \text {. }
$$

6.6.3. Explicit description of the period map. We describe a trivialization of $\mathbb{L}_{k}$ at $\underline{x} \in B_{0}$. Instead of working with $\left(\mathbb{L}_{k}\right)_{\underline{x}}=H^{1}\left(\mathcal{X}_{\underline{x}}, \mathbb{C}\right)_{\chi^{k}}$, we use the isomorphism

$$
H^{1}\left(\mathcal{X}_{\underline{x}}, \mathbb{C}\right)_{\chi^{k}} \cong H^{1}\left(\mathbb{P}^{1} \backslash\{\underline{x}\}, L\right)
$$

where $\{\underline{x}\}$ is the set of points defined by $\underline{x}$ and $L$ is the rank one local system on $\mathbb{P}^{1} \backslash\{\underline{x}\}$ having local monodromy $\exp \left(2 \pi i \mu_{i}(k)\right)$ about $x_{i}$ (see [DM86, Sect. 2] for a discuss of the cohomology of $L)$. Explicitly, $H^{*}\left(\mathbb{P}^{1} \backslash\{\underline{x}\}, L\right)$ can be computed using the de Rham complex of $\mathrm{C}^{\infty}$-forms on $\mathbb{P}^{1} \backslash\{\underline{x}\}$. One can also consider the subcomplex of compactly supported forms, whose cohomology we denote by $H_{c}^{*}\left(\mathbb{P}^{1} \backslash\{\underline{x}\}, L\right)$.

If none of the $\mu_{i}(k)$ is an integer, then $H_{c}^{*}\left(\mathbb{P}^{1} \backslash\{\underline{x}\}, L\right) \cong H^{*}\left(\mathbb{P}^{1} \backslash\{\underline{x}\}, L\right)$ (see [DM86, 2.3]). The Poincaré duality pairing

$$
H^{i}\left(\mathbb{P}^{1} \backslash\{\underline{x}\}, L\right) \otimes H_{c}^{2-i}\left(\mathbb{P}^{1} \backslash\{\underline{x}\}, L^{\vee}\right) \rightarrow \mathbb{C}, \quad \alpha \otimes \beta \mapsto \int_{\mathbb{P}^{1} \backslash\{\underline{x}\}} \alpha \wedge \beta
$$

allows us to identify the dual of $H^{1}\left(\mathbb{P}^{1} \backslash\{\underline{x}\}, L\right)$ with the first cohomology of the complex of currents on $\mathbb{P}^{1} \backslash\{\underline{x}\}$ with values in $L^{\vee}$. We describe a particular basis of $H^{1}\left(\mathbb{P}^{1} \backslash\{\underline{x}\}, L^{\vee}\right)$ (compare [DM86, 2.5]). Assume $\{1, \ldots, N\}=S_{1} \cup S_{2}$ is a partition such that $\sum_{i \in S_{1}} \mu_{i}(k) \notin \mathbb{Z}$. Embed the union of two trees $T_{1} \cup T_{2}$ into $\mathbb{P}^{1}$ such that the vertices of $T_{i}$ are $\left\{x_{s} \mid s \in S_{i}\right\}$, and let $\gamma_{j}$ be the oriented edges of $T_{1} \cup T_{2}$. Then a basis of $H^{1}\left(\mathbb{P}^{1} \backslash\{\underline{x}\}, L^{\vee}\right)$ is given by the currents of integration along the paths $\gamma_{j}, j=1, \ldots, N-2$, each tensored with a global section $e_{j}$ of $L^{\vee}$ restricted to (the interior of) $\gamma_{j}$.

If $\mathcal{L}_{k}^{1,0}$ is a line bundle, the basis element of Lemma 6.3 is identified by the isomorphism (21) with the multi-valued form $\omega_{k}=\prod_{j}\left(x-x_{j}\right)^{-\mu_{j}(k)} \mathrm{d} x$ tensored with an appropriate multi-valued section $e$ of $L$, so as to produce an honest singlevalued section of $H^{0}\left(\mathbb{P}^{1} \backslash\{\underline{x}\}, \Omega^{1}(L)\right) \subset H^{1}\left(\mathbb{P}^{1} \backslash\{\underline{x}\}, L\right)$. The period map is then the map

$$
p_{k}=\left(F_{1}^{k}: \cdots: F_{N-2}^{k}\right) \text { with } F_{j}^{k}=\int_{\gamma_{j} \otimes e_{j}} \omega_{k} \otimes e .
$$

\section{LyapunOV EXPONENTS OF BALL QUOTIENTS CONSTRUCTED VIA CYCLIC COVERINGS}

In this section we calculate explicitly the Lyapunov exponents for all non-arithmetic ball quotients arising from cyclic covers. In the table below we also add the set of 
relative orbifold Euler numbers for an easy comparison. Their defintion is given in the next section.

Theorem 7.1. The non-negative distinct Lyapunov exponents of the primitive part of the two-dimensional non-arithmetic ball quotients arising from cyclic coverings are given in the following table. Here, $\mathbb{U}$ denotes the maximal unitary subsystem of $\mathbb{P}$.

\begin{tabular}{|c|c|c|c|c|c|c|}
\hline & $d$ & $g$ & $\operatorname{dim}_{\mathbb{R}} \mathbb{P}$ & $\operatorname{dim}_{\mathbb{R}} \mathbb{U}$ & Prim. Lyapunov spectrum & Relative $e^{\text {orb }}$ \\
\hline 1 & $\overline{12}$ & $\overline{12}$ & 12 & 0 & $\{1, \quad 5 / 13, \quad 0\}$ & $\{1, \quad 1 / 13\}$ \\
\hline 2 & $\overline{12}$ & $\overline{12}$ & $\overline{12}$ & 0 & $\{1, \quad 5 / 17, \quad 0\}$ & $\begin{array}{l}1 / 17\} \\
\end{array}$ \\
\hline 3 & 12 & 12 & 12 & 0 & $\begin{array}{lll}\{1, & 7 / 22, & 0\end{array}$ & $1 / 22\}$ \\
\hline 4 & $\overline{12}$ & 11 & 12 & 0 & $\{1, \quad 5 / 13, \quad 0\}$ & $\{1, \quad 1 / 13\}$ \\
\hline 5 & 15 & 18 & 24 & 6 & $\{1, \quad 16 / 37, \quad 7 / 37, \quad 0\}$ & $\{1, \quad 4 / 37, \quad 1 / 37\}$ \\
\hline 6 & 18 & 25 & 18 & 6 & $\{1, \quad 5 / 16, \quad 0\}$ & $\{1, \quad 1 / 16\}$ \\
\hline 7 & 18 & 25 & 18 & 6 & $5 / 16, \quad 0\}$ & $\{1, \quad 1 / 16\}$ \\
\hline 8 & 20 & 22 & $\overline{24}$ & 6 & $\{1, \quad 4 / 11, \quad 3 / 11$ & $\{1, \quad 4 / 33, \quad 1 / 33\}$ \\
\hline 9 & 20 & 23 & $\overline{24}$ & 6 & $\{1, \quad 4 / 11, \quad 3 / 11$, & $\{1, \quad 4 / 33$, \\
\hline$\overline{10}$ & 20 & 27 & 24 & $\overline{12}$ & $\{1, \quad 11 / 46, \quad 0\}$ & $\{1,1 / 46\}$ \\
\hline 11 & 24 & 30 & 24 & 12 & $\{1, \quad 7 / 22, \quad 0\}$ & $1 / 22\}$ \\
\hline 12 & 24 & 31 & 24 & 12 & $\{1, \quad 7 / 22, \quad 0\}$ & $\{1, \quad 1 / 22\}$ \\
\hline 13 & 30 & 37 & 24 & 6 & $\{1, \quad 16 / 37, \quad 7 / 37$, & $\{1, \quad 4 / 37, \quad 1 / 37\}$ \\
\hline$\overline{14}$ & 42 & 52 & 36 & 18 & $\{1, \quad 16 / 61, \quad 13 / 61, \quad 0\}$ & $\{1, \quad 4 / 61, \quad 1 / 61\}$ \\
\hline 15 & 42 & 58 & 36 & 18 & $16 / 61, \quad 13 / 61$ & $\{1, \quad 4 / 61, \quad 1 / 61\}$ \\
\hline
\end{tabular}

For the three-dimensional ball quotient, the positive primitive Lyapunov spectrum is given by

$$
1,1, \frac{25}{93}, \frac{25}{93}, 0,0,0,0 \text {. }
$$

Corollary 7.2. The non-arithmetic lattices in $\mathrm{PU}(1, n)$ arising via cyclic coverings fall into precisely nine commensurability classes.

Proof. Using the trace field, we only need to decide whether no. 2 or 3 belong to the class of $\{1,4\}$ or are commensurable and whether no. 10 belongs to the class of $\{8,9\}$. But by Corollary 4.5 the primitive Lyapunov spectrum is a commensurability invariant among lattices admitting a modular embedding.

The proof of the theorem relies on computing the right-hand side of (2). We first show how to relate this quantity to contributions coming from the boundary divisors, and then compute these contributions for each case.

7.1. Intersection products of Chern classes. Let $B^{u}=\mathbb{B}^{n} / \Gamma^{\prime}$ be a ball quotient, where $\Gamma^{\prime} \subset \mathrm{PU}(1, n)$ acts cofinitely and freely on $\mathbb{B}^{n}$. We assume that there is a smooth projective variety $Y$ such that $B^{u}$ embeds into $Y$ with $Y \backslash B^{u}=\Delta$, a divisor with normal crossings. Suppose further that we are given a polarized $\mathbb{C}$-VHS $\mathbb{L}^{u}$ on $B^{u}$ of weight 1 and signature $(1, n)$, whose local monodromies are unipotent. Recall from Section 2.2.1 that Higgs field $\tau$ of $\mathbb{L}^{u}$ comes with two derived maps $\widetilde{\tau}$ and $\tau^{\vee}$.

Lemma 7.3. Assume that $\widetilde{\tau}$ (or equivalently, $\tau^{\vee}$ ) is injective. Then

$$
\frac{(n+1) \mathrm{c}_{1}\left(\mathcal{E}^{1,0}\right) \cdot \mathrm{c}_{1}\left(\omega_{Y}\right)^{n-1}}{\mathrm{c}_{1}\left(\omega_{Y}\right)^{n}}=1-\frac{\mathrm{c}_{1}\left(\operatorname{coker} \tau^{\vee}\right) \cdot \mathrm{c}_{1}\left(\omega_{Y}\right)^{n-1}}{\mathrm{c}_{1}\left(\omega_{Y}\right)^{n}}
$$


where $\omega_{Y}=\bigwedge^{n} \Omega_{Y}(\log \Delta)$.

Proof. From the assumption, we obtain a short exact sequence

$$
0 \rightarrow \mathcal{E}^{1,0} \otimes \mathcal{T}_{Y}(-\log \Delta) \rightarrow \mathcal{E}^{0,1} \rightarrow \operatorname{coker} \tau^{\vee} \rightarrow 0 .
$$

By [EV02, Theorem 1.1], we have

$$
\mathrm{c}_{1}\left(\mathcal{E}^{1,0} \oplus \mathcal{E}^{0,1}\right)=0
$$

and from the above exact sequence we obtain

$$
\mathrm{c}_{1}\left(\mathcal{E}^{0,1}\right)=\mathrm{c}_{1}\left(\mathcal{T}_{Y}(-\log \Delta)\right)+n \cdot \mathrm{c}_{1}\left(\mathcal{E}^{1,0}\right)+\mathrm{c}_{1}\left(\operatorname{coker} \tau^{\vee}\right) .
$$

Combining these two equations yields

$$
(n+1) \mathrm{c}_{1}\left(\mathcal{E}^{1,0}\right) \cdot \mathrm{c}_{1}\left(\omega_{Y}\right)^{n-1}=-\left(\mathrm{c}_{1}\left(\mathcal{T}_{Y}(-\log \Delta)\right)+\mathrm{c}_{1}\left(\operatorname{coker} \tau^{\vee}\right)\right) \cdot \mathrm{c}_{1}\left(\omega_{Y}\right)^{n-1} .
$$

Remark 7.4. For the computation of $\mathrm{c}_{1}\left(\operatorname{coker} \tau^{\vee}\right)$, it will be convenient to consider the following short exact sequence associated with $\widetilde{\tau}$

$$
0 \rightarrow \mathcal{T}_{Y}(-\log \Delta) \rightarrow \mathcal{H o m}\left(\mathcal{E}^{1,0}, \mathcal{E}^{0,1}\right) \rightarrow \operatorname{coker} \widetilde{\tau} \rightarrow 0 .
$$

Then $\mathrm{c}_{1}(\operatorname{coker} \widetilde{\tau})=\mathrm{c}_{1}\left(\operatorname{coker} \tau^{\vee}\right)$, using additivity of $\mathrm{c}_{1}$ on short exact sequences.

7.2. Computation of $c_{1}\left(\operatorname{coker} \tau^{\vee}\right)$ for the case (INT). Let $N \geq 4$, and let $f: \mathcal{X} \rightarrow B_{0}$ be a cyclic covering of type $\left(d, a_{1}, \ldots, a_{N}\right)$, such that the collection $\mu_{i}=a_{i} / d$ satisfies (INT). Let $B \cong \mathbb{B}^{n} / \Gamma$ be the ball quotient parametrizing $\mu$-stable points. Let $1 \leq k \leq d-1$ be coprime to $d$, and such that the direct summand $\mathbb{L}=\mathbb{L}_{k}$ in the VHS of $f$ is polarized by a hermitian form of signature $(1, n)$.

We consider a finite index normal subgroup $\Gamma^{\prime}$ of $\Gamma$ such that the local monodromies about the boundary divisors are unipotent under $\rho_{k}$, and such that moreover $\Gamma^{\prime}$ acts freely on $\mathbb{B}^{n}$. We denote $\pi: B^{u} \rightarrow B$ and $u: \mathbb{B}^{n} \rightarrow \mathbb{B}^{n} / \Gamma^{\prime}$ the two projections, and let $\mathbb{L}^{u}$ be the pullback of $\mathbb{L}$ to $\pi^{-1}\left(B_{0}\right) \subset B^{u}$. By the discussion in Section 6.6.1, we can extend $\mathbb{L}^{u}$ to a VHS on $B^{u}$, and we denote it by the same letter.

The results of the preceding sections apply to $\mathbb{L}^{u}$. Let $\tau^{\vee}=\tau_{k}^{\vee}$ be the dual of the Higgs field, and let $p_{k}=p_{k}^{1}$ be the period map for $\mathbb{L}$, which we can also think of as a multi-valued map on $B^{u}$. We have

\section{Lemma 7.5.}

$$
\mathrm{c}_{1}\left(\operatorname{coker} \tau^{\vee}\right)=\sum_{\substack{\{i, j\}: \\ \mu_{i}+\mu_{j}<1}} n_{i j}^{k} \cdot \sum_{\substack{L \subset \pi^{-1} L_{i j} \\ \text { irred. }}}[L]+\sum_{\substack{\Delta^{\prime} \subset \Delta \\ \text { irred. }}} n_{\Delta^{\prime}} \Delta^{\prime}
$$

Moreover, $n_{i j}^{k}$ is the vanishing order of $\operatorname{det}\left(J\left(p_{k}\right)\right)$ at a point of $\pi^{*} L_{i j}^{\circ}$.

Proof. Define the line bundles

$$
\mathcal{L}_{1}=\bigwedge^{n} \mathcal{T}_{B}(-\log \Delta) \quad \text { and } \quad \mathcal{L}_{2}=\bigwedge^{n} \mathcal{H o m}\left(\mathcal{E}^{1,0}, \mathcal{E}^{0,1}\right)
$$

Then

$$
\mathrm{c}_{1}\left(\operatorname{coker} \tau^{\vee}\right)=\mathrm{c}_{1}\left(\mathcal{L}_{2}\right)-\mathrm{c}_{1}\left(\mathcal{L}_{1}\right) .
$$

Let $\phi: \mathcal{L}_{1} \rightarrow \mathcal{L}_{2}$ be the map induced by $\widetilde{\tau}_{k}$. It is locally at $p \in Y$ given by multiplication with $a_{p} \in \mathcal{O}_{Y, p}$. We tensor both line bundles with $\mathcal{L}_{2}^{-1}$. Then the 
image of $\mathcal{L}_{1} \otimes \mathcal{L}_{2}^{-1}$ under $\phi \otimes$ id is the ideal sheaf $\mathcal{I} \subseteq \mathcal{O}_{Y}$ locally generated by $a_{p}$. Moreover,

$$
\mathrm{c}_{1}(\mathcal{I})=-\mathrm{c}_{1}(\operatorname{coker} \tilde{\tau}),
$$

and $\mathrm{c}_{1}(\mathcal{I})=-D$ for some divisor $D$ on $Y$.

It follows from Lemma 6.4 that $a_{p}=1$ if $p \in \pi^{-1}\left(B_{0}\right)$. Hence,

$$
\operatorname{supp}(-D) \subseteq \bigcup_{(i, j): \mu_{i}+\mu_{j}<1} \pi^{-1} L_{i j} \cup \Delta .
$$

Let $p \in \pi^{-1} L_{i j}^{\circ}$, and let $L$ be the irreducible component of $\pi^{*} L_{i j}$ containing $p$. Then $\operatorname{ord}_{L}(-D)=\operatorname{ord}_{L}\left(a_{p}\right)$. Since $\phi=\wedge^{n} \widetilde{\tau}$ and since in the interior, $\widetilde{\tau}$ is the derivative of the period map $p_{k}$, it follows that $\operatorname{ord}_{L}\left(a_{p}\right)$ is independent of the irreducible component of $\pi^{-1} L_{i j}$, since $p_{k}$ is equivariant under the full group $\Gamma$, and given by the vanishing order of $\operatorname{det}\left(J\left(p_{k}\right)\right)$ along a component of the preimage of $L_{i j}$.

7.2.1. Near elliptic divisors. Next we study $\widetilde{\tau}_{k}$ at one of the elliptic divisors $L_{i j}$. By Lemma 6.6, $\mu_{i}(k)+\mu_{j}(k) \neq 1$, and

$$
\ell_{i j}^{k}=\kappa_{i j} \cdot\left|1-\mu_{i}(k)-\mu_{j}(k)\right| \in \mathbb{N} .
$$

Lemma 7.6. We have

$$
n_{i j}^{k}= \begin{cases}\ell_{i j}^{k}-1 & \text { if } \mu_{i}(k)+\mu_{j}(k)<1 \\ n \cdot \ell_{i j}^{k}-1 & \text { if } \mu_{i}(k)+\mu_{j}(k)>1\end{cases}
$$

To prove this lemma, we will need an explicit description of the period map locally about $L_{i j}$. We recall the discussion in DM86. Consider a neighborhood of a point

$$
\underline{x}^{\prime}=\left(x_{1}^{\prime}, \ldots, x_{N}^{\prime}\right) \in \mathrm{PGL}_{2}(\mathbb{C}) \backslash\left(\mathbb{P}^{1}\right)^{N},
$$

where precisely two entries coincide. Up to renumbering, we can assume them to be the first two. Lift the point to $\mathbb{C}^{N}$ by normalizing $x_{1}^{\prime}=x_{2}^{\prime}=0, x_{N-1}^{\prime}=a, x_{N}^{\prime}=b$ with $a, b \in \mathbb{C}$ some suitable points. Let $\varepsilon$ be so small that the disks of radius $\varepsilon$ about the $x_{i}^{\prime}$ do not intersect, and let $U$ be the set of

$$
\underline{x}=\left(x_{1}=0, x_{2}, \ldots, x_{N-2}, x_{N-1}=a, x_{N}=b\right)
$$

such that $\left|x_{i}-x_{i}^{\prime}\right|<\varepsilon$. Then $U$ describes a neighborhood of $\underline{x}^{\prime} \in B$.

About $\underline{x} \in U$, we can choose a basis of $H^{1}\left(\mathbb{P}^{1} \backslash\{\underline{x}\}, L\right)$ as follows (compare Section 6.6.3). Let $\gamma_{1}$ be the straight line segment connecting $x_{1}=0$ and $x_{2}$. Embed a tree $T$ into $\mathbb{C} \backslash B_{\varepsilon}(0)$ such that its vertices are $x_{i}, i=3, \ldots, N$, and let $\gamma_{j}(j=2, \ldots, N-2)$ be the edges of $T$ (with a chosen orientation). For each $j=1, \ldots, N-2$, choose a section $e_{j}$ of $L^{\vee}$ on interior of $\gamma_{j}$.

With this choice of basis, we obtain in particular for the first of the components $F_{j}^{k}$ of $p_{k}$

$$
\begin{aligned}
F_{1}^{k}(\underline{x}) & =\int_{0}^{x_{2}} x^{-\mu_{1}(k)}\left(x-x_{2}\right)^{-\mu_{2}(k)} \prod_{i \neq 1,2}\left(x-x_{i}\right)^{-\mu_{i}(k)} \mathrm{d} x \\
& =x_{2}^{1-\mu_{1}(k)-\mu_{2}(k)} \int_{0}^{1} x^{-\mu_{1}(k)}(x-1)^{-\mu_{2}(k)} \prod_{i \neq 1,2}\left(x_{2} x-x_{i}\right)^{-\mu_{i}(k)} \mathrm{d} x
\end{aligned}
$$


The factor

$$
I_{k}(\underline{x})=\int_{0}^{1} x^{-\mu_{1}(k)}(x-1)^{-\mu_{2}(k)} \prod_{i \neq 1,2}\left(x_{2} x-x_{i}\right)^{-\mu_{i}(k)} \mathrm{d} x,
$$

and $I_{k}$ is well-defined at $\left\{x_{2}=0\right\}$. Moreover (compare DM86, 9.5]), $I_{k}$ is holomorphic and $I_{k} \neq 0$ at $\left\{x_{2}=0\right\}$, so we may suppose that $I_{k}$ does not vanish on $U$.

The case $\mu_{i}(k)+\mu_{j}(k)<1$. The above discussion, together with Lemma 6.4 yields the following lemma, see DM86, 9.5].

Lemma 7.7. a) If $\mu_{i}(k)+\mu_{j}(k)<1$, then in a neighborhood $U$ of the divisor $L_{i j}$, the period map is a multi-valued map to $\mathbb{P}^{n}$, locally given as

$$
\left(z_{1}, \ldots, z_{n}\right) \mapsto\left(z_{1}^{1-\mu_{i}(k)-\mu_{j}(k)}, z_{2}, \ldots, z_{n}\right),
$$

where $z_{1}=0$ is a local equation for $L_{i j}$.

b) Assume that $\left(\mu_{i}(1)\right)_{i}$ satisfies (INT). The ramification order of $\pi: B^{u} \rightarrow B$ at $L_{i j}$ is

$$
\kappa_{i j}=\left(1-\mu_{i}-\mu_{j}\right)^{-1}
$$

Proof of Lemma 7.6. first case. The period map $p_{k}$ thought of as a multi-valued map on $B^{u}$ is locally about $L_{i j}$ given as the composition

$$
\left(\left(z_{1}, \ldots, z_{n}\right) \mapsto\left(z_{1}^{1-\mu_{i}(k)-\mu_{j}(k)}, z_{2}, \ldots, z_{n}\right)\right) \circ\left(\left(z_{1}, \ldots, z_{n}\right) \mapsto\left(z_{1}^{\kappa_{i j}}, z_{2}, \ldots, z_{n}\right)\right) .
$$

Hence, $\operatorname{det}\left(J\left(p_{k}\right)\right)=\ell_{i j}^{k} z_{1}^{\ell_{i j}^{k}-1}$.

The case $\mu_{i}(k)+\mu_{j}(k)>1$. Then the multivalued function

$$
F_{1}^{k}=x_{2}^{1-\mu_{1}(k)-\mu_{2}(k)} I_{k}
$$

has a pole at $x_{2}=0$ of order $\left|1-\mu_{1}(k)-\mu_{2}(k)\right| \in \mathbb{Q}$ and the projective tuple

$$
\left(x_{2}^{\mu_{1}(k)+\mu_{2}(k)-1} F_{1}^{k}: \cdots: x_{2}^{\mu_{1}(k)+\mu_{2}(k)-1} F_{N-2}^{k}\right)
$$

is well-defined on $U$. We set $m=\mu_{1}(k)+\mu_{2}(k)-1$ and dehomogenize $p_{k}$ with respect to the first coordinate

$$
\begin{aligned}
p_{k}(\underline{x}) & =\left(F_{2}^{k} / F_{1}^{k}, \ldots, F_{N-2}^{k} / F_{1}^{k}\right) \\
& =\left(x_{2}^{m} F_{2}^{k} / I_{k}, \ldots, x_{2}^{m} F_{N-2}^{k} / I_{k}\right) .
\end{aligned}
$$

For the computations below the following lemma is useful. Its proof is a straightforward computation.

Lemma 7.8. Let $U \subset \mathbb{C}^{n}$,

$$
f: U \rightarrow \mathbb{P}^{n}, \quad w=\left(w_{1}, \ldots, w_{n}\right) \mapsto\left(f_{0}(w): f_{1}(w): \cdots: f_{n}(w)\right)
$$

be a holomorphic map, and let $w \in U$ be such that $f_{0}(w) \neq 0$. Then in an open neighborhood of $w$ the determinants of

$$
J_{1}=\left(\frac{\partial\left(f_{i} / f_{0}\right)}{\partial w_{j}}\right)_{i, j} \quad \text { and } \quad J_{2}=\left(\begin{array}{cccc}
f_{0} & \frac{\partial f_{0}}{\partial w_{1}} & \cdots & \frac{\partial f_{0}}{\partial w_{n}} \\
f_{1} & \frac{\partial f_{1}}{\partial w_{1}} & \cdots & \frac{\partial f_{1}}{\partial w_{n}} \\
\vdots & \vdots & \ddots & \vdots \\
f_{n} & \frac{\partial f_{n}}{\partial w_{1}} & \cdots & \frac{\partial f_{n}}{\partial w_{n}}
\end{array}\right)
$$


are related by

$$
\operatorname{det}\left(J_{2}\right)=f_{0}^{n+1} \operatorname{det}\left(J_{1}\right)
$$

We would like to determine the vanishing order of the determinant of period $\operatorname{map} \bar{p}_{k}$ at a point $\underline{x}=\left(x_{1}=0, x_{2}=0, x_{3}, \ldots, x_{N}\right)$. Note that

$$
\left(\frac{\partial\left(f_{i} / f_{0}\right)}{\partial x_{j}}\right)_{i, j}=f_{0}^{-2}\left(\left|\begin{array}{cc}
f_{0} & f_{i} \\
\frac{\partial f_{0}}{\partial x_{j}} & \frac{\partial f_{i}}{\partial x_{j}}
\end{array}\right|\right)_{i, j}
$$

and recall that only $x_{2}, \ldots, x_{n+1}$ are varying coordinates. We have to evaluate the vanishing order of the determinant of the following matrix. We suppress the superand subscript $k$ in the following.

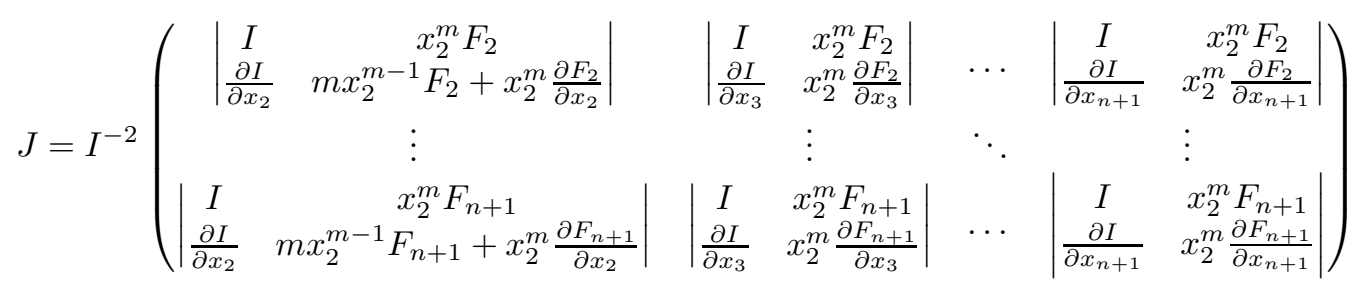

Lemma 7.9. J vanishes of order $x_{2}^{n m-1}$ at $\underline{x}$.

Proof. Write $\operatorname{det}(J)=I^{-2} x_{2}^{(n-1) m+m-1} \operatorname{det}(\widetilde{J})$ with

$$
\widetilde{J}=\left(\begin{array}{cc|c|c|cc}
\left|\begin{array}{cc}
I & x_{2} F_{2} \\
\frac{\partial I}{\partial x_{2}} & m F_{2}+x_{2} \frac{\partial F_{2}}{\partial x_{2}}
\end{array}\right| & \left|\begin{array}{ccc}
I & F_{2} \\
\frac{\partial I}{\partial x_{3}} & \frac{\partial F_{2}}{\partial x_{3}}
\end{array}\right| & \ldots & \left|\begin{array}{cc}
I & F_{2} \\
\frac{\partial I}{\partial x_{n+1}} & \frac{\partial F_{2}}{\partial x_{n+1}}
\end{array}\right| \\
\vdots & \vdots & \ddots & \vdots \\
\left|\begin{array}{cc}
I & x_{2} F_{n+1} \\
\frac{\partial I}{\partial x_{2}} & m F_{n+1}+x_{2} \frac{\partial F_{n+1}}{\partial x_{2}}
\end{array}\right| & \left|\begin{array}{cc|cc}
I & F_{n+1} \\
\frac{\partial I}{\partial x_{3}} & \frac{\partial F_{n+1}}{\partial x_{3}}
\end{array}\right| & \ldots & \left|\begin{array}{cc}
I & F_{n+1} \\
\frac{\partial I}{\partial x_{n+1}} & \frac{\partial F_{n+1}}{\partial x_{n+1}}
\end{array}\right|
\end{array}\right)
$$

We show that $\widetilde{J}$ does not vanish at $\underline{x}=\left(0, x_{3}, \ldots, x_{n+1}\right)$. Evaluation at $\underline{x}$ yields

$$
\begin{aligned}
& \operatorname{det}(\widetilde{J})=m I\left|\begin{array}{cccc}
F_{2} & I \frac{\partial F_{2}}{\partial x_{3}}-F_{2} \frac{\partial I}{\partial x_{3}} & \ldots & I \frac{\partial F_{2}}{\partial x_{n+1}}-F_{2} \frac{\partial I}{\partial x_{n+1}} \\
\vdots & \vdots & \ddots & \vdots \\
F_{n+1} & I \frac{\partial F_{n+1}}{\partial x_{3}}-F_{n+1} \frac{\partial I}{\partial x_{3}} & \ldots & I \frac{\partial F_{n+1}}{\partial x_{n+1}}-F_{n+1} \frac{\partial I}{\partial x_{n+1}}
\end{array}\right| \\
& =m I\left|\begin{array}{cccc}
F_{2} & I \frac{\partial F_{2}}{\partial x_{3}} & \ldots & I \frac{\partial F_{2}}{\partial x_{n+1}} \\
\vdots & \vdots & \ddots & \vdots \\
F_{n+1} & I \frac{\partial F_{n+1}}{\partial x_{3}} & \cdots & I \frac{\partial F_{n+1}}{\partial x_{n+1}}
\end{array}\right| \\
& =m I^{1+(n-1)}\left|\begin{array}{cccc}
F_{2} & \frac{\partial F_{2}}{\partial x_{3}} & \cdots & \frac{\partial F_{2}}{\partial x_{n+1}} \\
\vdots & \vdots & \ddots & \vdots \\
F_{n+1} & \frac{\partial F_{n+1}}{\partial x_{3}} & \cdots & \frac{\partial F_{n+1}}{\partial x_{n+1}}
\end{array}\right|
\end{aligned}
$$

By Lemma 6.4 and Lemma 7.8, we know that the determinant of

$$
\left(\begin{array}{cccc}
F_{2} & \frac{\partial F_{2}}{\partial x_{3}} & \cdots & \frac{\partial F_{2}}{\partial x_{n+1}} \\
\vdots & \vdots & \ddots & \vdots \\
F_{n+1} & \frac{\partial F_{n+1}}{\partial x_{3}} & \cdots & \frac{\partial F_{n+1}}{\partial x_{n+1}}
\end{array}\right)
$$


does not vanish at a point $\left(x_{3}, \ldots, x_{n+1}\right)$ where all coordinates are distinct and different from $0, x_{N-1}, x_{N}$, since this matrix describes the period map of the tuple

$$
\mu^{\prime}=\left(\mu_{1}(k)+\mu_{2}(k), \mu_{2}(k), \ldots, \mu_{N}(k)\right) .
$$

When $N=4$, the above determinant degenerates to $F_{2}$, whose non-vanishing is the content of [DM86, Proposition 2.13].

Proof of Lemma 7.6, second case. If we precompose the period map starting on $U$ with $\pi: B^{u} \rightarrow B$, then using the chain rule, we see that $\operatorname{det}\left(J\left(p_{k}\right)\right)$ vanishes of order $\kappa_{i j} n m-1$ at a generic point of the preimage of $L_{i j}$.

\subsection{Near parabolic divisors.}

Lemma 7.10. We have $n_{\Delta^{\prime}}=0$ for every irreducible component $\Delta^{\prime}$ of $\Delta$.

Proof. By Lemma 6.4 the period map is a local isomorphism away from the elliptic divisors, hence in particular near a generic point of a parabolic boundary divisor. It thus suffices to prove the lemma for a uniformizing VHS.

To emphasize similarity with the case of the universal family of elliptic curves, we use the Siegel domain realization

$$
\mathbb{B}_{\text {Siegel }}=\left\{\left(z_{1}: z_{2}: \ldots: z_{n}: 1\right): \operatorname{Im}\left(z_{1}\right)-\sum_{i=2}^{n}\left|z_{i}\right|^{2}>0\right\} \subset \mathbb{P}^{n}
$$

and work near the boundary point $(1: 0: \cdots: 0) \in \mathbb{P}^{n}$. A loop around the boundary point is represented by the parabolic matrix $T=I_{n+1}+E_{1, n+1}$. We write $q=\exp \left(2 \pi i z_{1}\right)$.

We also work with the dual uniformizing VHS, where now $\mathbb{W}^{1,0}$ has rank $n$. Over $\mathbb{B}_{\text {Siegel }}$ we choose a basis $s_{1}, \ldots, s_{n+1}$ of the constant local system and define the Hodge filtration $\mathcal{W}^{1,0}$ as the kernel of the tautological quotient map

$$
\mathbb{C}^{n+1} \otimes \mathcal{O}_{\mathbb{B}_{\text {Siegel }}} \rightarrow \mathcal{O}_{\mathbb{B}_{\text {Siegel }}}, \quad \sum_{i=1}^{n+1} f_{i} s_{i} \mapsto\left(\sum_{i=1}^{n} z_{i} f_{i}\right)+f_{n+1} .
$$

Explicitly, $\mathcal{W}^{1,0}$ is generated by $\omega_{i}=s_{i}-z_{i} s_{n+1}, i=1, \ldots, n$. The sections $s_{i}$ for $i=2, \ldots, n+1$ are $T$-invariant and extend to $\widetilde{s_{i}}$ over the boundary of the quotient by $T$ of $\mathbb{B}_{\text {Siegel }}$. Together with $\widetilde{s_{1}}=s_{1}-z_{1} s_{n+1}$ they form a basis of the Deligne extension of the local system.

A basis of $\mathcal{T}_{Y}(-\log \Delta)$ near a point in $\Delta$ is given by $2 \pi i q \frac{\partial}{\partial q}, \frac{\partial}{\partial z_{2}}, \ldots, \frac{\partial}{\partial z_{n}}$. One calculates that the matrix of derivatives of the $\omega_{i}$ in these directions is minus the identity. This is equivalent to $\tau$ being an isomorphism near the boundary and to the vanishing of the cokernel, as claimed.

7.4. Computation of $c_{1}\left(\operatorname{coker} \tau^{\vee}\right)$ for the case $(\Sigma I N T)$. The calculations proceed analogously to the (INT) case with some minor twists.

Let $N \geq 5$, and let $f: \mathcal{X} \rightarrow B_{0}$ be a cyclic covering of type $\left(d, a_{1}, \ldots, a_{N}\right)$, and assume now that the collection $\mu_{i}=a_{i} / d$ satisfies (EINT) for some $S \subset\{1, \ldots, N\}$. Then $B / \Sigma=\mathbb{B}^{n} / \Gamma$ is a ball quotient. The primitive part of the VHS defined by $f$ then furnishes a VHS on $F / \Sigma$. Let $\mathbb{L}=\mathbb{L}_{k, \Sigma}$ be one of its direct summands, which is polarized by a hermitian form of signature $(1, n)$.

We choose again an appropriate subgroup $\Gamma^{\prime} \subset \Gamma$, and define $B^{u}, \pi, Y, \Delta$ and $\mathbb{L}^{u}$ analogously to the (INT)-case. Again the results of Section 7.1 apply; Let $\tau^{\vee}=\tau_{k}^{\vee}$ be the dual of the Higgs field, and let $p_{k, \Sigma}=p_{k, \Sigma}^{1}$ be the period map for $\mathbb{L}$. 
Let $F \subset B_{0}$ be the open dense submanifold, where $\Sigma$ acts freely, and let $M \subset$ $B_{0} \backslash F$ be the codimension one fix locus of $\Sigma$ in $B_{0}$ (which is only present in a few cases). We denote by $M_{l}, l=1, \ldots, r$ the irreducible components of the closure of $M$ in $B$. Let $\varpi: B \rightarrow B / \Sigma$ be the canonical projection, and let

$$
\left\{\bar{L}_{i j}\right\} \text { and }\left\{\bar{M}_{l}\right\}
$$

be a system of representatives of $\Sigma$-orbits of the divisors $L_{i j}$ and $M_{l}$.

Lemma 7.11 ([DM93, Lemma 8.3.2]). The map $\varpi: B \rightarrow B / \Sigma$ is ramified of order two at each smooth point of $\bigcup_{i, j \in S} L_{i j} \cup \bigcup_{l} M_{l}$.

Lemma 7.12. The ramification order of $\pi: B^{u} \rightarrow B / \Sigma$ at a point $x \in B^{u}$

$$
\begin{cases}\kappa_{i j} & , \text { for generic } x \text { in } \pi^{-1} \bar{L}_{i j} \text { with } i, j \notin S \\ 2 \kappa_{i j} & , \text { for generic } x \text { in } \pi^{-1} \bar{L}_{i j} \text { with } i, j \in S \\ 2 & , \text { for generic } x \in \pi^{-1} \bar{M}_{l} \\ 1 & , \text { if } x \in \pi^{-1}(\varpi(F))\end{cases}
$$

Moreover, we have

$$
\mathrm{c}_{1}\left(\operatorname{coker} \tau^{\vee}\right)=\sum_{i, j} n_{i j}^{k} \cdot \sum_{\substack{L \subset \pi^{-1} \bar{L}_{i j} \\ \text { irred. }}}[L]
$$

where the $n_{i j}^{k}$ are given as in Lemma 7.6.

Proof. a) Let $x$ be a point in $\varpi(F)$ or on one of the divisors $\bar{L}_{i j}$ or $\bar{M}_{l}$, but outside the intersection with any of the other divisors. The map $\varpi$ is locally at $x$ a ramified covering map. By Lemma 7.11, its multi-valued inverse is of the form

$$
\left(z_{1}, \ldots, z_{n}\right) \mapsto\left(z_{1}^{1 / 2}, z_{2}, \ldots, z_{n}\right) \quad \text { or } \quad \text { id }: \mathbb{C}^{n} \rightarrow \mathbb{C}^{n}
$$

depending on whether $x \in \bigcup_{i, j \in S} L_{i j} \cup \bigcup_{l} M_{l}$ or not. If $p_{k}$ denotes the multivalued period map associated with $\mathbb{L}_{k}$ on $B$, then $p_{k, \Sigma}$ is locally at $x$ given as the composition of the multi-valued inverse of $\varpi$ and $p_{k}$. For $k=1$, the single-valued period map is an isomorphism of $\widetilde{B}_{\Sigma}^{1}$ with $\mathbb{B}^{n}$, and the map $\mathbb{B}^{n} \rightarrow B^{u}$ is unramified, so $\pi: B^{u} \rightarrow B$ must precisely make up for the ramification of the multi-valued map $p_{1, \Sigma}$. This shows a).

b) First, notice that we can copy the proof of Lemma 7.5 to see that the support of $c_{1}\left(\operatorname{coker} \tau^{\vee}\right)$ is contained in the complement of $\pi^{-1}(\varpi(F))$. Next by essentially the same arguments as in the proof of Lemma 7.10, there are no contributions from the boundary $\Delta$. Furthermore, $n_{i j}^{k}$ is again given by the vanishing order of $\operatorname{det}\left(J\left(p_{k, \Sigma}\right)\right)$. By the proof of Part a) and the chain rule, this vanishing order is still given as in Lemma 7.6.

7.5. Collecting the contributions. In this section, we gather all the steps needed to compute the Lyapunov exponents, and thus to prove Theorem 7.1

Since (IINT) comprises (INT), we consider the setup of Section 7.4, and work with the notations introduced there. In particular, $N \geq 5, \mathbb{L}=\mathbb{L}_{k, \Sigma}$ is a direct summand of the primitive part of the VHS on $B / \Sigma$, induced by a cyclic covering, and $\tau^{\vee}$ is the dual Higgs field of the pullback VHS $\mathbb{L}^{u}$ on $B^{u}$.

Proposition 7.13. The positive Lyapunov spectrum of $\mathbb{L}^{u}$ is

$$
\lambda_{1}=\lambda_{1} \geq \underbrace{0=\cdots=0}_{n-1}
$$


where

$$
\lambda_{1}=1-\frac{\mathrm{c}_{1}\left(\operatorname{coker} \tau^{\vee}\right) \cdot \mathrm{c}_{1}\left(\omega_{Y}\right)^{n-1}}{\mathrm{c}_{1}\left(\omega_{Y}\right)^{n}} .
$$

Proof. By Theorem1.1 1ii), $2 n-2$ of the $2 n+2$ Lyapunov exponents of $\mathbb{L}^{u}$ are zero. Moreover, since we are working with a $\mathbb{C}$-variation, the first Lyapunov exponent occurs with multiplicity 2 , i.e.

$$
\lambda_{1}=\lambda_{2} .
$$

By Proposition 3.2 and its proof, the Lyapunov spectrum of $\mathbb{W}_{\mathbb{R}}$, the $\mathbb{R}$-variation associated with $\mathbb{L}_{u} \oplus \overline{\mathbb{L}_{u}}$, is the same as the one of $\mathbb{L}^{u}$. Therefore, by Theorem 1.1 iv),

$$
2 \lambda_{1}=\frac{(n+1) \mathrm{c}_{1}\left(\mathcal{W}^{1,0}\right) \cdot \mathrm{c}_{1}\left(\omega_{Y}\right)^{n-1}}{\mathrm{c}_{1}\left(\omega_{Y}\right)^{n}}
$$

with $\mathcal{W}^{1,0}=\mathcal{E}^{1,0} \oplus \overline{\mathcal{E}}^{1,0}$, where $\mathcal{E}^{1,0}$ and $\overline{\mathcal{E}}^{1,0}$ are the 1,0 -parts of the Deligne extensions of the holomorphic subbundles associated with $\mathbb{L}^{u}$ and $\overline{\mathbb{L}^{u}}$. From the antiholomorphic isomorphism $\mathcal{E}^{0,1} \cong \overline{\mathcal{E}}^{1,0}$ and the fact that $\mathrm{c}_{1}\left(\mathcal{E}^{1,0}\right)=-\mathrm{c}_{1}\left(\mathcal{E}^{0,1}\right)$, we infer that

$$
\mathrm{c}_{1}\left(\mathcal{W}^{1,0}\right)=2 \mathrm{c}_{1}\left(\mathcal{E}^{1,0}\right)
$$

Now, Lemma 7.3 implies the claim.

Next, we argue that we can compute the above intersection products on $B / \Sigma$ instead of $B^{u}$. We introduce the divisor $D_{k} \in \mathrm{CH}_{1}\left(B^{\mathrm{nc}} / \Sigma\right)_{\mathbb{Q}}$ given by

$$
D_{k, \Sigma}=\sum_{i, j \in S} \frac{n_{i j}^{k}}{2 \kappa_{i j}}\left[\bar{L}_{i j}\right]+\sum_{i, j \notin S} \frac{n_{i j}^{k}}{\kappa_{i j}}\left[\bar{L}_{i j}\right]
$$

and the orbifold canonical divisor

$$
K_{\Sigma}^{\mathrm{orb}}=K_{B^{\mathrm{nc}} / \Sigma}+\sum_{i, j \in S}\left(1-\frac{1}{2 \kappa_{i j}}\right)\left[\bar{L}_{i j}\right]+\sum_{i, j \notin S}\left(1-\frac{1}{\kappa_{i j}}\right)\left[\bar{L}_{i j}\right]+\sum_{l} \frac{1}{2}\left[\bar{M}_{l}\right]
$$

with $n_{i j}^{k}$ from Lemma $7.6, \kappa_{i j}=\left(1-\mu_{i}-\mu_{j}\right)^{-1}$ and the convention $1 / \kappa_{i j}=0$, if $\mu_{i}+\mu_{j}=1$. We also define the divisors

$$
D_{k}=\sum_{\substack{i, j \\ \mu_{i}+\mu_{j}<1}} \frac{n_{i j}^{k}}{\kappa_{i j}}\left[L_{i j}\right] \quad \text { and } \quad K^{\text {orb }}=K_{B}+\sum_{\substack{i, j \\ \mu_{i}+\mu_{j} \leq 1}}\left(1-\frac{1}{\kappa_{i j}}\right)\left[L_{i j}\right]
$$

upstairs in $\mathrm{CH}_{1}\left(B^{\text {nc }}\right)_{\mathbb{Q}}$.

Lemma 7.14. We have

$$
\lambda_{1}=1-\frac{\mathrm{c}_{1}\left(\operatorname{coker} \tau^{\vee}\right) \cdot \mathrm{c}_{1}\left(\omega_{Y}\right)^{n-1}}{\mathrm{c}_{1}\left(\omega_{Y}\right)^{n}}=1-\frac{D_{k, \Sigma} \cdot\left(K_{\Sigma}^{\mathrm{orb}}\right)^{n-1}}{\left(K_{\Sigma}^{\mathrm{orb}}\right)^{n}}=1-\frac{D_{k} \cdot\left(K^{\mathrm{orb}}\right)^{n-1}}{\left(K^{\mathrm{orb}}\right)^{n}} .
$$

Proof. By Lemma 7.12, the divisors $D_{k}$ and $K^{\text {orb }}$ are adapted to satisfy

$$
\pi^{*} D_{k, \Sigma}=\mathrm{c}_{1}\left(\operatorname{coker} \tau^{\vee}\right) \text { and } \pi^{*} K_{\Sigma}^{\mathrm{orb}}=\mathrm{c}_{1}\left(\omega_{Y}\right),
$$

and by Lemma 7.11,

$$
\varpi^{*} D_{k, \Sigma}=D_{k} \quad \text { and } \quad \varpi^{*} K_{\Sigma}^{\mathrm{orb}}=K^{\mathrm{orb}} .
$$

The ring structure on $\mathrm{CH}_{*}\left(B^{\mathrm{nc}} / \Sigma\right)_{\mathbb{Q}}$ does not depend on the presentation of $B^{\text {nc }} / \Sigma$ as a finite quotient, whence

$$
(1 / \operatorname{deg}(\pi)) \pi^{*} D_{1} \cdot \pi^{*} D_{2}=D_{1} \cdot D_{2}=(1 /|\Sigma|) \varpi^{*} D_{1} \cdot \varpi^{*} D_{2}
$$


for any $D_{1}, D_{2}$ in $\mathrm{CH}_{*}\left(B^{\mathrm{nc}} / \Sigma\right)_{\mathbb{Q}}$.

We can now plug in the concrete realizations of $\mathrm{CH}_{*}\left(B^{\mathrm{nc}}\right)_{\mathbb{Q}}$ from Section 6.4 to finish the computation in each individual case.

\section{ORBIFold Euler nUmbers AND LOG-BALl QUOTIENTS}

Thurston considers in his paper [Thu98] the moduli space $C\left(\mu_{1}, \ldots, \mu_{N}\right)$ of Euclidean metrics on the sphere of total area one with conical singularities at $N$ points of fixed angles $\left(2 \pi \mu_{1}, \ldots, 2 \pi \mu_{N}\right)$ (where $\mu_{i} \in \mathbb{Q} \cap(0,1)$ ). If the sum of angles $\sum \mu_{i}=2$, Thurston provides this space with a hyperbolic metric so that, up to taking the quotient by the finite group permuting the points of equal angle, the metric completion $\bar{C}\left(\mu_{1}, \ldots, \mu_{N}\right)$ of this moduli space is the space of $\left(\mu_{i}\right)_{i}$-stable points $B^{\mu}$. This metric completion is a hyperbolic cone manifold, and an orbifold ball quotient precisely when ( $(\Sigma I N T)$ is satisfied.

For each of these hyperbolic cone manifolds, one can study its volume. In particular, for $\mu=\left(\mu_{i}(1)\right)_{i}$ parametrizing an orbifold ball quotient and $\mu(k)$ a Galois conjugate tuple thereof with $\sum_{i} \mu_{i}(k)=2$, the ratio of the volumes of $C\left(\mu_{1}(k), \ldots, \mu_{N}(k)\right)$ and $C\left(\mu_{1}(1), \ldots, \mu_{N}(1)\right)$ is an interesting invariant, which remains unchanged under passage to a covering defined by a finite index subgroup of the associated lattice.

Here we study the corresponding algebro-geometric version of this invariant, the orbifold Euler number. By the Gauß-Bonnet theorem it is proportional to the volume of the hyperbolic cone manifold.

Since all but one of the known non-arithmetic lattices are in dimension at most two and for simplicity we restrict to the surface case. The orbifold Euler number, as introduced by Langer Lan03, is defined for any pair $(X, D)$ of a normal projective surface and a $\mathbb{Q}$-Divisor $D=\sum a_{i} D_{i}$, where the $D_{i}$ are prime divisors and $0 \leq$ $a_{i} \leq 1$, and is given by

$$
\mathrm{e}^{\text {orb }}(X, D)=\mathrm{e}^{\mathrm{top}}(X)-\sum a_{i} \mathrm{e}^{\mathrm{top}}\left(D_{i} \backslash \operatorname{Sing}(X, D)\right)-\sum_{x \in \operatorname{Sing}(X, D)} 1-\mathrm{e}^{\text {orb }}(x, X, D) .
$$

It extends the well-studied case when $a_{i}=(1-1 / m)$ with $m \in \mathbb{N}$, i. e. when $(X, D)$ is an actual orbifold. Here, $\operatorname{Sing}(X, D)$ is the locus where either $X$ or $\operatorname{supp} D$ is singular, and $\mathrm{e}^{\text {orb }}(x, X, D)$ is the local orbifold Euler number that we will give below in the cases of interest.

Fix a quintuple $\left(\mu_{i}\right)_{i=1}^{5}$. First, we only suppose that $\sum_{i} \mu_{i}=2$ and consider the space $B^{\mu, \mathrm{nc}}$ of $\mu$-semistable points, blown up at the cusps, together with the divisor

$$
R^{\mu}=\sum_{i<j: \mu_{i}+\mu_{j} \leq 1}\left(\mu_{i}+\mu_{j}\right)\left[L_{i j}\right] .
$$

Further let $K_{B^{\mu, \text { nc }}}$ denote the canonical divisor class of $B^{\mu, \mathrm{nc}}$.

Theorem 8.1. For any $\left(\mu_{i}\right)_{i=1}^{5} \in(\mathbb{Q} \cap(0,1))^{5}$ with $\sum_{i} \mu_{i}=2$, the pair $\left(B^{\mu, \mathrm{nc}}, R^{\mu}\right)$ is a is a log-ball quotient in the sense that with the definition $\mathrm{c}_{1}^{\text {orb }}\left(B^{\mu, \mathrm{nc}}, R^{\mu}\right)=$ $K_{B^{\mu, \mathrm{nc}}}+R^{\mu}$ equality is attained in the generalized Bogomolov-Miyaoka-Yau inequality, i.e.

$$
3 \mathrm{e}^{\mathrm{orb}}\left(B^{\mu, \mathrm{nc}}, R^{\mu}\right)=\left(\mathrm{c}_{1}^{\mathrm{orb}}\left(B^{\mu, \mathrm{nc}}, R^{\mu}\right)\right)^{2}
$$


It is classically known that 2-dimensional ball quotients are the surfaces of general type realizing equality in the Bogomolov-Miyaoka-Yau inequality $3 \mathrm{c}_{2} \geq \mathrm{c}_{1}^{2}$. This inequality can be generalized to orbifolds and further to $\log$-canonical pairs $(X, D)$ (see Lan03 and references therein). The BMY-equality of orbifold Chern classes has been used to find new examples of ball quotients (for an account see Yos87, $\S 10]$ ). Our theorem yields new examples of log-varieties satisfying a BMY-equality without being a ball quotient.

In the case that $\mu$ satisfies (INT), the orbifold canonical divisor $c_{1}^{\text {orb }}\left(\mathcal{B}^{\ell}, \mathcal{R}^{\ell}\right)$ coincides with $K^{\text {orb }}$ in (25) and we recover the orbifold version of Chern class proportionality on a ball quotient.

From this theorem we will deduce that the relative orbifold Euler characteristics are ratios of intersection numbers on the ball quotient and thus commensurability invariants by Corollary 4.5. For the precise statement, let us fix a quintuple

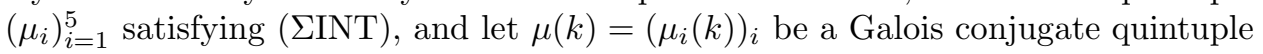
parametrizing a local system of signature $(1,2)$. For $\ell \in\{1, k\}$, we consider the space $\mathcal{B}^{\ell}=B^{\text {nc, }, \mu(\ell)} / \Sigma$ and define the divisor $\mathcal{R}^{\ell}$ to be the image of $R^{\mu(\ell)}$ in the Chow ring of $\mathcal{B}^{\ell}$.

Corollary 8.2. Suppose $\left(\mu_{i}\right)_{i=1}^{5}$ satisfies (EINT), and let $\mu(k)=\left(\mu_{i}(k)\right)_{i}$ be a Galois conjugate quintuple parametrizing a local system of signature $(1,2)$. Then

$$
\frac{\mathrm{e}^{\mathrm{orb}}\left(\mathcal{B}^{k}, \mathcal{R}^{k}\right)}{\mathrm{e}^{\mathrm{orb}}\left(\mathcal{B}^{1}, \mathcal{R}^{1}\right)}=\frac{\left(K^{\text {orb }}-D_{k}\right)^{2}}{\left(K^{\text {orb }}\right)^{2}}=\frac{\mathrm{c}_{1}\left(\mathcal{H o m}\left(\mathcal{E}_{k}^{1,0}, \mathcal{E}_{k}^{0,1}\right)\right)^{2}}{\mathrm{c}_{1}\left(\omega_{Y}\right)^{2}}=\frac{9 \mathrm{c}_{1}\left(\mathcal{E}_{k}^{1,0}\right)^{2}}{\mathrm{c}_{1}\left(\omega_{Y}\right)^{2}}
$$

where $Y$ is as in Section 7.4 and $\mathcal{E}_{k}^{1,0}$ (resp. $\mathcal{E}_{k}^{0,1}$ ) is the holomorphic subbundle (quotient bundle) of the pullback VHS of $\mathbb{L}_{k}$.

Using the structure of the Chow ring explicated in Section 6.4.1, we can evaluate the right hand side of the (28). We can also evaluate the left hand side explicitly.

Proposition 8.3. The orbifold Euler number of $\left(B^{\mu, \mathrm{nc}}, R^{\mu}\right)$ is

$$
\begin{aligned}
\mathrm{e}^{\text {orb }}\left(B^{\mu, \mathrm{nc}}, R^{\mu}\right)= & \left.7+\sum_{\substack{i<j: \mu_{i}+\mu_{j} \leq 1\\
}}\left(\mu_{i}+\mu_{j}\right)+\sum_{\substack{i<j: \mu_{i}+\mu_{j}>1 \\
i<j, l<m: \\
i<j \neq m \\
\mu_{i}+\mu_{j} \leq 1 \\
\mu_{l}+\mu_{m} \leq 1}}\left(1-\mu_{i}-\mu_{j}\right)\left(1-\mu_{l}-\mu_{m}\right)-1\right)^{2}-2
\end{aligned}
$$

We will combine these two results for the proof of Theorem 8.1. It would however be interesting to find a more conceptual explanation for the proportionality relation, using the fact that on the open dense set $\widetilde{B}_{0}$ in the Fox completion $\widetilde{B}^{\mu}$, the period map is étale and induces a sheaf isomorphism of $\Omega_{B_{0}}^{1}$ with $\mathcal{H o m}\left(\mathcal{L}^{1,0}, \mathcal{L}^{0,1}\right)$, the sheaf induced by pulling back the cotangent sheaf of $\mathbb{B}^{n}$. The major difficulty seems to be the lack of a complex structure on the Fox completion for arbitrary $\mu$ with $\sum_{i} \mu_{i}=2$.

8.1. Orbifold Euler numbers. For our computations it will be sufficient to know $\mathrm{e}^{\text {orb }}(x, X, D)$ when $X$ is smooth and $D$ has normal crossings or ordinary triple points, i.e. locally at $x \in \operatorname{Sing}(D)$ the divisor $D$ is analytically isomorphic to $\sum_{i=1}^{n} a_{i} L_{i}$ where $L_{i}$ are $n \leq 3$ distinct lines in $\mathbb{C}^{2}$ passing through the origin. 
Then $\mathrm{e}^{\text {orb }}(x, X, D)=\mathrm{e}^{\text {orb }}\left(0, \mathbb{C}^{2}, \sum a_{i} L_{i}\right)$. In this situation, we have in particular Lan03, Theorem 8.3]

$$
\mathrm{e}^{\text {orb }}\left(0, \mathbb{C}^{2}, \sum a_{i} L\right)= \begin{cases}\left(1-a_{1}\right) \cdot\left(1-a_{2}\right), & \text { if } n=2 \\ \frac{1}{4} \cdot\left(a_{1}+a_{2}+a_{3}-2\right)^{2}, & \text { if } n=3, a_{1} \leq a_{2} \leq a_{3}, \\ & a_{3}<a_{1}+a_{2} \text { and } \\ & a_{1}+a_{2}+a_{3} \leq 2 .\end{cases}
$$

Another property of $\mathrm{e}^{\text {orb }}$ that we will use is the fact that it behaves multiplicatively under pullback, i.e if $f: Y \rightarrow X$ is a finite, proper morphism of normal, proper surfaces and $f^{*}\left(K_{X}+D\right)=K_{Y}+D^{\prime}$ for a $\mathbb{Q}$-divisor $D^{\prime}$, then

$$
\mathrm{e}^{\text {orb }}\left(Y, D^{\prime}\right)=\operatorname{deg}(f) \cdot \mathrm{e}^{\text {orb }}(X, D) \text {. }
$$

We can now prove the explicit formula for the orbifold Euler number of the pair $\left(B^{\mu, \mathrm{nc}}, R^{\mu}\right)$.

Proof of Proposition 8.3. The topological Euler characteristic of $B^{\mu, n c}$ is 7 minus the number of contracted $L_{i j}$ 's. $L_{i j}$ is contracted whenever $\mu_{i}+\mu_{j}>1$, whence the third summand has a $(-2)$ instead of the $(-1)$ appearing in (26). The second summand is the codimension 1 contribution. The coefficient of $L_{i j}$, whenever present in $R^{\mu}$, is $a_{i j}=\mu_{i}+\mu_{j}$, and $L_{i j} \backslash \operatorname{Sing}\left(R^{\mu}\right)$ is a thrice-punctured $\mathbb{P}^{1}$. The remaining summands are the codimension 2 contributions. For each contracted $L_{i j}$ we have an ordinary triple point with contribution

$$
\frac{1}{4}\left(a_{l m}+a_{l q}+a_{m q}-2\right)^{2}=\left(\mu_{l}+\mu_{m}+\mu_{q}-1\right)^{2}=\left(\mu_{i}+\mu_{j}-1\right)^{2},
$$

where $\{l, m, q\}=\{1, \ldots, 5\} \backslash\{i, j\}$. Note that $a_{\sigma(l) \sigma(m)} \leq a_{\sigma(l) \sigma(q)}+a_{\sigma(m) \sigma(q)}$ for every permutation $\sigma$ of $\{l, m, q\}$ and $a_{l m}+a_{l q}+a_{m q}<2$. The last summand is the contribution from the ordinary double points.

8.2. Proof of Theorem $\mathbf{8 . 1}$ and Corollary $\mathbf{8 . 2}$, The space $B^{\mu, n c}$ results from $B_{10}$ by blowing down all the divisors $L_{i j}$ with $\mu_{i}+\mu_{j}>1$. Before engaging in the proof of Theorem 8.1, we compute the pullback of the "boundary" divisor $R^{\mu}$ under this blowup map.

Lemma 8.4. Let $\mu \in(\mathbb{Q} \cap(0,1))^{5}, \sum_{i} \mu_{i}=2$ and let $b: B_{10} \rightarrow B^{\mu, \mathrm{nc}}$ denote the map blowing down all divisors $L_{i j}$ with $\mu_{i}+\mu_{j}>1$. Then

$$
b^{*}\left(K_{B^{\mu, \mathrm{nc}}}+R^{\mu}\right)=K_{B_{10}}+\sum_{\substack{i<j: \\ \mu_{i}+\mu_{j} \leq 1}}\left(\mu_{i}+\mu_{j}\right)\left[L_{i j}\right]+\sum_{\substack{i<j: \\ \mu_{i}+\mu_{j}>1}}\left(3-2\left(\mu_{i}+\mu_{j}\right)\right)\left[L_{i j}\right] .
$$

Proof. Since $b$ is a composition of several blowdowns that commute with each other, we can treat each blowdown individually. So assume first that there is only one pair $\{a, b\} \subset\{1, \ldots, 5\}$ such that $\mu_{a}+\mu_{b}>1$. The image of the exceptional divisor $L_{a b}$ under $b$ is the intersection of the lines $L_{c d}^{\mu}, L_{c e}^{\mu}$ and $L_{d e}^{\mu}$ in $B^{\mu}$, where $\{a, b, c, d, e\}=\{1, \ldots, 5\}$. The total transform of a boundary divisor $\left[L_{i j}^{\mu}\right]$ is thus

$$
b^{*}\left[L_{i j}^{\mu}\right]= \begin{cases}{\left[L_{i j}\right]+\left[L_{a b}\right],} & \text { if }\{i, j\} \subset\{c, d, e\} \\ {\left[L_{i j}\right],} & \text { otherwise }\end{cases}
$$


Therefore, we obtain altogether

$$
\begin{aligned}
b^{*}\left(K_{B^{\mu, \mathrm{nc}}}+R^{\mu}\right)=K_{B_{10}}+\sum_{\substack{i<j: \\
\mu_{i}+\mu_{j} \leq 1}}\left(\mu_{i}+\mu_{j}\right)\left[L_{i j}\right] \\
+\sum_{\substack{a<b: \\
\mu_{a}+\mu_{b}>1 \\
\{a, b, c, d, e\}=\{1, \ldots, 5\}}}\left(-1+2\left(\mu_{c}+\mu_{d}+\mu_{e}\right)\right)\left[L_{a b}\right] .
\end{aligned}
$$

Using $\sum_{i} \mu_{i}=2$, we obtain the claim.

Proof of Theorem 8.1. We first show the identity $\left(K_{B^{\mu}}+R^{\mu}\right)^{2}=\mathrm{e}^{\text {orb }}\left(B^{\mu}, R^{\mu}\right)$ for a configuration of type $B_{10}$. We have $K_{B_{10}}^{2}=5$, and $\left[L_{i j}\right] \cdot K_{B_{10}}=-1$. Moreover

$$
\left[L_{i j}\right] \cdot\left[L_{l m}\right]= \begin{cases}0, & \text { if }|\{i, j\} \cap\{l, m\}|=1 \\ 1, & \text { if }\{i, j\} \cap\{l, m\}=\emptyset \\ -1, & \text { if }\{i, j\}=\{l, m\}\end{cases}
$$

Hence,

$$
\begin{aligned}
\left(\sum_{i<j}\left(\mu_{i}+\mu_{j}\right)\left[L_{i j}\right]\right)^{2} & =-\sum_{i<j}\left(\mu_{i}+\mu_{j}\right)^{2}+2 \cdot \sum_{i<j, l<m, i<l}\left(\mu_{i}+\mu_{j}\right)\left(\mu_{l}+\mu_{m}\right) \\
& =-4 \sum_{i} \mu_{i}^{2}-2 \sum_{i<j} \mu_{i} \mu_{j}+12 \sum_{i<j} \mu_{i} \mu_{j}=18 \sum_{i<j} \mu_{i} \mu_{j}-16,
\end{aligned}
$$

where we use $4=\left(\sum_{i} \mu_{i}\right)^{2}=\sum_{i} \mu_{i}^{2}+2 \sum_{i<j} \mu_{i} \mu_{j}$. Altogether we obtain

$$
\begin{aligned}
\left(K_{B_{10}}+\sum_{i<j}\left(\mu_{i}+\mu_{j}\right)\left[L_{i j}\right]\right)^{2} & =K_{B_{10}}^{2}+2 K_{B_{10}} \cdot\left(\sum_{i<j}\left(\mu_{i}+\mu_{j}\right)\left[L_{i j}\right]\right)+\left(\sum_{i<j}\left(\mu_{i}+\mu_{j}\right)\left[L_{i j}\right]\right)^{2} \\
& =5+(-2) \cdot 4 \sum_{i} \mu_{i}+18 \sum_{i<j} \mu_{i} \mu_{j}-16 \\
& =18 \sum_{i<j} \mu_{i} \mu_{j}-27 .
\end{aligned}
$$

On the other hand,

$$
\begin{aligned}
\mathrm{e}^{\mathrm{orb}}\left(B_{10}, R^{\mu}\right) & =7+4 \cdot \sum_{i} \mu_{i}-15+\sum_{i<j, l<m, i<l}\left(1-\mu_{i}-\mu_{j}\right)\left(1-\mu_{l}-\mu_{m}\right) \\
& =15-\frac{4 !}{2} \sum_{i} \mu_{i}+\sum_{i<j, l<m, i<l}\left(\mu_{i}+\mu_{j}\right)\left(\mu_{l}+\mu_{m}\right) \\
& =-9+6 \sum_{i<j} \mu_{i} \mu_{j}
\end{aligned}
$$

Now let $\mu$ be arbitrary. By Lemma 8.4 , the blowdown map $b: B_{10} \rightarrow B^{\mu}$ satisfies

$$
b^{*}\left(K_{B^{\mu}}+R^{\mu}\right)=\overbrace{K_{B_{10}}+\sum_{i<j}\left(\mu_{i}+\mu_{j}\right)\left[L_{i j}\right]}^{A}+\overbrace{\sum_{\substack{i<j: \\ \mu_{i}+\mu_{j}>1}} 3\left(1-\mu_{i}-\mu_{j}\right)\left[L_{i j}\right]}^{B} .
$$

We proceed by computing the individual summands of

$$
\left(K_{B^{\mu}}+R^{\mu}\right)^{2}=\left(b^{*}\left(K_{B^{\mu}}+R^{\mu}\right)\right)^{2}=A^{2}+2 A B+B^{2} .
$$


For $i<j$ with $\mu_{i}+\mu_{j}>1$, let $\{l, m, q\}$ be the complement in $\{1, \ldots, 5\}$. Then

$$
\begin{aligned}
\left.\left(K_{B_{10}}+\sum_{i^{\prime}<j^{\prime}}\left(\mu_{i^{\prime}}+\mu_{j^{\prime}}\right)\left[L_{i^{\prime} j^{\prime}}\right]\right) \cdot\left[L_{i j}\right]\right) & =-1+2\left(\mu_{l}+\mu_{m}+\mu_{q}\right)-\left(\mu_{i}+\mu_{j}\right) \\
& =3\left(1-\mu_{i}-\mu_{j}\right)
\end{aligned}
$$

As different $L_{i j}$ 's with $\mu_{i}+\mu_{j}>1$ do not intersect,

$$
\left(\sum_{\substack{i<j: \\ \mu_{i}+\mu_{j}>1}} 3\left(1-\mu_{i}-\mu_{j}\right)\left[L_{i j}\right]\right)^{2}=-9 \sum_{\substack{i<j: \\ \mu_{i}+\mu_{j}>1}}\left(1-\mu_{i}-\mu_{j}\right)^{2}
$$

Putting everything together, using that the identity $A^{2}=18 \sum_{i<j} \mu_{i} \mu_{j}-27$ also holds for arbitrary $\mu$, we obtain

$$
\begin{aligned}
\left(K_{B^{\mu}}+R^{\mu}\right)^{2} & =\left(K_{B_{10}}+\sum_{i<j}\left(\mu_{i}+\mu_{j}\right) L_{i j}\right)^{2}+9 \sum_{\substack{i<j: \\
\mu_{i}+\mu_{j}>1}}\left(1-\mu_{i}-\mu_{j}\right)^{2} \\
& =18 \sum_{i<j} \mu_{i} \mu_{j}-27+9 \sum_{\substack{i<j: \\
\mu_{i}+\mu_{j}>1}}\left(1-\mu_{i}-\mu_{j}\right)^{2} .
\end{aligned}
$$

Now we investigate the amount by which the Euler characteristic changes for each $i<j$ with $\mu_{i}+\mu_{j}>1$. First observe that we still can evaluate the right hand side of (29) for the boundary divisor $\sum_{i<j}\left(\mu_{i}+\mu_{j}\right)\left[L_{i j}\right]$ on $B_{10}$ and that $\mathrm{e}^{\text {orb }}\left(B^{\mu}, R^{\mu}\right)$ is off this quantity by some "error term" $M$

$$
\mathrm{e}^{\mathrm{orb}}\left(B^{\mu}, R^{\mu}\right)=6 \sum_{i<j} \mu_{i} \mu_{j}-9+M
$$

with $M$ being the sum over all $i<j$ with $\mu_{i}+\mu_{j}>1$ of the expression

$$
-\left(\mu_{i}+\mu_{j}\right)+\left(\mu_{i}+\mu_{j}-1\right)^{2}-2-\left(1-\mu_{i}-\mu_{j}\right)\left(3-2\left(\mu_{l}+\mu_{m}+\mu_{q}\right)\right)+3,
$$

where $\{l, m, q\}$ is again a complement of $\{i, j\}$. Evaluating this yields

$$
M=3 \sum_{\substack{i<j: \\ \mu_{i}+\mu_{j}>1}}\left(1-\mu_{i}-\mu_{j}\right)^{2},
$$

which altogether implies $3 \mathrm{e}^{\mathrm{orb}}\left(B^{\mu}, R^{\mu}\right)=\left(K_{B^{\mu}}+R^{\mu}\right)^{2}$.

The starting point for the proof of Corollary 8.2 is that for $\mu=\mu(1)$ satisfying

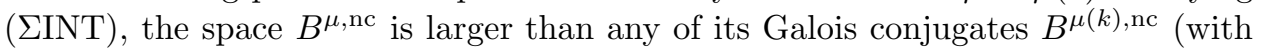
$\left.\sum_{i} \mu_{i}(k)=2\right)$ in the sense that $B^{\mu, \mathrm{nc}}$ is an intermediate space in the chain of blowdowns $B_{10} \rightarrow \cdots \rightarrow B^{\mu(k), \text { nc }}$. More precisely,

Lemma 8.5. Suppose $\mu \in(\mathbb{Q} \cap(0,1))^{5}$ satisfies (LINT), and let $\mu(k)$ be a Galois conjugate of signature $(1,2)$.

a) For any pair $\{i, j\} \subset\{1, \ldots, 5\}, \mu_{i}+\mu_{j}>1$ implies $\mu_{i}(k)+\mu_{j}(k)>1$.

In particular, there is a blowdown map $b: B^{\mu, \mathrm{nc}} \rightarrow B^{\mu(k), \mathrm{nc}}$ contracting some of the boundary divisors $L_{i j}$ with self-intersection $(-1)$.

b) $b^{*}\left(K_{B^{\mu(k), \mathrm{nc}}}+R^{\mu(k)}\right)=K^{\text {orb }}-D_{k}$. 
Proof. a) One checks all the finitely many cases. b) By the proof of Lemma 8.4, we find that the coefficient of $b^{*}\left(K_{B^{\mu(k), n c}}+R^{\mu(k)}\right)$ in front of $\left[L_{i j}\right]$ is

$$
3-2\left(\mu_{i}(k)+\mu_{j}(k)\right)=1-\frac{n_{i j}^{k}}{\kappa_{i j}} .
$$

This property is not true for arbitrary $\mu$ with $\sum_{i} \mu_{i}=2$ and we suspect it to reflect a metric contraction property of the non-uniformizing period maps relative to the uniformizing one, analogously to the case of Teichmüller curves.

Proof of Corollary 8.2. First, note that

$$
\frac{\mathrm{e}^{\text {orb }}\left(\mathcal{B}^{k}, \mathcal{R}^{k}\right)}{\mathrm{e}^{\text {orb }}\left(\mathcal{B}^{1}, \mathcal{R}^{1}\right)}=\frac{\mathrm{e}^{\text {orb }}\left(B^{\mu(k), \mathrm{nc}}, \mathcal{R}^{\mu(k)}\right)}{\mathrm{e}^{\text {orb }}\left(B^{\mu, \mathrm{nc}}, R^{\mu}\right)}
$$

using the multiplicative behavior of $\mathrm{e}^{\text {orb }}$ under pullback. The first equality reduces thus to combining Theorem 8.1 with Lemma $8.5 \mathrm{~b}$ ).

For the second and third equality, let again $\pi: Y \rightarrow \mathcal{B}^{1}$ denote the extension of the canonical projection $B^{u}=\mathbb{B}^{2} / \Gamma^{\prime} \rightarrow B^{1} / \Sigma$ as in Section 7.4. Then by (23) and using $\mathrm{c}_{1}\left(\mathcal{E}^{1,0}\right)=-\mathrm{c}_{1}\left(\mathcal{E}^{0,1}\right)$, we obtain

$$
\begin{aligned}
\pi^{*}\left(K^{\text {orb }}-D_{k}\right) & =\mathrm{c}_{1}\left(\omega_{Y}\right)-\mathrm{c}_{1}(\operatorname{coker} \widetilde{\tau}) \\
& =-\mathrm{c}_{1}\left(\mathcal{H o m}\left(\mathcal{E}^{1,0}, \mathcal{E}^{0,1}\right)\right) \\
& =-\mathrm{c}_{1}\left(\left(\mathcal{E}^{1,0}\right)^{*} \otimes \mathcal{E}^{0,1}\right)=3 \mathrm{c}_{1}\left(\mathcal{E}^{1,0}\right)
\end{aligned}
$$

\section{REFERENCES}

[BM10] I. Bouw and M. Möller. Teichmüller curves, triangle groups, and Lyapunov exponents. Ann. of Math. (2), 172(1):139-185, 2010.

[Bou01] I. Bouw. The p-rank of ramified covers of curves. Compositio Math., 126(3):295-322, 2001.

[Bou05] I. Bouw. Pseudo-elliptic bundles, deformation data and the reduction of Galois covers, 2005 .

[CKS86] Eduardo Cattani, Aroldo Kaplan, and Wilfried Schmid. Degeneration of Hodge structures. Ann. of Math. (2), 123(3):457-535, 1986.

[CMSP03] J. Carlson, S. Müller-Stach, and C. Peters. Period mappings and period domains, volume 85 of Cambridge Studies in Advanced Mathematics. Cambridge University Press, Cambridge, 2003.

[CW93] P. B. Cohen and J. Wolfart. Fonctions hypergéométriques en plusieurs variables et espaces des modules de variétés abéliennes. Ann. Sci. École Norm. Sup. (4), 26(6):665690, 1993.

[Del70] P. Deligne. Équations différentielles à points singuliers réguliers. Lecture Notes in Mathematics, Vol. 163. Springer-Verlag, Berlin, 1970.

[Del87] P. Deligne. Un théorème de finitude pour la monodromie. In Discrete groups in geometry and analysis (New Haven, Conn., 1984), volume 67 of Progr. Math., pages 1-19. Birkhäuser Boston, Boston, MA, 1987.

[DM86] P. Deligne and G. D. Mostow. Monodromy of hypergeometric functions and nonlattice integral monodromy. Inst. Hautes Études Sci. Publ. Math., (63):5-89, 1986.

[DM93] P. Deligne and G. D. Mostow. Commensurabilities among lattices in $\mathrm{PU}(1, n)$, volume 132 of Annals of Mathematics Studies. Princeton University Press, Princeton, NJ, 1993.

[DPP11] M. Deraux, J. Parker, and J. Paupert. Census of the complex hyperbolic sporadic triangle groups. Exp. Math., 20(4):467-486, 2011. 
[DPP14] M. Deraux, J. Parker, and J. Paupert. New non-arithmetic complex hyperbolic lattices, 2014, arXiv:math.GT/1401.0308.

[EKZ11] Alex Eskin, Maxim Kontsevich, and Anton Zorich. Lyapunov spectrum of square-tiled cyclic covers. J. Mod. Dyn., 5(2):319-353, 2011.

[EKZ14] Alex Eskin, Maxim Kontsevich, and Anton Zorich. Sum of Lyapunov exponents of the Hodge bundle with respect to the Teichmüller geodesic flow. Publ. Math. Inst. Hautes Études Sci., 120:207-333, 2014.

[EV02] Hélène Esnault and Eckart Viehweg. Chern classes of Gauss-Manin bundles of weight 1 vanish. $K$-Theory, 26(3):287-305, 2002.

[FMZ14a] Giovanni Forni, Carlos Matheus, and Anton Zorich. Lyapunov spectrum of invariant subbundles of the Hodge bundle. Ergodic Theory Dynam. Systems, 34(2):353-408, 2014.

[FMZ14b] Giovanni Forni, Carlos Matheus, and Anton Zorich. Zero Lyapunov exponents of the Hodge bundle. Comment. Math. Helv., 89(2):489-535, 2014.

[For02] G. Forni. Deviation of ergodic averages for area-preserving flows on surfaces of higher genus. Ann. of Math. (2), 155(1):1-103, 2002.

[Ful84] W. Fulton. Intersection theory, volume 2 of Ergebnisse der Mathematik und ihrer Grenzgebiete (3) [Results in Mathematics and Related Areas (3)]. Springer-Verlag, Berlin, 1984.

[GH14] J. Grivaux and P. Hubert. Les exposants de Liapounoff du flot de Teichmüller [d'aprs Eskin-Kontsevich-Zorich]). Astérisque, (353):Exp. No. 1060, 2014. Séminaire Bourbaki. Vol. 2011/2012. Exposés 1059-1065.

[Gol99] W. M. Goldman. Complex hyperbolic geometry. Oxford Mathematical Monographs. The Clarendon Press Oxford University Press, New York, 1999. Oxford Science Publications.

[Gri70] Phillip A. Griffiths. Periods of integrals on algebraic manifolds. III. Some global differential-geometric properties of the period mapping. Inst. Hautes Études Sci. Publ. Math., (38):125-180, 1970.

[Has03] B. Hassett. Moduli spaces of weighted pointed stable curves. Adv. Math., 173(2):316$352,2003$.

[Hel94] S. Helgason. Geometric analysis on symmetric spaces, volume 39 of Mathematical Surveys and Monographs. American Mathematical Society, Providence, RI, 1994.

[Hun00] B. Hunt. Higher-dimensional ball quotients and the invariant quintic. Transform. Groups, 5(2):121-156, 2000.

[Huy05] D. Huybrechts. Complex geometry: An introduction. Universitext. Springer-Verlag, Berlin, 2005.

[KN96] S. Kobayashi and K. Nomizu. Foundations of differential geometry. Vol. II. Wiley Classics Library. John Wiley \& Sons Inc., New York, 1996. Reprint of the 1969 original, A Wiley-Interscience Publication.

[Kon97] M. Kontsevich. Lyapunov exponents and Hodge theory. In The mathematical beauty of physics (Saclay, 1996), volume 24 of Adv. Ser. Math. Phys., pages 318-332. World Sci. Publishing, River Edge, NJ, 1997.

[KZ97] M. Kontsevich. and A. Zorich. Lyapunov exponents and Hodge theory, 1997, arXiv:hepth/9701164.

[Lan02] Serge Lang. Algebra, volume 211 of Graduate Texts in Mathematics. Springer-Verlag, New York, third edition, 2002.

[Lan03] Adrian Langer. Logarithmic orbifold Euler numbers of surfaces with applications. Proc. London Math. Soc. (3), 86(2):358-396, 2003.

[Mar91] G. A. Margulis. Discrete subgroups of semisimple Lie groups, volume 17 of Ergebnisse der Mathematik und ihrer Grenzgebiete (3) [Results in Mathematics and Related Areas (3)]. Springer-Verlag, Berlin, 1991.

[McM13] Curtis T. McMullen. Braid groups and Hodge theory. Math. Ann., 355(3):893-946, 2013.

[McR11] D. B. McReynolds. Arithmetic lattices in $S U(n, 1), 2011$, preprint, available on the author's web page.

[Möl11] M. Möller. Shimura and Teichmüller curves. J. Mod. Dyn., 5(1):1-32, 2011.

[Mos80] G. D. Mostow. On a remarkable class of polyhedra in complex hyperbolic space. Pacific J. Math., 86(1):171-276, 1980. 
[Mos86] G. D. Mostow. Generalized Picard lattices arising from half-integral conditions. Inst. Hautes Études Sci. Publ. Math., (63):91-106, 1986.

[Mos88] G. D. Mostow. On discontinuous action of monodromy groups on the complex $n$-ball. J. Amer. Math. Soc., 1(3):555-586, 1988.

[MVZ12] Martin Möller, Eckart Viehweg, and Kang Zuo. Stability of Hodge bundles and a numerical characterization of Shimura varieties. J. Differential Geom., 92(1):71-151, 2012.

[Par09] J.R. Parker. Complex hyperbolic lattices. In Discrete groups and geometric structures, volume 501 of Contemp. Math., pages 1-42. Amer. Math. Soc., Providence, RI, 2009.

[Pau10] J. Paupert. Unfaithful complex hyperbolic triangle groups. III. Arithmeticity and commensurability. Pacific J. Math., 245(2):359-372, 2010.

[PS03] C. A. M. Peters and J. H. M. Steenbrink. Monodromy of variations of Hodge structure. Acta Appl. Math., 75(1-3):183-194, 2003. Monodromy and differential equations (Moscow, 2001).

[Roy80] H. L. Royden. The Ahlfors-Schwarz lemma in several complex variables. Comment. Math. Helv., 55(4):547-558, 1980.

[Rud87] Walter Rudin. Real and complex analysis. McGraw-Hill Book Co., New York, third edition, 1987.

[Rue79] David Ruelle. Ergodic theory of differentiable dynamical systems. Inst. Hautes Études Sci. Publ. Math., (50):27-58, 1979.

[Sau90] J.K. Sauter, Jr. Isomorphisms among monodromy groups and applications to lattices in PU(1,2). Pacific J. Math., 146(2):331-384, 1990.

[Sch73] W. Schmid. Variation of Hodge structure: the singularities of the period mapping. Invent. Math., 22:211-319, 1973.

[Shi04] H. Shiga. On holomorphic mappings of complex manifolds with ball model. J. Math. Soc. Japan, 56(4):1087-1107, 2004.

[Thu98] W. P. Thurston. Shapes of polyhedra and triangulations of the sphere. In The Epstein birthday schrift, volume 1 of Geom. Topol. Monogr., pages 511-549 (electronic). Geom. Topol. Publ., Coventry, 1998.

[VZ07] E. Viehweg and K. Zuo. Arakelov inequalities and the uniformization of certain rigid Shimura varieties. J. Differential Geom., 77(2):291-352, 2007.

[Wri12] Alex Wright. Schwarz triangle mappings and Teichmüller curves: abelian square-tiled surfaces. J. Mod. Dyn., 6(3):405-426, 2012.

[Yos87] Masaaki Yoshida. Fuchsian differential equations. Aspects of Mathematics, E11. Friedr. Vieweg \& Sohn, Braunschweig, 1987. With special emphasis on the Gauss-Schwarz theory.

[Zor06] A. Zorich. Flat surfaces. In Frontiers in Number Theory, Physics and Geometry. Volume 1: On random matrices, zeta functions and dynamical systems, pages 439-586. Springer-Verlag, Berlin, 2006.

Institut für Mathematik, Goethe-Universität Frankfurt, Robert-Mayer-Str. 6-8, 60325 Frankfurt am Main, Germany

E-mail address: kappes@math.uni-frankfurt.de

E-mail address: moeller@math.uni-frankfurt.de 